This item was submitted to Loughborough's Research Repository by the author.

Items in Figshare are protected by copyright, with all rights reserved, unless otherwise indicated.

\title{
Dispersive and diffusive-dispersive shock waves for non-convex conservation laws
}

PLEASE CITE THE PUBLISHED VERSION

http://dx.doi.org/10.1137/15M1015650

\section{PUBLISHER}

(C) Society for Industrial and Applied Mathematics

\section{VERSION}

AM (Accepted Manuscript)

\section{PUBLISHER STATEMENT}

This work is made available according to the conditions of the Creative Commons Attribution-NonCommercialNoDerivatives 4.0 International (CC BY-NC-ND 4.0) licence. Full details of this licence are available at: https://creativecommons.org/licenses/by-nc-nd/4.0/

\section{LICENCE}

CC BY-NC-ND 4.0

\section{REPOSITORY RECORD}

El, G.A., M.A. Hoefer, and Michael Shearer. 2019. "Dispersive and Diffusive-dispersive Shock Waves for Nonconvex Conservation Laws". figshare. https://hdl.handle.net/2134/21355. 


\title{
DISPERSIVE AND DIFFUSIVE-DISPERSIVE SHOCK WAVES FOR NON-CONVEX CONSERVATION LAWS*
}

\author{
G. A. EL $\dagger$ M. A. HOEFER $\ddagger$ AND M. SHEARER ${ }^{\S}$
}

\begin{abstract}
We consider two physically and mathematically distinct regularization mechanisms of scalar hyperbolic conservation laws. When the flux is convex, the combination of diffusion and dispersion are known to give rise to monotonic and oscillatory traveling waves that approximate shock waves. The zero-diffusion limits of these traveling waves are dynamically expanding dispersive shock waves (DSWs). A richer set of wave solutions can be found when the flux is non-convex. This review compares the structure of solutions of Riemann problems for a conservation law with non-convex, cubic flux regularized by two different mechanisms: 1) dispersion in the modified Korteweg-de Vries $(\mathrm{mKdV})$ equation; and 2$)$ a combination of diffusion and dispersion in the mKdV-Burgers equation. In the first case, the possible dynamics involve two qualitatively different types of DSWs, rarefaction waves (RWs) and kinks (monotonic fronts). In the second case, in addition to RWs, there are traveling wave solutions approximating both classical (Lax) and non-classical (undercompressive) shock waves. Despite the singular nature of the zero-diffusion limit and rather differing analytical approaches employed in the descriptions of dispersive and diffusive-dispersive regularization, the resulting comparison of the two cases reveals a number of striking parallels. In contrast to the case of convex flux, the mKdVB to mKdV mapping is not one-to-one. The mKdV kink solution is identified as an undercompressive DSW. Other prominent features, such as shock-rarefactions, also find their purely dispersive counterparts involving special contact DSWs, which exhibit features analogous to contact discontinuities. This review describes an important link between two major areas of applied mathematics, hyperbolic conservation laws and nonlinear dispersive waves.
\end{abstract}

1. Introduction. Shock waves represent one of the most recognizable features of nonlinear wave systems. In their simplest form, shock waves can be modeled as discontinuous, weak solutions of scalar conservation laws in one space dimension $x$ and time $t$

$$
u_{t}+f(u)_{x}=0 .
$$

When the flux function $f$ is convex, shock admissibility rests upon an entropy condition that uniquely selects the physically relevant weak solution [64]. The existence of entropy presupposes some irreversible dissipative mechanism, e.g., diffusion, in the underlying physical medium. A simple example of a diffusively regularized conservation law is Burgers' equation

$$
u_{t}+\frac{1}{2}\left(u^{2}\right)_{x}=\nu u_{x x}
$$

where $\nu>0$ is a constant representing the diffusion strength.

However, in conservative physical systems whose dominant shock regularizing mechanism is dispersion, there is no corresponding notion of entropy. Discontinuous, weak solutions of (1.1) no longer accurately model wavebreaking dynamics. An alternative theory of dispersive shock waves (DSWs) [45] seeks to examine the asymptotic structure of solutions of dispersive regularizations of (1.1), the simplest example being

* This work was supported by a Royal Society International Exchanges Scheme Grant IE131353. The authors thank G. Esler, E. Ferapontov and A. Kamchatnov for valuable discussions.

${ }^{\dagger}$ Department of Mathematical Sciences, Loughborough University, UK

$\ddagger$ Department of Applied Mathematics, University of Colorado, Boulder, USA; supported by NSF CAREER award DMS-1255422

$\S$ Department of Mathematics, North Carolina State University, Raleigh, NC 27695, USA; supported by NSF grant 
the Korteweg-de Vries (KdV) equation

$$
u_{t}+\frac{1}{2}\left(u^{2}\right)_{x}=\mu u_{x x x}
$$

in which $\mu \neq 0$ is a constant.

The Burgers' (1.2) and KdV (1.3) equations represent two distinctly different ways to regularize the conservation law (1.1) with the convex flux $f(u)=\frac{1}{2} u^{2}$. When the flux in (1.1) is non-convex, new wave features emerge including composite wave solutions and undercompressive shocks that do not satisfy the Lax entropy condition [68]. Many authors have approached the issue of shock wave admissibility when the flux is non-convex by introducing criteria based on mechanics or physics, notably through kinetic relations and nucleation conditions [1, 69]. An additional approach includes regularization of the equation by higher order terms that are dissipative and dispersive, representing more of the physics than is contained in the conservation law (1.1) alone [8,68]. The analysis of shock dynamics in dispersive equations is in many respects quite different from the theory of conservation laws, as exemplified by the famous book of Whitham [97], the pioneering work of Gurevich and Pitaevskii [40] and the series of papers of Lax and Levermore [65]. Much less is known about dispersive shock waves in equations with non-convex flux.

The aim of this paper is to review and compare results of these two regularization approaches for shock wave solutions of (1.1). The comparison is well known for the case of convex flux functions (see, e.g., [46]), but it reveals a number of new nontrivial parallels and contrasts if $f$ is non-convex. This comparison serves two purposes: 1 ) it identifies the dispersive theory as a singular limit of the diffusive-dispersive theory, 2 ) it serves as a bridge between dispersive nonlinear waves and hyperbolic conservation laws, two major areas of applied mathematics. As far as possible, dispersive shock waves are described in the language of hyperbolic conservation laws (characteristics, admissibility criteria, non-classical, undercompressive shocks) and, similarly, diffusive-dispersive shocks are explored in the language of dispersive shock theory (shock polarity and orientation). Most of the results presented here exist in the literature. The purpose of this review is to distill them into a cohesive narrative that contrasts and unifies two areas of applied mathematics.

We focus on the modified KdV-Burgers (mKdVB) equation

$$
u_{t}+\left(u^{3}\right)_{x}=\nu u_{x x}+\mu u_{x x x},
$$

in which $\nu \geq 0$ and $\mu \neq 0$ are parameters. When $\nu=0$, the equation is purely dispersive, and we refer to the equation using the abbreviation $\mathrm{mKdV}$. For $\nu>0$, the equation has both diffusion and dispersion and we refer to it as the mKdVB equation. The modified equation is considered because the combination of non-convex flux with dispersion yields a rich collection of wave solutions. Furthermore, eq. (1.4) can be viewed as a universal model of diffusive-dispersive nonlinear wave dynamics under a certain criticality condition. We demonstrate this by deriving (1.4) from a class of diffusive-dispersive Euler equations.

The applied significance of purely dispersive conservation law regularization is twofold. Firstly, DSW dynamics describe the initial stage of shock development in media with both regularization mechanisms present (subject to an appropriate scale separation of diffusive and dispersive effects). However, the most interesting and rich applications of DSW theory arise in media whose regularization dynamics are dominated by dispersion alone. Prominent examples of such media are Bose-Einstein 
condensates and nonlinear optical media (see, e.g., [46], [84], [95] for relevant experimental and theoretical DSW studies), although conservative nonlinear-dispersive dynamics are also common in water waves and the atmosphere, where DSW-like structures, termed undular bores, have been observed [90], [13].

1.1. Scalar conservation laws. The study of conservation laws goes back to Riemann's analysis of the classic shock tube problem in which a membrane separating gas under different pressures is ruptured, generating a shock wave and a rarefaction wave propagating in opposite directions away from the membrane. This problem is simply represented by the nonlinear system (2.1) of isentropic gas dynamics (see $\S 2)$, in the diffusion and dispersion-free limit $(\nu=0, D=0)$. The study of such hyperbolic systems in one space dimension and time has progressed rapidly since the 1950's, with early work of Lax, Oleinik and others establishing existence of solutions of Riemann problems, shock formation and long-time behavior of solutions, for initial data with small variation. The seminal paper of Glimm [31] injected profound new ideas that were used to prove existence of solutions of initial value problems. As in Glimm's paper, the method now referred to as wave front tracking $[10,15]$ relies on solutions of Riemann problems to approximate weak solutions of hyperbolic systems. Development of the theory in more than one space dimension has been more difficult, but papers of Majda [73, 74] have led the way to further refinements [37].

The theory of scalar conservation laws is much more complete than for systems. For convex fluxes, the theory of initial value problems in one space dimension is classical [63]. The existence and uniqueness results of Kruzhkov [60] in any number of dimensions rely on entropy conditions.

A function $u=u(x, t)$ in $L^{\infty}\left(\mathbb{R} \times \mathbb{R}^{+}\right)$is a weak solution of the initial value problem

$$
u_{t}+f(u)_{x}=0, \quad t>0, x \in \mathbb{R} \quad u(x, 0)=g(x), \quad x \in \mathbb{R},
$$

if for every test function $\phi \in C^{1}\left(\mathbb{R} \times \mathbb{R}^{+}\right)$with compact support, we have

$$
\int_{0}^{\infty} \int_{-\infty}^{\infty}\left(u \phi_{t}+f(u) \phi_{x}\right) d x d t+\int_{-\infty}^{\infty} g(x) \phi(x, 0) d x=0
$$

Here, $\mathbb{R}^{+}=[0, \infty)$, so that the support of $\phi$ can include a portion of the initial line $\mathbb{R} \times\{t=0\}$, and $\phi$ can be non-zero there. When $f$ is smooth and uniformly convex (i.e., there is $\theta>0$ such that $f^{\prime \prime}(u) \geq \theta$ for all $u \in \mathbb{R}$ ), the initial value problem has a unique weak solution whose shocks satisfy the Rankine-Hugoniot conditions (see eq. (3.1) below), but also an entropy condition of Oleinik [79, 80]: there is a constant $C>0$ with the property that

$$
u(x+z, t)-u(x, t) \leq C(1+1 / t) z \text { for almost all } x \in \mathbb{R}, z>0, t>0 .
$$

A readable account of the proof of this result is given in the text of Evans [27]. DiPerna [17] proved asymptotic results for large $t$ for entropy solutions of systems of equations, following earlier analysis of Glimm and Lax [32]. The DiPerna results for scalar equations are proved succinctly in Evans' text.

Another analysis approach is to modify the equations with terms that make initial value problems well posed in suitable function spaces. Typically this regularization will be controlled by a small parameter, $\epsilon$, say; when $\epsilon=0$, the equations revert to the original hyperbolic equations. This is generally a singular limit and the convergence 
is to a function that is less smooth than the approximating solutions. The analysis therefore proceeds in two steps, the first being to show convergence in a suitable topology, the second to show that the limiting function is indeed a weak solution. Additional steps may be needed to show uniqueness of the limit, and to show that the solution satisfies appropriate side conditions such as entropy conditions. Even for scalar equations, the nature of the regularization, and convexity or lack of convexity of the flux, play crucial roles in this approach.

In this paper, we focus on two kinds of regularization, involving either purely dispersive terms, or a combination of dispersive and diffusive terms. For scalar equations with convex flux, these two cases yield strikingly different approximations of shock waves; the differences become even more profound as the flux becomes nonconvex. However, there is also considerable correspondence between the structure of regularized shocks in the purely dispersive case and the structure in the case of mixed dispersive-diffusive regularization.

1.2. DSWs and modulation equations. A DSW manifests as a nonlinear, expanding wavetrain connecting two disparate states of slowly varying (or constant) flow. When viewed locally, i.e., over a small region of space and time, DSWs display periodic, or quasiperiodic structure, forming due to the interplay between nonlinear and dispersive effects. However, over a larger region covering multiple wave oscillations, the DSW wavetrain reveals slow modulation of the wave's parameters (amplitude, frequency, mean etc.), and this modulation itself behaves as a nonlinear hyperbolic wave. This kind of "dispersive-hyperbolic" duality of modulated waves is familiar from linear wave theory, but DSWs prominently display it over a full range of nonlinearity, from a weakly nonlinear regime to solitary waves realized as an integral part of the modulated wave train (see figure 1.1). The striking manifestation of nonlinearity in a DSW is that it is characterized by at least two distinct speeds of propagation, those of its leading and trailing edges. In contrast, energy transport by a linear wave packet is described by a single group velocity. The unsteady dynamics of DSWs have far-reaching physical and mathematical implications, among which are the principal inapplicability of the classical Rankine-Hugoniot relations and inseparability of the macroscopic DSW dynamics from the analysis of its nonlinear oscillatory structure.

The mathematical description of DSWs involves a synthesis of methods from hyperbolic quasi-linear systems, asymptotics, and soliton theory. One of the principal tools is nonlinear wave modulation theory, often referred to as Whitham averaging (Whitham 1965). Whitham theory was first applied to DSWs in the framework of the KdV equation by Gurevich and Pitaevskii [40]. The Gurevich-Pitaevskii approach rests upon the fundamental assumption that the DSW can be asymptotically represented as a slowly modulated periodic traveling wave solution of the original nonlinear dispersive equation, e.g., the KdV equation (1.3), where the spatio-temporal modulation scale (variations of the wave amplitude, wavelength, mean etc.) is assumed to be much greater than the dispersion length, the wavelength scale of the carrier, locally periodic traveling wave (see figure 1.1). This scale separation enables one to effectively split the DSW description problem into two separate tasks of different complexity: the relatively easy problem of the periodic traveling wave solution description and the harder problem of finding an appropriate modulation which provides a match between the modulated wave train (the DSW) with the smooth, slowly varying external flow.

Following Whitham [97], the equations describing slow modulations of periodic nonlinear waves can be obtained by averaging conservation laws of the original dis- 


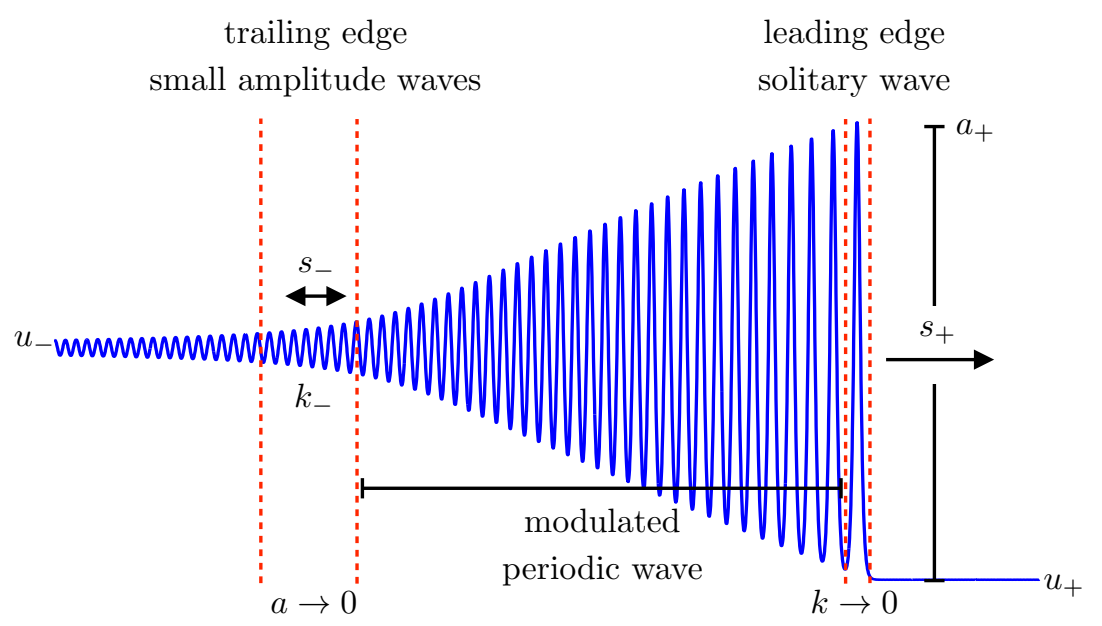

FIG. 1.1. The structure of a DSW with negative dispersion. The amplitude and wave number within the modulated wave are denoted $a(x, t), k(x, t)$, respectively, with limits indicated at the leading and trailing parts of the DSW. The wave speeds $s_{+}, s_{-}$refer respectively to the leading solitary wave, and to the trailing waves. The speed $s_{-}$may be positive or negative.

persive equations over the family of periodic traveling wave solutions. Given an $N$-th order nonlinear evolution equation, implementation of the Whitham method requires the existence of a $N$-parameter family of periodic traveling wave solutions $\phi(\theta ; \mathbf{u})$ with phase $\theta=x-U t$, phase velocity $U(\mathbf{u})$, and parameters $\mathbf{u} \in \mathbb{R}^{N}$. Additionally, the evolution equation must admit at least $N-1$ conserved densities $\mathcal{P}_{i}[\phi]$ and fluxes $\mathcal{Q}_{i}[\phi], i=1, \ldots N-1$ corresponding to the local conservation laws

$$
\frac{\partial}{\partial t} \mathcal{P}_{i}+\frac{\partial}{\partial x} \mathcal{Q}_{i}=0, \quad i=1, \ldots, N-1
$$

We note that in the majority of known, physically relevant dispersive-hydrodynamic systems, the number of parameters characterizing periodic waves is $N=3$ or $N=4$, although systems yielding $N>4$ can arise in some applications (see [24]). For the KdV and $\mathrm{mKdV}$ equations, $N=3$ while for the more general case of Eulerian dispersive hydrodynamics considered in Section $2, N=4$.

Assuming slow evolution of the wave's parameters $\mathbf{u}=\mathbf{u}(x, t)$ on spatio-temporal scales much larger than the wave's wavelength $L(\mathbf{u})$ and period $T(\mathbf{u})=L / U$, the conservation laws are then averaged over a wavelength resulting in the modulation equations

$$
\left(\frac{1}{L} \int_{0}^{L} \mathcal{P}_{i}[\phi(\theta ; \mathbf{u})] d \theta\right)_{t}+\left(\frac{1}{L} \int_{0}^{L} \mathcal{Q}_{i}[\phi(\theta ; \mathbf{u})] d \theta\right)_{x}=0, \quad i=1, \ldots, N-1 .
$$

The $N-1$ averaged local conservation equations (1.7) are completed by the addition of the conservation of waves

$$
k_{t}+\omega_{x}=0,
$$

where $k(\mathbf{u})=2 \pi / L$ and $\omega(\mathbf{u})=k U=2 \pi / T$ are the wavenumber and frequency of the nonlinear traveling wave, respectively. Equation (1.8) represents a consistency 
condition for the application of modulation theory. Note that the system (1.7), (1.8), unlike (1.6), is a system of non-dispersive conservation laws, which, assuming nonvanishing of the relevant Jacobians, can be represented in the standard form of a system of first order quasilinear equations

$$
\mathbf{u}_{t}+\mathrm{A}(\mathbf{u}) \mathbf{u}_{x}=0
$$

The matrix $\mathrm{A}(\mathbf{u})$ encodes information about both the nonlinear and dispersion properties of the original evolution equation. An alternative version of the Whitham method, leading to the same set of modulation equations (1.9), employs an averaged variational principle [98].

In some special but important cases, the system (1.9) can be reduced to diagonal form

$$
\frac{\partial r_{i}}{\partial t}+V_{i}(\mathbf{r}) \frac{\partial r_{i}}{\partial x}=0, \quad i=1, \ldots, N
$$

where $r_{i}\left(u_{1}, \ldots, u_{N}\right)$ are Riemann invariants, and the characteristic speeds $V_{i}(\mathbf{r})$ are eigenvalues of the Jacobian matrix $\mathrm{A}(\mathbf{u}(\mathbf{r}))$. The existence of Riemann invariants dramatically simplifies the modulation equation analysis but is only guaranteed if $N \leq 2$. Remarkably, Whitham found Riemann invariants for the modulation system associated with periodic solutions of the $\mathrm{KdV}$ equation (1.3) where $N=3$ [97]. A similar set of Riemann invariants was found in [18] for the mKdV equation connected with the KdV equation by the Miura transform [76]. Later it was shown that the availability of Riemann invariants for the KdV-Whitham system is intimately linked to the integrable structure of the $\mathrm{KdV}$ equation via the inverse spectral transform [28], [58]. Subsequently, Riemann invariants were found for other modulation systems associated with integrable equations such as the nonlinear Schrödinger equation [29], [81], the Kaup-Boussinesq system [23] and others. We note that the notion of hyperbolicity plays an important role in the analysis of modulation systems: for strictly hyperbolic systems, the mapping $\mathbf{u} \mapsto \mathbf{r}$ is invertible and the characteristic speeds are distinct $V_{1}<V_{2}<\cdots<V_{N}$. We also note a useful representation of the characteristic speeds [39], [62], [51]

$$
V_{i}=\left[1-\frac{L}{\frac{\partial L}{\partial r_{i}}} \frac{\partial}{\partial r_{i}}\right] U(\mathbf{r}),
$$

which follows from the consideration of the wave conservation equation (1.8) as a consequence of the Riemann system (1.10).

The Whitham method can be viewed as a field-theoretic analogue of the KrylovBogoliubov averaging method known in the theory of ordinary differential equations (ODEs). As a matter of fact, the modulation equations (1.9) can also be derived via a rigorous multiple-scale perturbation procedure, an appropriate nonlinear analog of the classical WKB method involving an asymptotic expansion in a formal small parameter characterizing the scale separation. The modulation equations then naturally arise as the solvability conditions, in the space of periodic functions, for the first-order approximation. The leading order approximation yields the periodic solution itself. This approach, however, lacks directness and explicitness of the averaging method and can involve rather cumbersome calculations, especially in non-integrable cases.

It should be stressed that the Whitham system (1.9) describes modulations of nonlinear periodic waves. In the hyperbolic case, the matrix $\mathrm{A}(\mathbf{u})$ generically has 
$N$ distinct real eigenvalues, which are the system's characteristic speeds, sometimes referred to as nonlinear group speeds. In the limit of vanishing amplitude, two of these characteristic speeds merge together and degenerate into the linear group velocity $\omega_{0}^{\prime}(k)$, where $\omega=\omega_{0}(k)$ is the dispersion relation of the linearized original evolution equation. The splitting of the linear group velocity for modulated waves of finite amplitude is one of the most important results of the Whitham theory (see [98] Ch. 15.4). This effect leads to the expanding structure of DSWs.

In the majority of dispersive hydrodynamic problems involving DSW formation, the modulation systems are hyperbolic, ensuring modulational stability of the DSW wavetrain. Hyperbolicity of the modulation system also enables use of the well developed theory of quasilinear systems, such as the method of characteristics, which is not available for the original, dispersive evolution equation.

By applying the Whitham method, the asymptotic description of a DSW effectively reduces to the integration of the quasilinear modulation system (1.9) with certain initial/boundary conditions. These are formulated in terms of a continuous matching of the averaged, mean flow in the DSW region with the smooth external flow at boundaries that are determined along with the solution [40,22]. This nonlinear free boundary problem and its extensions are now commonly known as the GurevichPitaevskii (GP) matching regularization. Importantly, the Whitham equations subject to the GP matching conditions admit a global solution describing the modulations in an expanding DSW. The simplest, yet very important and representative, example of such a solution was obtained in the original paper [40] for a prototypical problem of the dispersive regularization of an initial step, the Riemann problem, for the KdV equation. The DSW modulation solution was found to be a rarefaction wave solution of the Whitham equations. For a more general case of monotone decreasing initial data, the existence of a global modulation solution describing the KdV DSW was proved in [93].

The GP asymptotic DSW construction procedure for the case of the Riemann problem has been extended to non-integrable systems by a priori assuming the existence of a rarefaction wave solution to the Whitham modulation equations [22]. This assumption and the GP matching conditions yield the key, physical features of the DSW including the leading and trailing edge speeds, solitary wave edge amplitude, and small amplitude wave edge modulation wavenumber (see figure 1.1).

Thus, within the context of the Whitham modulation approach, the dispersive regularization of wave breaking in a hydrodynamic system is implemented by the introduction of additional hyperbolic conservation laws (1.7), (1.8) augmented by certain free boundary matching conditions. This procedure is a dispersive counterpart to the classical regularization of viscous shocks, where the inviscid conservation laws are augmented by the Rankine-Hugoniot relations and entropy conditions.

The modulation description of a DSW is formal in the sense that it is based on certain assumptions about the asymptotic structure of the DSW solution and thus, does not resolve the initial DSW development. Its rigorous validation requires the availability of the exact solution, and thus, is only possible for completely integrable systems. The modulation regularization procedure has been rigorously justified for fundamental integrable models such as the KdV and defocusing Nonlinear Schrödinger equations in the framework of the zero dispersion limit of the Inverse Scattering Transform (IST) developed by Lax, Levermore and Venakides [65, 94, 48]. In non-integrable cases, the validation of modulation theory is usually made via comparison with numerical solutions of the original dispersive initial or boundary value problem. We 
note that, even for integrable equations, the direct application of modulation theory to the description of a DSW often has a number of advantages to the complex IST based approaches. Being quasilinear hyperbolic equations, the modulation equations do not have any global restrictions imposed on the solution, e.g., rapid decay as $|x| \rightarrow \infty$ or spatial periodicity. The application of rigorous methods based on the IST usually involve such limitations. Also, the applicability of the Whitham method to non-integrable equations makes the modulation regularization especially attractive for physical applications.

Yet, the modulation approach has its own limitations. In particular, the results of modulation theory have an inherent long-time asymptotic character and do not yield important information about the initial stages of DSW development. A short time asymptotic theory has recently been developed by Dubrovin [19], which seeks to identify the universal oscillatory structure of dispersive hydrodynamic wave breaking. Another limitation to modulation theory is the fundamental assumption about the DSW structure as a locally periodic wave, which can be violated in some regions of the $(x, t)$-plane, in particular near the DSW edges [34, 35]. Other approaches such as rigorous Riemann-Hilbert steepest descent methods [16], [21] as well as the combination of IST with matched asymptotic expansions [3] can yield additional information about the detailed structure of the oscillations. However, for the majority of applications, the results of modulation theory usually provide the sought for qualitative and quantitative information of DSW behavior. The principal feature of the modulation approach is that it provides a unified framework for the analysis of dispersive regularization of nonlinear wave breaking singularities within the well established context of the theory of one-dimensional quasilinear systems.

1.3. Overview of this work. In this paper, we compare solutions of the purely dispersive $\mathrm{mKdV}$ equation to solutions of the diffusive-dispersive mKdVB equation with comparable initial data. We find quite remarkable similarities, but also striking differences in the structure of solutions. It is also of interest that the comparison depends on the sign of the dispersion coefficient $\mu$; for both $\mathrm{mKdV}$ and mKdVB equations, the sign of $\mu$ affects the structure of some solutions. In each case $(\mu>$ $0, \mu<0)$, we classify solutions of initial value problems with jump initial data:

$$
u(x, 0)= \begin{cases}u_{-}, & x<0 \\ u_{+}, & x>0\end{cases}
$$

according to the values of the constants $u_{-}, u_{+}$. We refer to these special initial value problems as Riemann problems. In our comparison of solutions of Riemann problems, we consider times long enough that sustained wave patterns have emerged. These are the long-time asymptotics available explicitly due to the simplicity of the mKdVB equation.

In the purely dispersive case, solutions typically involve DSWs, expanding, slowly modulated wavetrains that provide for a dynamic transition between two different constant values of $u$. As described in the previous section, the asymptotic description of DSWs can be achieved by an averaging procedure that results in a system of quasilinear first-order partial differential equations supplemented with initial/boundary conditions. DSWs resulting from Riemann problems are described by similarity solutions of the modulation equations. In contrast, solutions of the diffusive-dispersive equation involve traveling waves (TWs), solutions of an ordinary differential equation. The TWs approximate shock waves, which are discontinuous weak solutions of the 
conservation law

$$
u_{t}+\left(u^{3}\right)_{x}=0
$$

This equation has characteristic speed $3 u^{2}$, the speed of small disturbances, analogous to the sound speed in a compressible gas. Shock waves travel at the speed $s=$ $u_{+}^{2}+u_{+} u_{-}+u_{-}^{2}$ depending on the left and right limits, given by the Rankine-Hugoniot condition. Consequently, weak shocks (for which $\left|u_{+}-u_{-}\right|$is small), travel at roughly characteristic speed.

One of the most striking comparisons between the two equations is that for $\mu>0$, the $\mathrm{mKdV}$ and $\mathrm{mKdVB}$ equations exhibit special TWs termed undercompressive (in the language of [47]) because the corresponding shock wave

$$
u(x, t)= \begin{cases}u_{-}, & x<s t \\ u_{+}, & x>s t\end{cases}
$$

is subsonic both ahead of and behind the wave:

$$
s<3 u_{ \pm}^{2}
$$

The undercompressive TWs for mKdV have been known in the literature as kinks.

Undercompressive shocks are non-classical in the sense that they do not satisfy the Lax entropy condition. They are called undercompressive by analogy with gas dynamics, in which classical or Lax shocks involve a jump in pressure associated with compression of the gas as a shock wave passes a fixed observer. When a shock fails to satisfy the Lax entropy condition, it is termed non-classical provided it has some relevance for the equation or application under consideration. For scalar equations, the only non-classical shocks are undercompressive in the sense of being either supersonic (see [91]) or subsonic on both sides of the wave. However, for systems of conservation laws, such as a system of magnetohydrodynamics [99], there can be a rich collection of non-classical shocks that have physical relevance, including both undercompressive and overcompressive shocks. See [88] for examples of $2 \times 2$ systems with both undercompressive and overcompressive shock waves.

For $\mu<0$ there are no undercompressive shocks. In this case, the effect of the non-convex flux on the diffusive-dispersive dynamics (1.4) is the occurrence of a double-wave structure known as a shock-rarefaction. In the limit $\nu \rightarrow 0$, the shock wave in the shock-rarefaction represents a single-sided contact discontinuity propagating with characteristic speed [15]. Contact discontinuities find their purely dispersive $(\nu=0)$ counterparts in the form of non-standard, contact DSWs whose modulations are described by special, double-characteristic expansion-fan solutions of the mKdV modulation equations. Such solutions become possible owing to nonstrict hyperbolicity of the mKdV-Whitham modulation system.

The shock wave theory for the mKdVB equation has been constructed in [47] by analyzing traveling wave solutions. Generalizations to different equations and to the Cauchy problem for equation (1.1) using wave front tracking are detailed in the book by LeFloch [68].

There have been several recent developments in the modulation theory for mKdV DSWs. The two types of DSW solutions to the mKdV equation with $\mu<0$ were constructed in [75] using the modulation equations obtained in [18]. These included the usual, KdV-type (convex flux) DSW solutions as well as non-standard sinusoidal undular bores, which we identify here as contact DSWs. The Riemann problem for the 
closely related defocusing complex mKdV equation was considered in [61]. Elements of the DSW theory for the mKdV equation with $\mu>0$ appear in [11] in the context of collisionless plasma physics. A complete classification of the Riemann problem solutions for the Gardner equation, a combined $\mathrm{KdV}-\mathrm{mKdV}$ equation reducible to $\mathrm{mKdV}$, was constructed in the recent paper [54] for both signs of $\mu$. Application of the results for the Gardner equation to the resonant generation of internal undular bores in stratified fluid flows over topography was considered in [55].

Throughout this review, we incorporate direct numerical solutions in order to provide a visual rendering of the underlying mathematics. Here we fully resolve the small scale oscillatory behavior by utilizing a sufficiently small spatio-temporal discretization (see Appendix B). Note that numerical methods producing large scale, weak solutions of diffusive-dispersively regularized non-convex conservation laws that avoid the small scale discretization are an active area of research [70].

In $\S 2$, we execute a derivation of the KdVB and mKdVB equation from a general system of Eulerian equations for isentropic gas dynamics with non-convex pressure law regularized by a combination of viscous dissipation and a generic, third-order dispersion, using nonlinear multiple scale expansions. Similar derivations for particular cases can be found in $[50,2,96,85]$. The main purpose of our derivation is to identify the generic conditions under which the mKdVB model dynamics persist. The condition for the asymptotic mKdVB dynamics to dominate is shown to coincide with the condition for the loss of genuine nonlinearity of the Eulerian system in the non-diffusive, dispersionless limit. The important result of this section is the expression for the dispersion coefficient in the resulting mKdVB equation in terms of the pressure law and linear dispersion relation of the original non-convex Eulerian system. The sign of this coefficient is given by (2.13) and plays the definitive role in determining the qualitative structure of solutions of the Riemann problem for both $\mathrm{mKdV}$ and $\mathrm{mKdVB}$ equations.

Section 3 is devoted to the comparison of classical Lax shocks with their purely dispersive counterparts by reviewing and comparing Riemann problem solutions of the KdVB and KdV equations. The main feature of a DSW, which is a key element of the KdV Riemann problem solution, is its expanding nature characterized by two distinct speeds, in sharp contrast with the steady structure of diffusive-dispersive shocks propagating with a single shock speed and described by TW solutions of the KdVB equation. The notion of a classical DSW is introduced via an analog of the Lax entropy condition formulated in terms of the characteristic speeds of the Whitham modulation system. It is shown that, for the KdV equation, the DSW admissibility condition coincides with the classical shock entropy condition, although the associated Rankine-Hugoniot condition is not applicable to the purely dispersive case due to the unsteady, expanding nature of the DSW.

In $\S 4$, a unified description of the Riemann problem solutions for the mKdVB equation with positive and negative dispersion is presented following the results of [47]. The detailed structure of TW solutions approximating classical and non-classical (undercompressive) shocks is studied, distinguishing between oscillatory and nonoscillatory regimes.

In $\S 5$, the full classification of Riemann problem solutions to the $\mathrm{mKdV}$ equations with positive and negative dispersion terms is constructed in the framework of Whitham modulation theory, following the results of the previous study [54] performed for the Gardner equation. Families of classical and non-classical DSWs are then constructed and the admissibility conditions (5.20) for classical DSWs are in- 
troduced. These conditions include the entropy-analog causality condition and the convexity condition implying a single-wave regularization. In contrast with the $\mathrm{KdV}$ case, the classical DSW admissibility conditions for the $\mathrm{mKdV}$ equation differ from the counterpart conditions obtained for mKdVB classical shocks in the zero-diffusion limit. The distinct cases, where one or both of the classical DSW admissibility conditions fail, fully determine the boundaries in the $u_{-} u_{+}$plane of initial data between regions of solutions with qualitatively different behaviors. Non-strict hyperbolicity of the modulation equations is shown to play the key role in the occurrence and structure of non-classical DSW solutions such as kinks and contact DSWs.

Section 6 is central and provides a bridge between the two theories reviewed in $\S 4$ and $\S 5$. It is devoted to a detailed comparison of the Riemann problem solutions to the $\mathrm{mKdVB}$ equation with the solutions to the $\mathrm{mKdV}$ equation with comparable initial data. This comparison reveals a number of non-trivial parallels and contrasts between the solutions of the two equations. We produce characteristic diagrams for diffusivedispersive and dispersive regularizations. These diagrams, in particular, clarify the complex structure of non-classical mKdV solutions and further elucidate the singular nature of the zero-diffusion limit. One of the manifestations of this singular nature is that the mapping between solutions of the mKdVB and $\mathrm{mKdV}$ Riemann problems is not one-to-one. The ambiguities are resolved in the small-diffusion regime by identifying relevant time scales, including a transient window during which the dispersiondominated dynamics evolve to accommodate the diffusive-dispersive balance. Finally, we show how the two contrasting analytical approaches to the description of diffusivedispersive shocks and DSWs can be reconciled for the regime of small diffusion in the framework of a perturbed modulation theory.

In $\S 7$, we draw conclusions from our study and outline future perspectives. The appendix contains a description of the numerical method used to compute solutions.

2. KdVB and mKdVB equations as weakly nonlinear approximations of diffusive-dispersive Eulerian hydrodynamics. The KdVB and mKdVB equations are prototypical equations, which often arise as unidirectional, weakly nonlinear long-wave approximations of more general diffusive-dispersive equations. For example, the Euler equations for isentropic gas dynamics in one space dimension, regularized by a combination of viscous dissipation and a generic third-order dispersion take the form,

$$
\begin{aligned}
& \rho_{t}+(\rho v)_{x}=0, \\
& v_{t}+v v_{x}+\rho^{-1}[P(\rho)]_{x}=\tilde{\nu} v_{x x}+[D(\rho, v)]_{x} .
\end{aligned}
$$

Here $\rho \geq 0$ is the density and $v$ the velocity of the fluid, although these labels do not necessarily reflect the actual meaning of the corresponding physical entities (e.g. in nonlinear optics). In Eulerian dispersive fluids, the pressure law $P(\rho)$ is an increasing function, $P^{\prime}(\rho)>0$. The parameter $\tilde{\nu}>0$ is the viscosity and $D(\rho, v)$ is a second order differential operator. We mention that the inviscid version of system (2.1) for certain classes of the operators $[D(\rho, v)]_{x}$ is sometimes called the Euler-Korteweg system $[6,7]$.

The linear dispersion relation for system (2.1) has the form

$$
\omega\left(k, \rho_{0}, v_{0}\right)=v_{0} k-\frac{i \tilde{\nu}}{2} k^{2} \pm \omega_{0}\left(k, \rho_{0}\right),
$$

obtained by linearizing (2.1) about the uniform background state $\rho=\rho_{0}, v=v_{0}$ and seeking the solution in the form of a traveling wave proportional to $\exp [i(k x-$ 
$\omega t)$. The \pm signs in $(2.2)$ correspond to the right- and left-propagating wave modes respectively.

The key property of the dispersion relation we need is determined by the longwave expansion of $\omega_{0}\left(k, \rho_{0}\right)$, which we assume generically has the form

$$
\omega_{0}\left(k, \rho_{0}\right)=c_{0} k+\tilde{\mu} k^{3}+o\left(k^{3}\right), \quad 0<k \ll 1, \quad \tilde{\mu} \neq 0,
$$

where $c_{0}=c\left(\rho_{0}\right), c(\rho)=\sqrt{P^{\prime}(\rho)}$ being the long wave speed of sound. The sign $\operatorname{sgn} \tilde{\mu}=\operatorname{sgn}\left\{\omega_{0}^{\prime \prime}\left(k, \rho_{0}\right)\right\}$ is called the sign of dispersion and plays an important role in our consideration. The aim of this section is to outline the derivations of the KdVB and mKdVB equations from the generic system (2.1) of isentropic Euler equations modified by a combination of viscous and dispersive corrections in order to identify the conditions under which the model dynamics, described in the subsequent sections, persist.

We consider uni-directional, weakly nonlinear long-wave approximations of the diffusive-dispersive Euler equations (2.1) by introducing the following multiple scales expansions (see, e.g., [50, 2]).

$$
\begin{aligned}
& \rho=\rho_{0}+\varepsilon \rho_{1}+\varepsilon^{2} \rho_{2}+\ldots, \\
& v=v_{0}+\varepsilon v_{1}+\varepsilon^{2} v_{2}+\ldots,
\end{aligned}
$$

where $\rho_{i}=\rho_{i}(\chi, \tau), v_{i}=v_{i}(\chi, \tau), i \geq 1$, and

$$
\chi=\varepsilon^{p}[x-U t], \quad \tau=\varepsilon^{q} t, \quad 0<\varepsilon \ll 1, \quad p, q>0 .
$$

To keep nonlinearity, dispersion and dissipation in balance we also need to require $\tilde{\nu}=O\left(\varepsilon^{p}\right)$. To this end, along with (2.5) we introduce

$$
\nu=\frac{\tilde{\nu}}{2 \varepsilon^{p}} .
$$

To be definite we consider rightward-propagating waves (the plus sign in (2.2)) whose speed in the long-wave limit is $U=v_{0}+c_{0}$. We also assume, for $i \geq 1$, that $\rho_{i}, v_{i} \rightarrow 0$ as $\chi \rightarrow+\infty$.

\section{KdVB approximation}

The choice $p=1 / 2, q=3 / 2$ yields, assuming the long-wave dispersion behavior (2.3), the KdVB equation for the first order corrections $v_{1}, \rho_{1}$,

$$
\begin{gathered}
\left(v_{1}\right)_{\tau}+\beta v_{1}\left(v_{1}\right)_{\chi}=\nu\left(v_{1}\right)_{\chi \chi}+\tilde{\mu}\left(v_{1}\right)_{\chi \chi \chi}, \\
\text { where } \quad \beta=1+\frac{\rho_{0} P^{\prime \prime}\left(\rho_{0}\right)}{2 P^{\prime}\left(\rho_{0}\right)}=1+\frac{\rho_{0} c^{\prime}\left(\rho_{0}\right)}{c_{0}} .
\end{gathered}
$$

Also, we get

$$
\rho_{1}=\frac{\rho_{0}}{c_{0}} v_{1}
$$


The KdVB approximation (2.7) is valid as long as $|\beta|=O(1), \varepsilon \rightarrow 0$ in (2.8), which is always the case for systems with convex pressure law, $P^{\prime \prime}(\rho)>0$. However, if $P^{\prime \prime}(\rho)<0$ for some interval of $\rho$, then there is the possibility that the nonlinear coefficient $\beta$ could be zero. This occurs if the background density $\rho_{0}$ satisfies the criticality condition

$$
\left.\left(\rho^{2} P^{\prime}(\rho)\right)^{\prime}\right|_{\rho=\rho_{0}}=0, \quad \text { or, equivalently }\left.\quad(\rho c(\rho))^{\prime}\right|_{\rho=\rho_{0}}=0 \text {. }
$$

On the other hand, if $\beta$ remains non-zero, then the hyperbolic limit of equations (2.1) are genuinely nonlinear in the sense of Lax [64] (see, e.g., [15]). Genuine nonlinearity is most succinctly formulated as convexity of the pressure expressed as a function of the specific volume $w=\rho^{-1}: \mathcal{P}^{\prime \prime}(w) \neq 0$ where $\mathcal{P}(w) \equiv P(1 / w)$. Our main interest will be in non-convex systems, for which the genuine nonlinearity condition is violated at exactly one point. In that case, third-order terms become necessary in the expansions (2.4) to account for the higher-order nonlinear term in the resulting equation for $v_{1}$. Then balance is achieved with new scaling parameters $p=1$ and $q=3$ in the transformation (2.5) (see, e.g., [96], [85]). Substituting the new multiple scales expansions (2.4), (2.5) into (2.1) and equating like powers of $\varepsilon$ up to $\varepsilon^{4}$, we obtain the mKdVB equation for the first order corrections,

$$
\left(v_{1}\right)_{\tau}+\gamma\left(v_{1}\right)^{2}\left(v_{1}\right)_{\chi}=\nu\left(v_{1}\right)_{\chi \chi}+\tilde{\mu}\left(v_{1}\right)_{\chi \chi \chi}, \quad \rho_{1}=\frac{\rho_{0}}{c_{0}} v_{1} .
$$

Here,

$$
\chi=\varepsilon\left[x-\left(v_{0}+c_{0}\right) t\right], \quad \tau=\varepsilon^{3} t,
$$

and

$$
\gamma=\frac{\rho_{0}}{4 c_{0}^{3}} \kappa^{\prime \prime \prime}\left(\rho_{0}\right), \quad \text { where } \quad \kappa(\rho)=\rho P(\rho)
$$

Introducing

$$
u=\sqrt{\frac{|\gamma|}{3}} v_{1}, \quad \mu=\tilde{\mu} \operatorname{sgn} \gamma
$$

and replacing $\tau$ by $t, \chi$ by $\operatorname{sgn}(\gamma) x$, we arrive at the standard form (1.4) of the mKdVB equation.

If $\beta$ in $(2.8)$ is not strictly zero but $O(\varepsilon)$, the slightly modified long-wave multiple scales expansions (2.5) with $p=1, q=3$ lead to the mixed, KdV-mKdV-Burgers equation, with both quadratic and cubic nonlinearities present. The zero-diffusion version of this equation is often called the Gardner equation. See [85] and [53] for the multiple scales derivations of the Gardner equation for ion-acoustic waves in collisionless plasmas with negative ions and nonlinear polarization waves in two-component Bose-Einstein condensates respectively.

As mentioned above, the structure of some solutions to the Riemann problem for the $\mathrm{mKdV}$ and $\mathrm{mKdVB}$ equations depends crucially on the sign of the dispersion coefficient $\mu$, which is defined in terms of the key nonlinear and dispersive characteristics of the original Eulerian system (2.1) as

$$
\operatorname{sgn} \mu=\operatorname{sgn}\left[\kappa^{\prime \prime \prime}\left(\rho_{0}\right) \omega_{k k}\left(k, \rho_{0}\right)\right] .
$$


In systems with non-convex dispersion law $\omega_{0}\left(k, \rho_{0}\right)$, in addition to non-convexity of the pressure law, there is the possibility of a sign change in $\mu$ due to a range of values of $k$ involved in the Riemann problem solutions, which can lead to further complexity in their structure. See [72] for the Riemann problem analysis of a dispersive hydrodynamic equation with convex hydrodynamic flux but non-convex dispersion law. However, the possible change of dispersion sign is likely to occur for sufficiently short waves and thus, may not affect the long-wave approximation.

3. Lax shocks and their dispersive counterparts. The shock wave (1.14) is a weak solution of the conservation law (1.1) if it satisfies (1.5), from which the Rankine-Hugoniot condition

$$
-s\left(u_{+}-u_{-}\right)+f\left(u_{+}\right)-f\left(u_{-}\right)=0
$$

can be deduced. Such a solution is a Lax shock if it satisfies the Lax entropy condition relating the characteristic speeds $f^{\prime}\left(u_{ \pm}\right)$to the shock speed $s=\left(f\left(u_{+}\right)-f\left(u_{-}\right)\right) /\left(u_{+}-\right.$ $\left.u_{-}\right)$:

$$
f^{\prime}\left(u_{+}\right)<s<f^{\prime}\left(u_{-}\right)
$$

For the inviscid Burgers equation

$$
u_{t}+\frac{1}{2}\left(u^{2}\right)_{x}=0
$$

Lax shocks are characterized by $s=\frac{1}{2}\left(u_{+}+u_{-}\right)$, with $u_{+}<u_{-}$. In the next subsection, we show that shock wave solutions of (3.3) are Lax shocks if and only if there are traveling wave solutions of the KdVB equation

$$
u_{t}+\frac{1}{2}\left(u^{2}\right)_{x}=\nu u_{x x}+\mu u_{x x x}
$$

with $\nu>0$.

In $\S 3.2$, we describe the purely dispersive counterpart of the Lax shock for $\nu=0$ in (3.4), the KdV equation. The main point we wish to emphasize here is that solutions of the $\mathrm{KdV}$ equation with initial data a Lax shock are quite different in character from those of the KdVB equation, and possess a structure quite distinct from the Rankine-Hugoniot condition or the traveling wave solutions of the KdVB equation (3.4) with $\nu>0$.

3.1. Traveling wave solutions approximating shocks. We wish to consider (3.4) with $\mu \neq 0$. Since the change of variables $x \rightarrow-x, u \rightarrow-u$ leaves the equation unchanged if $\mu$ is replaced by $-\mu$, we can take $\mu<0$ without loss of generality. Then if we seek TW solutions in the form $u(x, t)=\tilde{u}((x-s t) / \sqrt{|\mu|})$ with $\tilde{u}( \pm \infty)=u_{ \pm}$, we see that such solutions approach the shock wave (1.14) as $|\mu|, \nu \rightarrow 0^{+}$and it remains only to demonstrate the existence of the TW solutions.

We find after integrating once that TW solutions satisfy a second order equation

$$
-s\left(u-u_{-}\right)+\frac{1}{2}\left(u^{2}-u_{-}^{2}\right)=\delta u^{\prime}-u^{\prime \prime}, \quad \delta=\nu / \sqrt{|\mu|},
$$

where $\xi=(x-s t) / \sqrt{|\mu|},{ }^{\prime}=d / d \xi$, and we have dropped the tilde from $\tilde{u}$. If $u(\xi)$ is a solution for a fixed $\delta$, then as $\mu \rightarrow 0$, we have $\nu \rightarrow 0$ also. Hence, the traveling wave approaches a shock wave, which is in fact a weak solution of the conservation law (3.3), i.e., (3.4) with $\mu=\nu=0$. Specifically, assuming that $u^{\prime}$ and $u^{\prime \prime}$ approach 


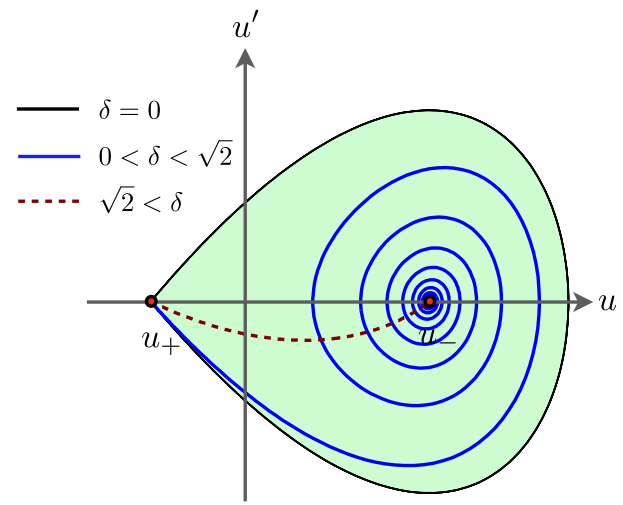

FIG. 3.1. Phase plane depicting KdVB TW solutions connecting $u_{+}<u_{-}$when $\mu<0$. The dashed curve is a monotone TW with strong diffusion. The solid, spiraling curve is an oscillatory $T W$ with weak diffusion. Both are contained inside the homoclinic orbit of the zero diffusion KdV solitary wave solution. The filled region is the union of all KdV periodic orbits spanned across a $D S W$.

zero at $\pm \infty$, the Rankine Hugoniot condition is satisfied: $s=\frac{1}{2}\left(u_{+}+u_{-}\right)$. The second order equation (3.5) can be written as a first order system

$$
u^{\prime}=v, \quad v^{\prime}=\delta v-\frac{1}{2}\left(u^{2}-u_{-}^{2}\right)+s\left(u-u_{-}\right) .
$$

For $\delta>0$, the phase portrait of this system has two equilibria $(u, v)=\left(u_{ \pm}, 0\right)$, one of which is a stable saddle point and the other is an unstable node or spiral, depending on whether the eigenvalues of the linearized system

$$
\lambda_{ \pm}(u)=\frac{1}{2}\left(\delta \pm \sqrt{\delta^{2}+4(u-s)}\right), s=\frac{1}{2}\left(u_{+}+u_{-}\right)
$$

are real $(\delta \geq \sqrt{2 \Delta})$ or complex $(\delta<\sqrt{2 \Delta})$, respectively, where $\Delta=\left|u_{-}-u_{+}\right|$is the jump across the TW (see figure 3.1). An analysis of the phase portrait reveals that the saddle point is connected to the second unstable equilibrium by a heteroclinic orbit, and this corresponds to the TW we seek if and only if $u_{+}<u_{-}$, namely the Lax entropy condition. (Note that for $\mu>0$, the previously unstable node or spiral is stable). The structure of the TW changes from a monotonic to oscillatory profile as $\delta$ is decreased across the critical value $\sqrt{2 \Delta}$. Further analysis shows that for $0<\delta \ll 1$, the leading amplitude of the oscillatory profile is approximately $a=\frac{3}{2} \Delta$ [49]. For $\delta=0$, however, the unstable equilibrium becomes a center, i.e., with imaginary eigenvalues, and a TW connecting $u_{+}$and $u_{-}$no longer exists. Instead, there is a homoclinic orbit connecting the saddle point to itself, and the corresponding TW is a KdV solitary wave. Traveling wave solutions to the KdVB equation were analyzed in $[33,49,9]$.

When $u_{+}>u_{-}$, the inviscid Burgers equation (3.3) has a rarefaction wave solution

$$
u(x, t)= \begin{cases}u_{-}, & x<u_{-} t \\ \frac{x}{t}, & u_{-} t<x<u_{+} t \\ u_{+}, & x>u_{+} t .\end{cases}
$$




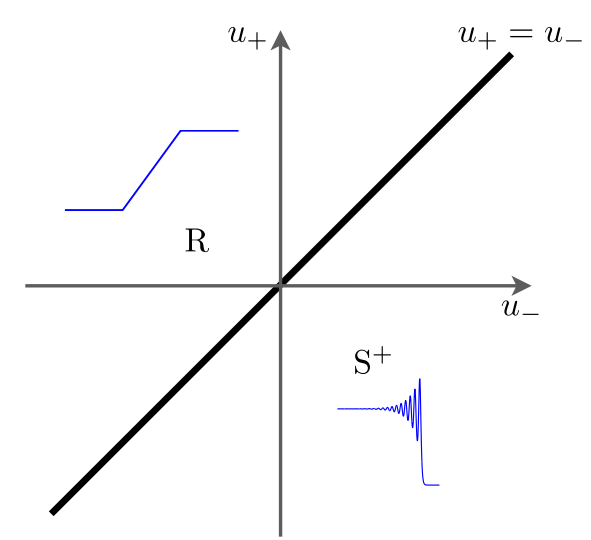

a) $\mu<0,0<\nu<\sqrt{2|\mu|}$

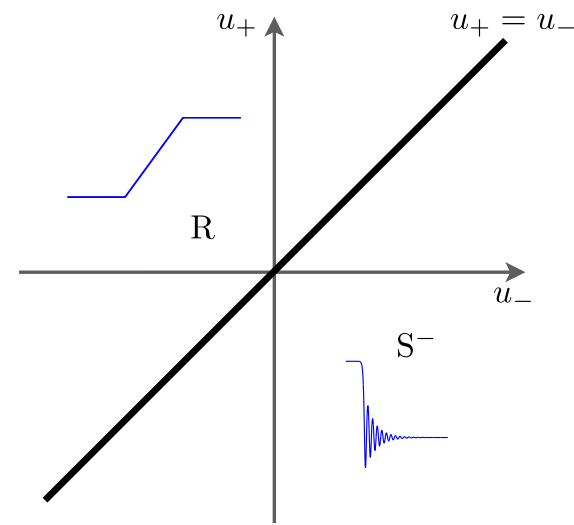

b) $\mu>0,0<\nu<\sqrt{2 \mu}$

FIG. 3.2. Classification of KdVB Riemann problem solutions. (a) Negative dispersion; (b) Positive dispersion.

This solution requires that $u_{+}>u_{-}$. Although rarefaction waves have corners at the edges $x=u_{ \pm} t$, these irregularities are smoothed by the dissipative and dispersive terms, so that apart from this small correction, the rarefaction waves appear for the full KdVB equation.

We can now classify the long-time behavior of solutions of the Riemann problem for (3.4). For $u_{-}>u_{+}$, the solution approaches (as $t \rightarrow \infty$ ) the traveling wave connecting $u_{-}$to $u_{+}$, whereas for $u_{-}<u_{+}$, the solution is approximately a rarefaction wave. This classification is depicted in figure 3.2.

3.2. KdV equation and DSWs. As remarked at the end of $\S 3.1$, when $\nu=0$ in (3.4), there is no traveling wave corresponding to a heteroclinic orbit in the phase portrait, hence the Rankine-Hugoniot condition for a shock wave is not satisfied. Instead, there is a homoclinic orbit connecting the saddle point to itself (a soliton), and there are periodic orbits representing periodic traveling wave solutions of (1.3). In particular, the approximation of a shock by traveling waves is invalid when $\nu=0$. This could lead one to the conclusion that a certain amount of dissipation is necessary to sustain a shock wave transition. Indeed, the classical theory of undular bores by Benjamin and Lighthill [5] and the theory of collisionless shocks in plasma due to Sagdeev [86, 87] and others (see, e.g., [33]) are based on dissipative-dispersive models, essentially reducible to the KdVB equation (3.4). It was not until Gurevich and Pitaevskii's pioneering work [40] that it was realized that there is a qualitatively different type of shock wave that develops in the total absence of dissipation. These conservative shocks are called dispersive shock waves (DSWs) and represent expanding, modulated nonlinear wavetrains connecting the constant states $u_{-}$and $u_{+}$.

The dispersion relation for the $\mathrm{KdV}$ equation (1.3) on the background $u_{0}$ is $\omega\left(k, u_{0}\right)=u_{0} k+\mu k^{3}$. It will be convenient to first consider the case of negative dispersion, $\mu<0$. DSW solutions of the KdV equation are asymptotically described by periodic solutions (cnoidal waves)

$$
u(x, t)=\tilde{u}(\xi)=r_{1}+r_{2}-r_{3}+2\left(r_{3}-r_{1}\right) \operatorname{dn}^{2}\left(\sqrt{\frac{r_{3}-r_{1}}{6}} \xi, m\right)
$$


where $r_{1} \leq r_{2} \leq r_{3}$ are parameters, which remain approximately constant on the wavelength/period scale $\Delta x, \Delta t \sim \sqrt{|\mu|} ; \operatorname{dn}(\xi, m)$ is a Jacobi elliptic function,

$$
\xi=\frac{x-U t+x_{0}}{\sqrt{|\mu|}}, \quad U=\frac{1}{3}\left(r_{1}+r_{2}+r_{3}\right), \quad m=\frac{r_{2}-r_{1}}{r_{3}-r_{1}} .
$$

Here $x_{0} / \sqrt{|\mu|}$ is the initial phase and the modulus $m$ satisfies $0 \leq m \leq 1$. As $m$ is varied from 0 to 1 , the corresponding periodic orbits sweep out a phase plane region enclosed by the solitary wave homoclinic orbit when $m \rightarrow 1^{-}$(see figure 3.1 ). The limit $m \rightarrow 0^{+}$corresponds to the linear, harmonic wave limit. Slow, spatiotemporal modulations $r_{i}(x, t)$ of the function (3.7) occur on a scale substantially larger than $\sqrt{|\mu|}$ and provide the required transition between the two distinct constants $u_{-}$and $u_{+}$so that $m=0$ at one edge of the DSW and $m=1$ at the other edge. Thus the DSW completely fills the phase plane region enclosed by the solitary wave orbit. In figure 3.1, we see that the behavior of the KdVB TW for weak diffusion $0<\delta<\sqrt{2}$ spirals through, but does not fill, this same region.

The modulations are described by a system of three quasilinear, first order (hydrodynamic type) equations derived originally by Whitham [97] by averaging three of the conservation laws of (1.3) over the periodic family (3.7) resulting in

$$
\frac{\partial r_{i}}{\partial t}+V_{i}\left(r_{1}, r_{2}, r_{3}\right) \frac{\partial r_{i}}{\partial x}=0, \quad i=1,2,3,
$$

i.e., $r_{1}, r_{2}, r_{3}$ are Riemann invariants for the modulation equations. The characteristic speeds $V_{1} \leq V_{2} \leq V_{3}$ are found as (see (1.11), (3.8))

$$
V_{i}=U-\frac{L}{3 \frac{\partial L}{\partial r_{i}}}
$$

where

$$
L=2 \mathrm{~K}(m) \sqrt{\frac{6}{r_{3}-r_{1}}},
$$

$\mathrm{K}(m)$ being the complete elliptic integral of the first kind. The quantity $L \sqrt{|\mu|}$ is the wavelength of the periodic wave (3.7). The solution $\mathbf{r}(x, t)$ of the Whitham system (3.9) with appropriate initial or boundary conditions is inserted into the cnoidal wave (3.7) to obtain the asymptotic solution for a DSW. We note that the initial phase $x_{0}$ in (3.8) remains undetermined within Whitham theory so the outlined solution describes the local structure of a modulated wavetrain up to a spatial shift $\sim \sqrt{|\mu|}$ within the traveling wave period.

We now present the explicit expression for $V_{2}\left(r_{1}, r_{2}, r_{3}\right)$, which plays a particularly important role in the DSW theory.

$$
V_{2}\left(r_{1}, r_{2}, r_{3}\right)=\frac{1}{3}\left(r_{1}+r_{2}+r_{3}\right)-\frac{2}{3}\left(r_{2}-r_{1}\right) \frac{(1-m) \mathrm{K}(m)}{\mathrm{E}(m)-(1-m) \mathrm{K}(m)},
$$

where $\mathrm{E}(\mathrm{m})$ is the complete elliptic integral of the second kind. Of particular relevance are two limits of $V_{2}$ corresponding to the harmonic and soliton limits of the traveling wave (3.7). In the harmonic limit, the modulus $m=0$ (i.e. $r_{2}=r_{1}$, see (3.8)) and the characteristic speeds $V_{2}$ and $V_{1}$ merge together

$$
m=0: \quad V_{2}\left(r_{1}, r_{1}, r_{3}\right)=V_{1}\left(r_{1}, r_{1}, r_{3}\right)=-r_{3}+2 r_{1} \equiv V_{-} .
$$


One can also show that in this limit the velocity $V_{3}\left(r_{1}, r_{1}, r_{3}\right)=r_{3}$. Thus, in the harmonic limit the Whitham system (3.9) reduces to a system of two equations, one of which is the inviscid Burgers (dispersionless KdV) equation (3.3) for $r_{3}$.

In the opposite, soliton limit $m=1$ (i.e., $r_{2}=r_{3}$ ), we have a similar degeneracy but now the merged characteristic speeds are $V_{2}$ and $V_{3}$

$$
m=1: \quad V_{2}\left(r_{1}, r_{3}, r_{3}\right)=V_{3}\left(r_{1}, r_{3}, r_{3}\right)=\frac{1}{3}\left(2 r_{3}+r_{1}\right) \equiv V_{+},
$$

while the remaining velocity $V_{1}\left(r_{1}, r_{3}, r_{3}\right)=r_{1}$ yields the inviscid Burgers equation for $r_{1}$.

The described reductions of the KdV-Whitham system (3.9) in the harmonic and soliton limits enable one to construct the solution $\mathbf{r}(x, t)=\left(r_{1}(x, t), r_{2}(x, t), r_{3}(x, t)\right)$ that provides a continuous matching of the period-mean field,

$$
\bar{u}\left(r_{1}, r_{2}, r_{3}\right)=\frac{1}{L} \int_{0}^{L} \tilde{u}(\xi) d \xi=r_{1}+r_{2}-r_{3}+2\left(r_{3}-r_{1}\right) \frac{\mathrm{E}(m)}{\mathrm{K}(m)},
$$

with the distinct constant states $u_{-}, u_{+}$. This results in $\bar{u}\left(r_{1}, r_{1}, r_{3}\right)=r_{3}=u_{-}$at some $x=x_{-}(t)$ (the DSW's trailing, leftmost edge) and $\bar{u}\left(r_{1}, r_{3}, r_{3}\right)=r_{1}=u_{+}$at some $x=x_{+}(t)$ (the leading, rightmost edge). The positions of the DSW edges are free boundaries defined by the merged characteristics $d x_{ \pm} / d t=V_{ \pm}$evaluated for the relevant modulation solution. The outlined matching regularization problem is due to Gurevich and Pitaevskii [40], and its solution $\mathbf{r}(x, t)$ describes slow modulations of the periodic solution (3.7) in a DSW. We note that, while the modulation theory defines the "carrier" cnoidal wave solution (3.7) only up to an arbitrary phase shift $\Delta x \sim \sqrt{|\mu|}$, macroscopic DSW parameters (the amplitude, the wavenumber, the edge speeds, etc.) are fully determined by the solution of the Gurevich-Pitaevskii problem. The computation of the exact phase $x_{0} / \sqrt{|\mu|}$ in the DSW solution would generally require invoking a higher-order approximation, beyond the original leadingorder Whitham theory. Despite this, for some classes of initial data the phase can be explicitly expressed in terms of the Whitham modulation parameters $r_{j}$ [16], [34], [24]. For the case of the Riemann problem considered in this paper, the DSW phase was derived in [3].

The KdV-Whitham system (3.9) is strictly hyperbolic and genuinely nonlinear [71], i.e. for all $\mathbf{r} \in \mathbb{R}^{3}$ one has: (i) $r_{j} \neq r_{k} \Longleftrightarrow V_{j} \neq V_{k}$ (strict hyperbolicity), and (ii) $\partial V_{j} / \partial r_{j} \neq 0, j=1,2,3$ (genuine nonlinearity). Indeed, the merged characteristic speeds (3.12) and (3.13) in the harmonic and soliton limits respectively define a regular characteristic since the order of the modulation system reduces to two in both limits, and thus strict hyperbolicity and genuine nonlinearity of the modulation system are preserved in these limits. As a result, the dispersive regularization of the initial step (1.12) is described by a self-similar simple-wave modulation of (3.7) which is the rarefaction wave solution of the Whitham equations,

$$
\mu<0: \quad r_{1}=u_{+}, \quad r_{3}=u_{-}, \quad V_{2}\left(u_{+}, r_{2}, u_{-}\right)=\frac{x}{t} .
$$

Being a rarefaction fan (see figure 3.3c) solution (3.15) exists for all $t>0$. It is defined in $s_{-} t \leq x \leq s_{+} t$, where $s_{-}$and $s_{+}$are the speeds of the trailing and the leading DSW edges respectively (see figure 3.3). They are found from (3.15) by setting $r_{2}=r_{1}(m=0$ - trailing, harmonic wave edge $)$ and $r_{2}=r_{3}(m=1-$ leading, soliton edge) to obtain (see (3.12), (3.13)) $s_{-}=V_{2}\left(u_{+}, u_{+}, u_{-}\right)=u_{+}-\Delta$ 
and $s_{+}=V_{2}\left(u_{+}, u_{-}, u_{-}\right)=u_{+}+\frac{2}{3} \Delta$, where $\Delta=u_{-}-u_{+}$is the jump across the DSW [40]. It is instructive to note that the speed of the harmonic wave edge coincides with the linear group velocity $\omega_{k}$ evaluated at some $k>0$ while the soliton edge speed is determined by the phase velocity in the limit $k \rightarrow 0$ [22]. The wave number $k=2 \pi / L$ (see (3.11)) in the DSW is a monotone function of $x$, which decreases as the DSW is traversed from the harmonic to solitary wave edge. For $\mu<0$ one has $\partial k / \partial x<0$.

Lax and Levermore [65] showed that this solution of the Whitham equations describes the weak limit solution $u(x, t, \mu)$ of the $\mathrm{KdV}$ equation (1.3) when $\mu \rightarrow 0$, the weak limit being the period-mean (3.14) with $r_{1}, r_{2}, r_{3}$ defined by (3.15). We stress that this weak limit does not coincide with the weak solution of the Riemann problem for the conservation law (3.3), which is the Lax shock (1.14) with $s=\left(u_{-}+u_{+}\right) / 2$. This is due to the oscillations in the DSW having wavelength with scale $\sim \sqrt{|\mu|}$ so the limit as $\mu \rightarrow 0$ is a singular one. Representations of the Riemann problem solutions in the $x$ - $t$ plane for the $\mathrm{KdV}$ equation with $\mu=0$ (the inviscid Burgers equation) and for $\mu \rightarrow 0$ (the Whitham equations) are shown in figure 3.3 .
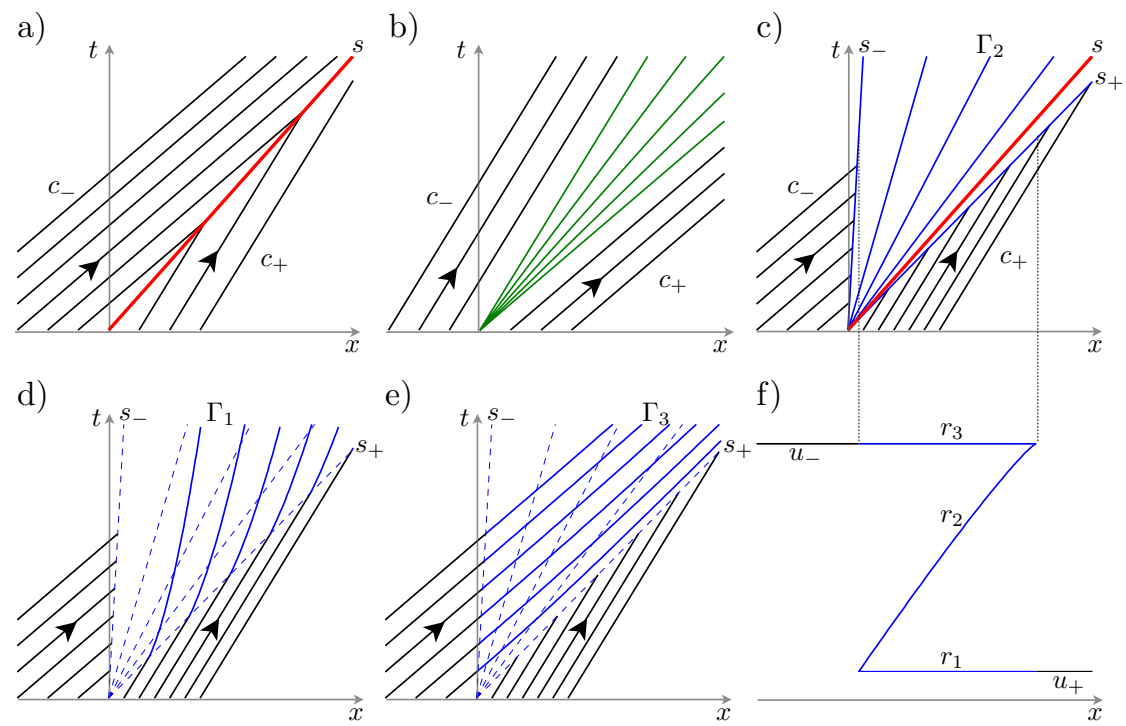

FIG. 3.3. Characteristics for the KdV Riemann problem solutions. (a,b) The inviscid Burgers equation $\mu=0$. (a) $u_{-}>u_{+}$, classical shock; (b) $u_{-}<u_{+}$, rarefaction wave; (c-f) $u_{-}>u_{+}$, $\mu \rightarrow 0$, rarefaction wave of the Whitham equations describing the DSW modulation. (c) The 2characteristic family $\Gamma_{2}$ of the Whitham equations exhibiting the rarefaction fan. (The characteristic corresponding to the classical shock is shown in red.) (d) The 1-characteristics, labeled $\Gamma_{1}$, approach $x=s_{-} t$ as $t \rightarrow \infty$ (2-characteristics are shown as dashed lines for comparison). (e) The 3characteristics, labeled $\Gamma_{3}$, approach $x=s_{+} t$ as $t \rightarrow \infty$. (f) The Whitham Riemann invariants $r_{j}, j=1,2,3$ at a specific time.

The general DSW admissibility conditions for a scalar dispersive equation with convex hyperbolic flux are [22], [24]:

$$
s_{-}<f^{\prime}\left(u_{-}\right), \quad s_{+}>f^{\prime}\left(u_{+}\right), \quad s_{-}<s_{+} .
$$

These conditions are also known as the $D S W$ causality conditions. Similar to the classical Lax entropy conditions (3.2), inequalities (3.16) ensure that the characteristics 
transfer initial data into the DSW region. The generalization of the DSW admissibility conditions (3.16) to bidirectional Eulerian dispersive hydrodynamics can be found in [22], [44]. We shall refer to DSWs satisfying the Lax-type conditions (3.16) as classical DSWs.

For the $\mathrm{KdV}$ equation (1.3), the dispersionless $(\mu=0)$ characteristic speeds are $f^{\prime}\left(u_{-}\right)=u_{-}, f^{\prime}\left(u_{+}\right)=u_{+}$, while speeds of the leading and trailing DSW edges (for $\mu<0$ ) are $s_{+}=u_{+}+\frac{2}{3} \Delta$ and $s_{-}=u_{+}-\Delta$. Thus, in the KdV case, the DSW admissibility conditions (3.16) are equivalent to the entropy condition $u_{+}<u_{-}$ for Lax shocks. However, we stress again that the Rankine-Hugoniot condition $s=$ $\left(u_{-}+u_{+}\right) / 2=u_{+}+\frac{1}{2} \Delta$ is not applicable to a DSW. Moreover, the Lax shock speed satisfies $s_{-}<s<s_{+}$(see the red line in figure 3.3(c)).

As noted for the KdVB equation earlier, the transformation $x \rightarrow-x, u \rightarrow-u$, $\mu \rightarrow-\mu$ leaves the $\mathrm{KdV}$ equation unchanged. Therefore, a reflection of the spatial and amplitude axes of the KdV DSW with negative dispersion, $\mu<0$, is sufficient to describe the DSW with positive dispersion. Explicitly, the traveling wave solution and the modulation equations for the case of positive dispersion are obtained from (3.7) - (3.11) by applying the transformations

$$
\mu<0 \rightarrow \mu>0: \quad u \rightarrow-u, \quad r_{i} \rightarrow-r_{4-i}, \quad V_{i} \rightarrow-V_{4-i}, i=1,2,3 .
$$

We note that under the transformations (3.17), the modulus of the elliptic function transforms as $m \rightarrow 1-m$.

The change $\mu<0 \rightarrow \mu>0$ alters the leading and trailing edge DSW speeds $\left(s_{+}=V_{2}\left(u_{+}, u_{-}, u_{-}\right)=u_{+}+2 \Delta, s_{-}=V_{2}\left(u_{+}, u_{+}, u_{-}\right)=u_{+}+\frac{1}{3} \Delta\right.$ when $\left.\mu>0\right)$ but, perhaps more strikingly, the DSW structure is changed. The local description of the DSW according to (3.7) for $\mu<0$ takes the form of the KdV solitary wave

$$
u=u_{+}+2 \Delta \operatorname{sech}^{2}\left(\sqrt{\frac{\Delta}{6}} \xi\right)
$$

as the parameter $r_{2} \rightarrow r_{3}(m \rightarrow 1)$. Therefore, the leading edge of the DSW exhibits a positive, elevation wave with amplitude $a=2 \Delta$. Whereas for positive dispersion $\mu>$ 0 , the trailing edge of the DSW exhibits a negative, depression solitary wave. Figure 3.4 displays the two cases. The sign of the solitary wave, elevation or depression, defines the polarity $p$ of the DSW. We denote a DSW that contains an elevation $(p=1)$ or depression $(p=-1)$ solitary wave as $\mathrm{DSW}^{+}$or $\mathrm{DSW}^{-}$, respectively.

The position of the solitary wave, at either the leading edge or the trailing edge, defines the orientation $d$ of the DSW. We say that $d=1$ if the solitary wave is at the leading edge, otherwise $d=-1$. The orientation can be found by evaluating $d=-\operatorname{sgn}[\partial k / \partial x]$ at the harmonic edge. This can be understood in very general terms as follows [38]. As already mentioned, the modulation wavenumber $k$ decreases as the DSW is traversed from the harmonic to solitary wave edge. The orientation of the DSW is determined by a well-ordering of the DSW in the vicinity of the harmonic edge. The harmonic edge is at the left (trailing edge) if the group velocity increases with decreasing wavenumber, i.e., $\omega_{k k}<0(\mu<0)$. Conversely, the harmonic edge is at the right (leading edge) if $\omega_{k k}>0(\mu>0)$. In both cases $d=-\operatorname{sgn} \mu$ i.e. the DSW orientation is uniquely defined by the sign of the dispersion.

Thus for the KdV equation, both the DSW polarity and its orientation are determined by the sign of dispersion alone. As we shall see, for the mKdV equation, a change in the DSW polarity (but not the orientation) becomes possible without the 
change of sign of the dispersion. These properties are inherited from the corresponding KdVB traveling wave solutions as indicated by a comparison between figures 3.2 and 3.4 .

If $u_{-}<u_{+}$, the leading order solution of $\mathrm{KdV}$ with $|\mu| \ll 1$ represents a classical rarefaction wave (3.6). In contrast to the Riemann problem solution of the KdVB equation (3.4) with $u_{-}<u_{+}$, one corner (left if $\mu<0$, right if $\mu>0$ ) of the rarefaction wave is smoothed by linear dispersive oscillations whose wavelength $\sim \sqrt{|\mu|}$ and amplitude decays like $t^{-1 / 2}$, while the other corner is exponentially resolved to a constant [67]. The Riemann problem classification for the KdV equation is shown schematically in figure 3.4 .

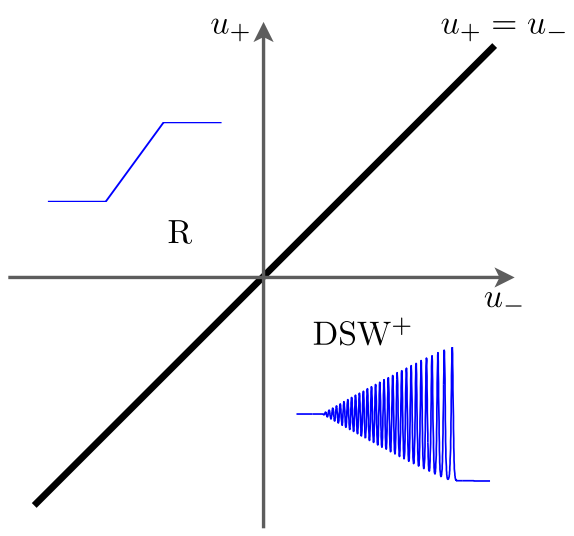

a) $\mu<0, \nu=0$

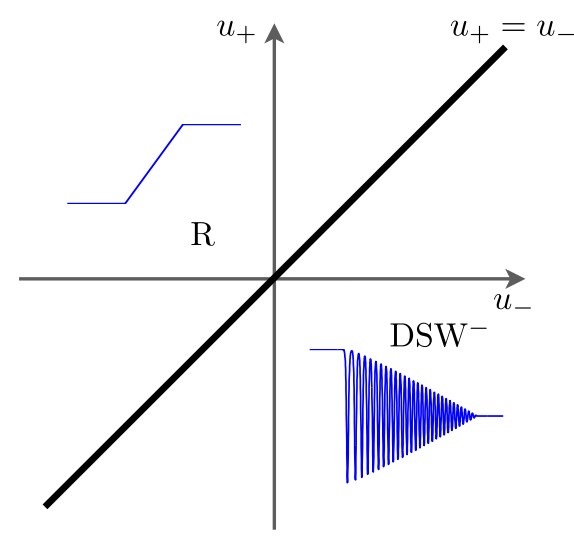

b) $\mu>0, \nu=0$

FIG. 3.4. Classification of the KdV Riemann problem solutions. (a) Negative dispersion; Positive dispersion.

Concluding this section, we summarize the contrasts between KdV DSWs and KdVB traveling shock wave solutions (see figure 3.5) with $0<\nu \ll \sqrt{2 \Delta \mu}$.

1. The most striking contrast between the two structures is that the KdV DSW is an unsteady, expanding structure, and is characterized by two speeds which are defined as the speeds of the leading and trailing edge characteristics of the Whitham modulation equations. The KdVB TW describing the diffusively modified shock is characterized by a single classical shock speed determined by the Rankine-Hugoniot condition.

2. The amplitude of the leading soliton in the KdV DSW is $2 \Delta$ while its counterpart in the shock solution of the KdVB equation with small $\nu>0$ has amplitude $3 \Delta / 2$.

4. mKdVB and non-classical shocks. In this section, we restrict attention to the diffusive-dispersive mKdVB equation (1.4) with $\nu>0$. Our primary interest is to relate traveling waves to shock waves (1.14) satisfying the conservation law

$$
u_{t}+\left(u^{3}\right)_{x}=0 \text {. }
$$

From (3.2) we have that a shock wave solution (1.14) of (4.1) satisfies the Lax entropy condition if

$$
3 u_{+}^{2}<s<3 u_{-}^{2} \text {. }
$$




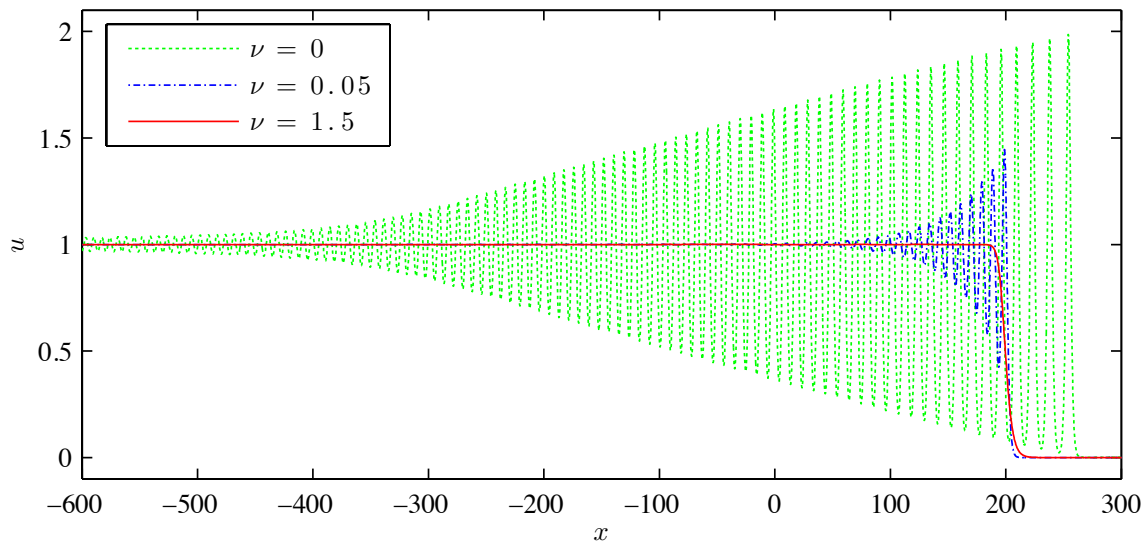

FIG. 3.5. Numerical solutions of the Riemann problem for KdV (dashed, green) and KdVB in the oscillatory (dash-dotted, blue) and monotonic (solid, red) TW regimes with the same step-like initial conditions and $\mu=-1$.

Here,

$$
s=u_{+}^{2}+u_{+} u_{-}+u_{-}^{2}
$$

is the wave speed. Thus, (1.14) is a Lax shock (sometimes termed a classical or compressive shock) if and only if

$$
-\operatorname{sgn}\left(u_{-}\right) \frac{1}{2} u_{-}<\operatorname{sgn}\left(u_{-}\right) u_{+}<\operatorname{sgn}\left(u_{-}\right) u_{-} .
$$

Note the asymmetry in the inequalities resulting from the combination of the Lax entropy condition and the Rankine-Hugoniot relation.

When $u_{+}=-u_{-} / 2$, the shock wave (1.14) satisfies the Lax entropy condition in a limiting sense, since $s=3 u_{+}^{2}$. That is, the shock speed is characteristic on the right. Such a shock is sometimes called a one-sided contact discontinuity.

4.1. Traveling wave solutions. To characterize traveling wave solutions $u=$ $\tilde{u}(x-s t)$ of $(1.4)$ with speed $s$, we impose the far field boundary conditions

$$
\tilde{u}( \pm \infty)=u_{ \pm}, \quad \tilde{u}^{\prime}( \pm \infty)=0, \quad \tilde{u}^{\prime \prime}( \pm \infty)=0 .
$$

Then $\tilde{u}$ satisfies the ODE (dropping the tildes):

$$
-s\left(u-u_{-}\right)+u^{3}-u_{-}^{3}=\nu u^{\prime}+\mu u^{\prime \prime} .
$$

In particular, the boundary condition (4.5) at $+\infty$ yields the Rankine-Hugoniot condition

$$
-s\left(u_{+}-u_{-}\right)+u_{+}^{3}-u_{-}^{3}=0,
$$

giving the wave speed $s$ in (4.3).

The following definition depends on fixing the parameter $\delta=\nu / \sqrt{|\mu|}$ and the sign of $\mu$. A shock wave (1.14) is termed admissible if there is a traveling wave solution of (1.4) satisfying the far-field conditions (4.5). Consequently, the shock wave is 
admissible if and only if the parameters $s, u_{+}, u_{-}$are related by (4.3) and there is a solution of the ODE (4.6) satisfying $u( \pm \infty)=u_{ \pm}$. We note that the ODE is unchanged under the transformation $u \rightarrow-u, u_{-} \rightarrow-u_{-}$. Consequently, if (1.14) is an admissible shock with speed $s$, then so is the shock with $u_{ \pm}$replaced by $-u_{ \pm}$, with the same speed $s$.

Just as for the KdVB equation, we have a second order ODE that describes traveling waves $u(\xi), \xi=(x-s t) / \sqrt{|\mu|}$, except that for the MKdVB equation, the sign of $\mu$ is significant:

$$
\operatorname{sgn}(\mu) u^{\prime \prime}+\delta u^{\prime}=u^{3}-u_{-}^{3}-s\left(u-u_{-}\right), \delta=\nu / \sqrt{|\mu|} .
$$

The corresponding first order system is

$$
u^{\prime}=v, \quad \operatorname{sgn}(\mu) v^{\prime}=-\delta v+\left(u^{3}-u_{-}^{3}\right)-s\left(u-u_{-}\right) .
$$

For $\delta>0$, the phase portrait of this system has between one and three equilibria including $(u, v)=\left(u_{ \pm}, 0\right)$, where $u_{+}$satisfies (4.7). We are interested only in cases for which there are three equilibria, two of which may be a double root of (4.7). The nature of the equilibria depends on the eigenvalues

$$
\lambda_{ \pm}(u)=\frac{1}{2}\left(-(\operatorname{sgn} \mu) \delta \pm \sqrt{\delta^{2}+(\operatorname{sgn} \mu) 4\left(3 u^{2}-s\right)}\right), s=u^{2}+u u_{-}+u_{-}^{2} .
$$

Consider the case of three equilibria with $u=u_{ \pm}, u_{0}$ satisfying $u_{+}<u_{0}<u_{-}$. Then shocks from $u_{-}$or $u_{+}$(with $x<s t$ ) to $u_{0}$ (with $x>s t$ ) satisfy the Lax entropy condition, since $3 u_{0}^{2}<s<3 u_{ \pm}^{2}$.

In terms of the equilibria, the eigenvalues (4.8) imply that if $\mu<0$, then $(u, v)=$ $\left(u_{ \pm}, 0\right)$ are unstable nodes or spiral nodes (if $\delta^{2}+4\left(3 u_{0}^{2}-s\right) \geq 0$ or $\delta^{2}+4\left(3 u_{0}^{2}-s\right)<0$, respectively), and $\left(u_{0}, 0\right)$ is a saddle point. By tracing the stable manifold of $\left(u_{0}, 0\right)$ backwards in time, we can establish that there is a trajectory from each of $\left(u_{ \pm}, 0\right)$ to $\left(u_{0}, 0\right)$. Consequently, for $\mu<0$, all Lax shocks are admissible and there are no other admissible shocks. An example phase portrait is shown in figure 4.1(a).

On the other hand, if $\mu>0$, then $(u, v)=\left(u_{ \pm}, 0\right)$ are saddle points, and $\left(u_{0}, 0\right)$ is a stable equilibrium, either a node or a spiral. The saddle points at $\left(u_{ \pm}, 0\right)$ may or may not be connected to $\left(u_{0}, 0\right)$, and can be connected to each other. See figure 4.1(b) for a phase portrait when both $u_{ \pm}$are connected to $u_{0}$, corresponding to admissible Lax shocks. Figure 4.1(c) is a phase portrait showing a connection for a Lax shock but also an orbit connecting $u_{-}$to $u_{+}$. In this case, there is no connection from $u_{-}$ to $u_{0}$, even though these constants define a Lax shock. It is proved in [47] that an orbit joining saddle points $u_{ \pm}$exists if and only if it lies on an invariant parabola $v= \pm \frac{1}{\sqrt{2}}\left(u-u_{-}\right)\left(u-u_{+}\right)$; an explicit calculation shows that this occurs precisely for

$$
\left|u_{-}\right|>\frac{2 \sqrt{2}}{3} \delta, \quad u_{+}=-u_{-}+\operatorname{sgn}\left(u_{-}\right) \frac{\sqrt{2}}{3} \delta,
$$

for which $s=u_{-}^{2}-\frac{\sqrt{2}}{3} \delta\left|u_{-}\right|+\frac{2}{9} \delta^{2}$, and $u_{0}=-\operatorname{sgn}\left(u_{-}\right) \frac{\sqrt{2}}{3} \delta$. These values help determine which Lax shocks are admissible for $\mu>0$, and which are not. The dynamics on the invariant parabola correspond to the traveling wave

$$
u(\xi)=\frac{1}{2}\left\{u_{-}+u_{+}-\left|u_{-}-u_{+}\right| \tanh (A \xi)\right\}, A=\frac{1}{2 \sqrt{2}}\left(u_{-}-u_{+}\right), \xi=(x-s t) / \sqrt{\mu} .
$$




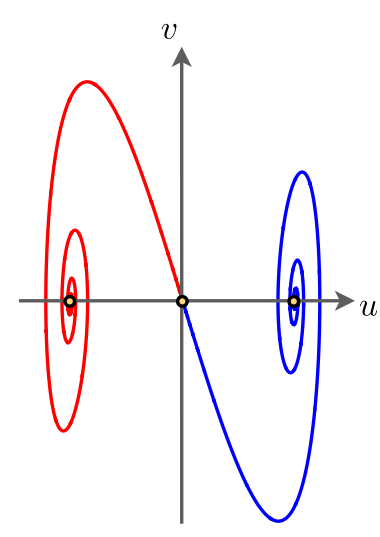

a) $\mu<0$

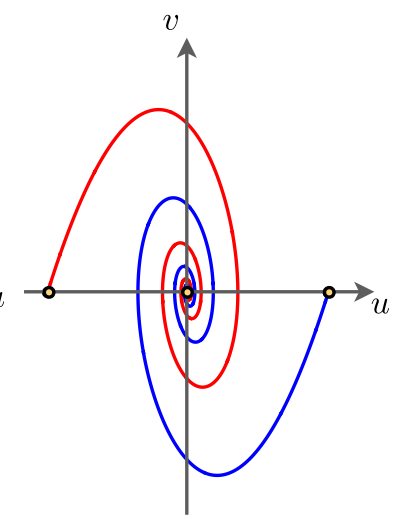

b) $\mu>0,-\frac{\sqrt{2}}{3} \delta<u_{+}<u_{-}$

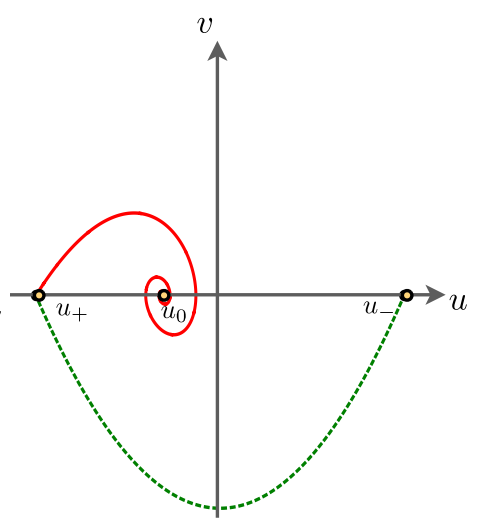

c) $\mu>0, u_{+}=-u_{-}+\frac{\sqrt{2}}{3} \delta$

FIG. 4.1. Representative phase portraits for traveling waves of the mKdVB equation. The solid curves correspond to Lax shocks connecting $u_{ \pm}$to $u_{0}$. The dashed curve in (c) represents an undercompressive shock connecting $u_{-}$to $u_{+}$.

To summarize, for $\left|u_{-}\right|>\frac{2 \sqrt{2}}{3} \delta$, a shock wave (1.14) from $u_{-}$to $u_{+}$is admissible if either (i) $u_{+}$is between $-\operatorname{sgn}\left(u_{-}\right) \frac{\sqrt{2}}{3} \delta$ and $u_{-}$or (ii) $u_{+}=-u_{-}+\operatorname{sgn}\left(u_{-}\right) \frac{\sqrt{2}}{3} \delta$. In case (i), the shock satisfies the Lax entropy condition $3 u_{+}^{2}<s<3 u_{-}^{2}$, and in case (ii) the shock is undercompressive, in the sense that it has the property (1.15) discussed in the introduction. In case (ii), other Lax shocks (for which $u_{+}$lies between $-\frac{1}{2} u_{-}$and $\left.-\operatorname{sgn}\left(u_{-}\right) \frac{\sqrt{2}}{3} \delta\right)$ are not admissible. In the case $\left|u_{-}\right| \leq \frac{2 \sqrt{2}}{3} \delta$, there is no undercompressive shock (for any $u_{+}$) and all Lax shocks (for which $u_{+}$lies between $u_{-}$and $\left.-\frac{1}{2} u_{-}\right)$are admissible.

When $u_{+}=-u_{-} / 2$, the shock wave (1.14) is a one-sided contact discontinuity. It is admissible if either $\mu<0$, or $\mu>0$ and $\left|u_{-}\right| \leq \frac{2 \sqrt{2}}{3} \delta$, the latter inequality arising from the existence of the saddle-saddle connection of Figure 4.1(c).

4.2. The Riemann problem. To complete the description of solutions of the Riemann problem, we need to utilize not only constant solutions and admissible shocks, but rarefaction waves. These are continuous self-similar solutions of (4.1) that join constants $u_{ \pm}$, and are given by the explicit formula

$$
u(x, t)= \begin{cases}u_{-}, & x<3 u_{-}^{2} t \\ \pm \sqrt{\frac{x}{3 t}}, & 3 u_{-}^{2} t<x<3 u_{+}^{2} t \\ u_{+}, & x>3 u_{+}^{2} t .\end{cases}
$$

Rarefaction waves therefore propagate to the right, and $u(x, t)$ has a single sign throughout the wave. Consequently, $\left|u_{+}\right|>\left|u_{-}\right| \geq 0$. Note that the graph of $u=u(x, t)$ as a function of $x$ for fixed $t>0$ is continuous, and smooth apart from corners where the parabola meets the constants $u=u_{ \pm}$. Using this information about shock waves and rarefaction waves, we can construct solutions of the Riemann problem for any $u_{+}, u_{-}$using only constant solutions, admissible shock waves (both classical and undercompressive), and rarefaction waves. The classification diagram for these solutions is presented in figure 4.2. The construction labeled $S^{j} R$, with $j= \pm, 0$, 


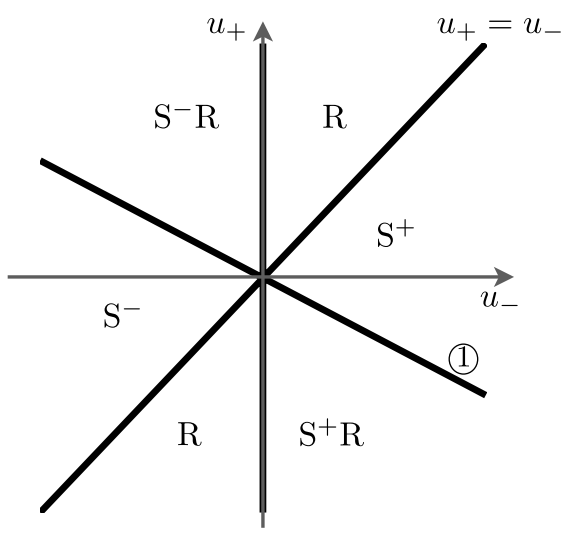

a) $\mu<0, \delta=\nu / \sqrt{|\mu|}>0$

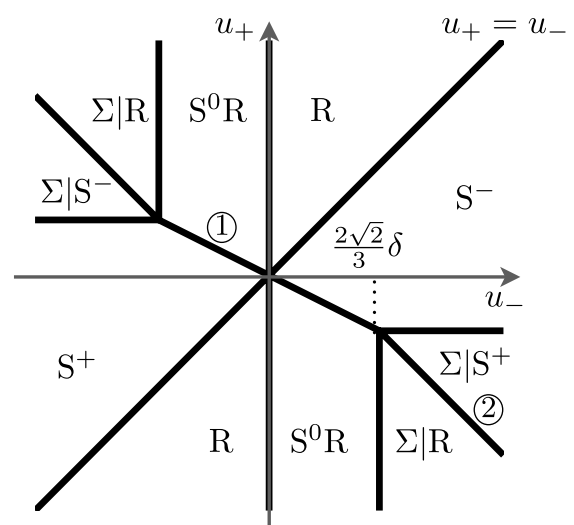

b) $\mu>0, \delta=\nu / \sqrt{\mu}>0$

FIG. 4.2. Solutions of the Riemann problem for the $m K d V B$ equation with $\delta=\nu / \sqrt{|\mu|}>0$. Line (1): $u_{+}=-u_{-} / 2$. Line (2): $u_{+}=-u_{-}+\sqrt{2} \delta / 3$.

indicates a shock-rarefaction. This is a composite wave in which a shock from $u_{-}$ to $-u_{-} / 2$ has speed $s=3 u_{-}^{2} / 2$ that is characteristic on the right, at $u=-u_{-} / 2$. Consequently, to reach $u_{+}$, beyond $-u_{-} / 2$ (meaning $u_{+}<-u_{-} / 2$ when $u_{-}>0$ for example), a faster rarefaction wave can be attached to the shock wave, yielding the single wave structure in which the rarefaction wave has a discontinuity at it's trailing edge. The discontinuity is sometimes called a one-sided contact.

In figure $4.2(\mathrm{a})$, in which $\mu<0$, we use the fact that there are no undercompressive shocks and all Lax shocks are admissible. In figure 4.2(b), we show the structure of solutions when $\mu>0$. The classification is more complicated, in that there are new regions in the diagram, in which the Riemann problem solution has two waves traveling with different speeds, separated by a widening interval in which $u$ is constant. This structure is indicated in the figure with a vertical line between the wave letters. One wave is an undercompressive wave, indicated with the letter $\Sigma$, the other being a shock $\mathrm{S}$ or rarefaction R. Note that figures $4.2(\mathrm{a}, \mathrm{b})$ inherit the symmetry $u_{+} \rightarrow-u_{+}$, $u_{-} \rightarrow-u_{-}$from the governing mKdVB equation.

Strictly speaking, figure 4.2 shows solutions of the Riemann problem for the conservation law (1.13), using information about traveling waves with $0<\delta<\infty$ to distinguish those shocks that are admissible. But the conservation law can be regarded as the expressing the leading order long-time behavior of solutions of the Riemann problem for the MKdVB equation as $\mu$ and $\nu$ approach zero with $\delta>0$ held constant. In figure 6.6, we show numerical solutions of the Riemann problem in representative cases of each of the regions shown in figure 4.2. In that figure, shocks are replaced by smooth traveling waves, and rarefaction waves, though not precisely self-similar, are clearly visible.

It remains to explain the superscripts, \pm 0 associated with shock waves. These indicate whether there may be oscillations $( \pm)$ or not $(0)$. As explained below in the context of purely dispersive shocks, $S^{+}$indicates that the traveling wave terminates exponentially at an equilibrium, with an adjacent maximum. Similarly, $S^{-}$indicates that the largest oscillation involves a minimum before the traveling wave terminates exponentially at the equilibrium. 
To explain these structures, we refer to the ODE (4.6). We can write the equation as a first order autonomous system

$$
u^{\prime}=v, \quad \mu v^{\prime}=-\nu v+f\left(u ; u_{-}, s\right), \quad f\left(u ; u_{-}, s\right)=u^{3}-u_{-}^{3}-s\left(u-u_{-}\right) .
$$

See figure 4.1 for representative phase portraits. Traveling waves corresponding to Lax shocks are given by trajectories for this system between a saddle point equilibrium and a second equilibrium, either a node or spiral. Oscillations are associated with a spiral equilibrium, at which the two eigenvalues are complex conjugates. Since either $u_{+}$or $u_{-}$can be the saddle point, we examine eigenvalues for both, indicating whether there are oscillations behind or ahead of the wave. We find that the sign of $\mu$ is also significant.

Intuitively, we expect that for small $\nu>0$, there will be oscillations, since this is similar to underdamping of oscillations. For large $\nu$ however, there should be no oscillations, corresponding to overdamping. We verify this distinction with a quantitative prediction of the boundary between the presence of oscillations in the traveling wave, and the absence of oscillations. The prediction is then observed in numerical simulations (see $\S 6$ ).

If we linearize system (4.12) about an equilibrium $u$, we find eigenvalues $\lambda$ given by the formula

$$
\lambda=\frac{1}{2}\left\{-\frac{\nu}{\mu} \pm \sqrt{\left(\frac{\nu}{\mu}\right)^{2}+4 \frac{f_{u}}{\mu}}\right\} .
$$

Consequently, the boundary between real and complex eigenvalues is $\frac{\nu^{2}}{\mu}+4 f_{u}=$ 0 . Using the definition (4.12) of $f\left(u, u_{-}, s\right)$ and the formula (4.3) for $s$, we obtain hyperbolas in the $u_{-}, u_{+}$plane for each of $u=u_{-}, u=u_{+}$:

$$
\begin{array}{ll}
u=u_{-}: & \left(2 u_{-}+u_{+}\right)\left(u_{-}-u_{+}\right)=-\frac{\nu^{2}}{4 \mu} \\
u=u_{+}: & \left(2 u_{+}+u_{-}\right)\left(u_{+}-u_{-}\right)=-\frac{\nu^{2}}{4 \mu} .
\end{array}
$$

These are represented in figure 4.3. The hyperbolas are oriented differently depending on the sign of $\mu$. Also in the figure, we indicate the sectors corresponding to Lax shocks.

There are several conclusions to be drawn from these figures.

1. The dashed curves lie outside the sectors representing Lax shocks. Therefore, when $\mu>0$, Lax shocks are non-oscillatory at $u_{-}$and for $\mu<0$, they are non-oscillatory at $u_{+}$.

2. For small enough $\nu$, Lax shocks are oscillatory at $u_{-}$if $\mu>0$, and they are oscillatory at $u_{+}$if $\mu<0$.

Summarizing, we conclude that for $\nu \geq 0$ small enough, Lax shocks are oscillatory on the left (at $u_{-}$) and non-oscillatory on the right (at $u_{+}$) if $\mu>0$, and the reverse is true if $\mu<0$. Therefore we can identify the shock orientation $d=-\operatorname{sgn}(\mu)$, analogous to the orientation of a DSW (recall $\S 3.2$ ).

However, there is a significant difference between the location of the solid line hyperbolas in the two figures. In figure 4.3(a), the hyperbola crosses the line $u_{+}=$ $-u_{-} / 2$, representing the shock in a shock-rarefaction wave, whereas in figure 4.3(b), the hyperbola is contained within the sector representing Lax shocks. Correspondingly, for fixed $\mu>0$, and $\nu>0$, rarefaction-shocks have an oscillation on the left, 
whereas for $\mu<0$, rarefaction-shocks are monotonic. In both cases, weak shocks (those for which $\left|u_{+}-u_{-}\right|$is small) are monotonic.

The superscripts in figure 4.2 are related to the appearance of oscillations for small $\delta>0$. For $\mu<0$, there are oscillations on the left of the wave, so that for $u_{+}<u_{-}$, the final oscillation has a maximum, indicating an $S^{+}$wave, whereas in an $S^{-}$shock, we have $u+>u_{-}$, for which the final oscillation necessarily has a minimum. Note that in a solution designated $\Sigma \mid S^{+}$, the shock is actually properly associated with the $S^{+}$ region with $u_{+}>u_{-}$. A similar remark applies to the $\Sigma \mid S^{-}$solutions. The $S^{0}$ shocks have monotonic traveling waves, in agreement with the remarks above concerning figure $4.3(\mathrm{~b})$.

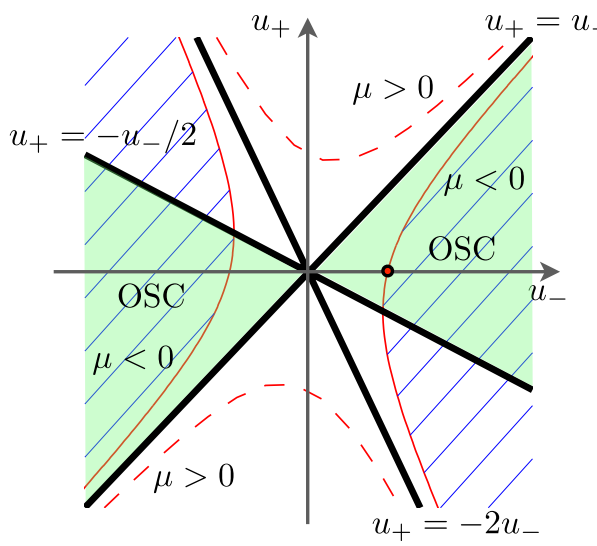

a) $u=u_{-}$

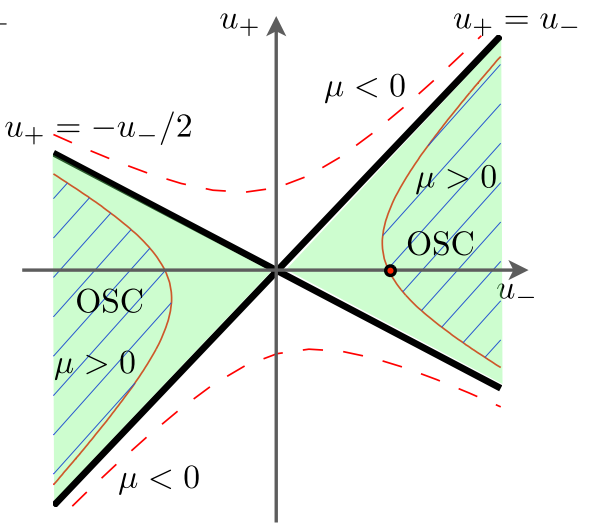

b) $u=u_{+}$

FIG. 4.3. The hyperbolas delineating the boundary between oscillatory and non-oscillatory traveling waves. Lax shocks correspond to the shaded areas. (a) Region OSC where oscillations occur at $u_{-}$, when $\mu<0$. (b) Region OSC where oscillations occur at $u_{+}$when $\mu>0$. The dot on the $u_{-}$-axis is at $u_{-}=\nu / 2 \sqrt{2|\mu|}$ in (a), and at $u_{-}=\nu / 2 \sqrt{\mu}$ in (b).

We have shown that there is an important distinction between Riemann problem solutions to the mKdVB equation depending on dispersion. When $\mu<0$, the effect of the dispersive term is the introduction of oscillations when $\delta=\nu / \sqrt{|\mu|}$ is sufficiently small. Otherwise, the solutions are classical in the sense that shocks satisfy the Lax entropy condition. The classification diagram in figure 4.2(a) is independent of $\delta>0$. While the $\mu>0$ case also yields oscillatory Lax shocks, this regime supports non-classical, undercompressive shocks that do not satisfy the Lax entropy condition. Here, dispersion plays a more significant role, which manifests as a $\delta$ dependent classification diagram (figure 4.2(b)). Note that the limit $\delta \rightarrow \infty$ (i.e., $\mu \rightarrow 0$, the purely diffusive case) of figure 4.2 (b) leads to the same Riemann problem classification as in figure 4.2(a), i.e., the Riemann problem is independent of $\nu$ when $\mu \rightarrow 0$.

5. Riemann problem for the mKdV equation. In this section, we present the classification of Riemann problem solutions for the mKdV equation, i.e., (1.4) with $\nu=0$. Similar to the KdVB equation discussed in $\S 3$, the transition to the inviscid case $\nu=0$ in the mKdVB equation is singular in the sense that although Lax shocks may have corresponding traveling waves for $\nu>0$, they are not present for $\nu=0$. Instead, the Lax shocks are replaced by DSW solutions. In contrast to the $\mathrm{KdVB} / \mathrm{KdV}$ 
case, non-convexity of the hyperbolic flux in the case of the $\mathrm{mKdVB} / \mathrm{mKdV}$ equations results in a much richer variety of solutions, which makes establishing the parallels between the diffusive-dispersive and purely dispersive cases rather non-trivial. One of the pertinent questions arising in this connection is: what is a conservative, purely dispersive counterpart of an undercompressive shock in the $\mu>0$ case? A second question concerns the identification of dispersive counterparts of composite wave structures such as shock-rarefactions, occurring as solutions of the mKdVB equation for both $\mu>0$ and $\mu<0$.

The full classification of Riemann problem solutions for the Gardner equation, the extended version of the $\mathrm{mKdV}$ equation containing both quadratic and cubic nonlinearities, was constructed in [54]. In that paper, the Gardner equation was taken in the standard form $w_{t^{\prime}}+6 w w_{x^{\prime}}-6 \alpha w^{2} w_{x^{\prime}}+w_{x^{\prime} x^{\prime} x^{\prime}}=0$, which can be reduced to the $\mathrm{mKdV}$ equation in the form (1.4) with $\nu=0, \mu=\operatorname{sgn} \alpha$ by the change of variables $u=\sqrt{2|\alpha|}[w-1 /(2 \alpha)], t=t^{\prime}, x=-\left[x^{\prime}-3 t^{\prime} /(2 \alpha)\right] \operatorname{sgn} \alpha$. Here we adapt the classifications of [54] for both signs of $\alpha$ to the pure mKdV case (with the corresponding signs of $\mu$ ) to enable detailed comparison with the counterpart mKdVB classifications outlined in $\S 4$.

5.1. mKdV equation: DSW theory. To produce the classification of asymptotic solutions to the Riemann problem for the mKdV equation, one needs first to construct elementary building blocks such as DSWs and rarefaction waves, and identify their admissibility conditions. For this, we first describe $\mathrm{mKdV}$ periodic solutions and their limiting configurations, and then develop modulation theory for mKdV DSWs. Unlike the well-established theory of diffusive-dispersive shocks for the mKdVB equation, the counterpart $\mathrm{mKdV}$ theory is not directly available from a single source so we present here a unified account of previous results along with further development in the context of the theory of hyperbolic conservation laws.

5.1.1. Traveling waves. Traveling wave solutions of the $\mathrm{mKdV}$ equation are available from a number of papers (see, e.g., $[56,75]$ ) and can also be obtained as reductions of corresponding solutions to the Gardner equation [54].

Traveling wave solutions to the $\mathrm{mKdV}$ equation are sought in the form $u=$ $\tilde{u}(x-U t)$, with speed $U$. Note that we have omitted the initial phase in the TW ansatz here as this does not contribute to the modulation solution of the Riemann problem (see Section 3.2 for the discussion of the DSW phase for the KdV case).

After integrating twice, one arrives at the ODE (dropping the tildes)

$$
u_{\eta}^{2}=\operatorname{sgn} \mu\left(u-u_{1}\right)\left(u-u_{2}\right)\left(u-u_{3}\right)\left(u-u_{4}\right) \equiv Q(u),
$$

where $\eta=\frac{x-U t}{\sqrt{2|\mu|}}$ and the roots of the polynomial $Q(u)$ satisfy the constraint

$$
\sum_{i=1}^{4} u_{i}=0 .
$$

While only three roots $u_{j}$ are independent, it is still convenient to keep all four $u_{j}$ 's in the subsequent formulae to preserve symmetry of the expressions. In the modulationally stable case of our interest, all $u_{j}$ 's are real. We assume the ordering

$$
u_{1} \leq u_{2} \leq u_{3} \leq u_{4} .
$$

The phase velocity $U$ is expressed in terms of the $u_{j}$ 's as

$$
U=-\frac{1}{2}\left(u_{1} u_{2}+u_{1} u_{3}+u_{1} u_{4}+u_{2} u_{3}+u_{2} u_{4}+u_{3} u_{4}\right) .
$$


The ODE (5.1) can be viewed as a nonlinear undamped oscillator equation with the "potential" $-Q(u)$. Since finite real-valued motion occurs only in the intervals of $u$, where $Q \geq 0$ (see figure 5.1), the structure of the solutions crucially depends on sgn $\mu$.

a)

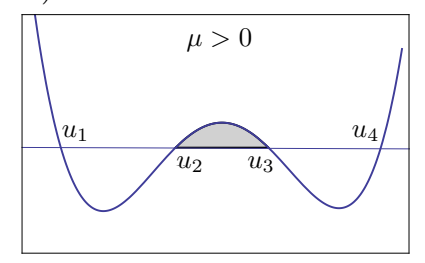

b)

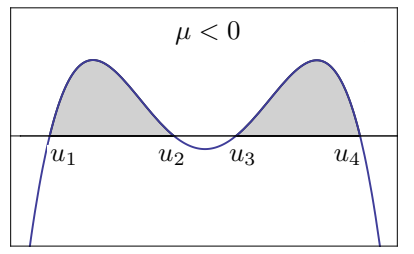

FIG. 5.1. Potential curve $Q(u)$ for traveling wave solutions of the $m K d V$ equation: (a) $\mu>0$; (b) $\mu<0$

Integration of the ODE (5.1) for both orientations of the curve $Q(u)$ shown in figure 5.1 leads generically to solutions expressed in terms of elliptic functions. The full classification of solutions arising when all four roots $u_{j}$ are real is presented in Appendix A. Some of these solutions are qualitatively analogous to the cnoidal wave solution (3.7) of the KdV equation exhibiting two distinct limits: a zero-amplitude harmonic wave and a soliton. At the same time, the fact that the potential $Q(u)$ is a quartic polynomial gives rise to a number of qualitatively new features. For example, unlike the $\mathrm{KdV}$ traveling waves, periodic solutions of the $\mathrm{mKdV}$ equation exhibit solitons of both polarities for each sign of $\mu$. Moreover, there are entirely new families of solutions not encountered in the $\mathrm{KdV}$ case. For $\mu>0$ these are kinks - smooth heteroclinic transitions connecting two equilibria $u_{1}=u_{2}$ and $u_{3}=u_{4}$ (note that $u_{1}+u_{4}=0$ due to $\left.(5.2)\right)$,

$$
u= \pm \frac{1}{2}\left(u_{4}-u_{1}\right) \tanh \left[\frac{1}{2}\left(u_{4}-u_{1}\right) \eta\right] .
$$

The traveling speed for both types of kinks is $U=u_{1}^{2}$, which agrees with the classical shock speed (4.3).

For $\mu<0$ there is a "non-KdV" family of nonlinear trigonometric solutions corresponding to the merger of two of the roots, say $u_{1}$ and $u_{2}$, while the solution oscillates between the two remaining troots $u_{3} \neq u_{4}$, see (A.13).

5.1.2. Modulation equations. The purpose of this section is to explore the structure of the mKdV modulation equations as a system of hyperbolic conservation laws. Modulation equations for the $\mathrm{mKdV}$ equation (both signs of $\mu$ ) were first derived by Driscoll and O'Neil [18] using the original Whitham averaging procedure applied to the first three $\mathrm{mKdV}$ conservation laws. The resulting system describes the slow evolution of the roots $u_{1}(x, t), u_{2}(x, t), u_{3}(x, t)$, uniquely defining the (locally) periodic solution ((A.1) for $\mu>0$ or (A.11) for $\mu<0)$ and its physical properties (period-mean, wavelength, amplitude etc.). By employing a number of non-trivial relationships between elliptic integrals it was shown in [18] that the linear combinations

$$
\lambda_{1}=\frac{1}{2}\left(u_{1}+u_{2}\right), \quad \lambda_{2}=\frac{1}{2}\left(u_{1}+u_{3}\right), \quad \lambda_{3}=\frac{1}{2}\left(u_{2}+u_{3}\right)
$$


are Riemann invariants of the mKdV modulation system. This result was inspired by the original finding of Whitham [97] for the $\mathrm{KdV}$ equation. It transpired later [82], [56], and more generally in [54] using the methods of finite-gap spectral theory [51], that the modulation system for the $\mathrm{mKdV}$ equation can be mapped onto the $\mathrm{KdV}$ modulation system (3.9). Specifically, the mKdV modulation system has the form

$$
\frac{\partial \lambda_{i}}{\partial t}+W_{i}(\boldsymbol{\lambda}) \frac{\partial \lambda_{i}}{\partial x}=0, \quad i=1,2,3
$$

where the characteristic speeds $W_{3}(\boldsymbol{\lambda}) \leq W_{2}(\boldsymbol{\lambda}) \leq W_{1}(\boldsymbol{\lambda})$ are related to the $\mathrm{KdV}$ Whitham characteristic speeds $(3.10),(3.17)$ by

$$
\begin{gathered}
\mu<0: \quad W_{i}(\boldsymbol{\lambda})=V_{4-i}(\mathbf{r}), \\
\mu>0: \quad W_{i}(\boldsymbol{\lambda})=V_{4-i}\left(\mathbf{r}^{*}\right), \quad \mathbf{r}^{*}=\left(r_{3}, r_{2}, r_{1}\right) .
\end{gathered}
$$

Here, the KdV-Whitham Riemann invariants $r_{j}$ are expressed in terms of the mKdVWhitham Riemann invariants $\lambda_{j}$ as:

$$
\begin{array}{r}
\mu<0: \quad r_{1}=3 \lambda_{3}^{2}, \quad r_{2}=3 \lambda_{2}^{2}, \quad r_{3}=3 \lambda_{1}^{2}, \\
\mu>0: \quad r_{1}=-3 \lambda_{1}^{2}, \quad r_{2}=-3 \lambda_{2}^{2}, \quad r_{3}=-3 \lambda_{3}^{2} .
\end{array}
$$

In each case $\mu>0, \mu<0$, we have for the modulus $m_{1}, m_{2}$ respectively (see (A.3), (A.12)), the same formula

$$
m_{j}=\frac{r_{2}-r_{1}}{r_{3}-r_{1}}
$$

which is identical to the KdV expression (3.8). The admissible sets of inverse formulae for $\lambda_{j}(\mathbf{r})$ (and hence for $u_{j}(\mathbf{r})$ ) are selected by the inequalities

$$
\lambda_{1} \leq \lambda_{2} \leq \lambda_{3}, \quad r_{1} \leq r_{2} \leq r_{3} .
$$

Thus, for $\mu<0$ one has either

$$
\lambda_{1}=-\sqrt{r_{3} / 3}, \quad \lambda_{2}=-\sqrt{r_{2} / 3}, \quad \lambda_{3}=-\sqrt{r_{1} / 3}
$$

or

$$
\lambda_{1}=-\sqrt{r_{3} / 3}, \lambda_{2}=-\sqrt{r_{2} / 3}, \lambda_{3}=\sqrt{r_{1} / 3} .
$$

We note that relations (5.12) correspond to modulations of the periodic solution (A.11) occurring in the interval $u_{3} \leq u \leq u_{4}$ while relations (5.13) correspond to modulations of the counterpart solution occurring in $u_{1} \leq u \leq u_{2}$.

Similarly, for $\mu>0$ one has either

$$
\lambda_{1}=-\sqrt{-r_{1} / 3}, \quad \lambda_{2}=-\sqrt{-r_{2} / 3}, \quad \lambda_{3}=-\sqrt{-r_{3} / 3}
$$

or

$$
\lambda_{1}=-\sqrt{-r_{1} / 3}, \quad \lambda_{2}=-\sqrt{-r_{2} / 3}, \quad \lambda_{3}=\sqrt{-r_{3} / 3} .
$$


The asymmetry in the expressions (5.12) and (5.13) ((5.14) and (5.15)) is due to the use of three variables $\lambda_{j}(5.5)$ replacing the four original quantities $u_{j}$ (see (5.5)), related by the condition $(5.2)$.

Non-uniqueness in the determination of the vector $\boldsymbol{\lambda}=\left(\lambda_{1}, \lambda_{2}, \lambda_{3}\right)$ for a given vector $\mathbf{r}=\left(r_{1}, r_{2}, r_{3}\right)$ is due to the invariant transformation

$$
u \rightarrow-u, \quad u_{i} \rightarrow-u_{5-i}, \quad i=1,2,3,4
$$

of the mKdV traveling wave solutions (see Appendix A) enabling two different sets of $\left\{u_{j}\right\}$ to be mapped onto the same set $\left\{r_{j}\right\}$. Indeed, one can readily see that the sets (5.12) and (5.13) (as well as (5.14) and (5.15)) are related via the transformation (5.16). The choice of the actual set $\left\{\lambda_{j}\right\}$ is determined by the initial or boundary conditions for the Whitham equations. It is also worth mentioning that the mapping $\left\{\lambda_{1}, \lambda_{2}, \lambda_{3}\right\} \mapsto\left\{r_{1}, r_{2}, r_{3}\right\}$ can be viewed as the modulation theory counterpart of the Miura transformation connecting the $\mathrm{KdV}$ and $\mathrm{mKdV}$ equations [76].

The quadratic nature of the transformations (5.9), (5.10) implies that the mKdVWhitham system (5.6) is neither genuinely nonlinear nor strictly hyperbolic. Indeed one can see from (5.7), (5.13) for $\mu<0$ and (5.8), (5.15) for $\mu>0$ that coinciding characteristic velocities are admissible: $W_{2}\left(\lambda_{1},-\lambda_{3}, \lambda_{3}\right)=W_{3}\left(\lambda_{1},-\lambda_{3}, \lambda_{3}\right)$, implying that system (5.6) is non-strictly hyperbolic. Non-genuine nonlinearity then follows $[15]$.

It is instructive to derive the non-genuine nonlinearity property of the $\mathrm{mKdV}$ Whitham system (5.6) directly, without invoking non-strict hyperbolicity.

Let $\mu<0$. Consider the derivative

$$
\frac{\partial W_{j}}{\partial \lambda_{j}}=6 \lambda_{j} \frac{\partial V_{4-j}}{\partial r_{4-j}}
$$

Since $\frac{\partial V_{j}}{\partial r_{j}} \neq 0, j=1,2,3$ due to genuine nonlinearity of the KdV-Whitham system, the derivative (5.17) can vanish only if the value $\lambda_{j}=0$ is admissible for some $j \in$ $\{1,2,3\}$. It follows from (5.12), (5.13) that one has $\lambda_{j}=0$ only when $r_{j}=0$. From the inequality $r_{1} \leq r_{2} \leq r_{3}$ and relations (5.12), (5.13) one can see that $r_{1}=0$ (i.e. $\left.\lambda_{3}=0\right)$ is admissible. Therefore the $\mathrm{mKdV}$-Whitham system is not genuinely nonlinear for $\mu<0$. The proof for $\mu>0$ is analogous and involves admissibility of $r_{3}=0$ in $(5.14),(5.15)$.

Corollary. The mKdV-Whitham system is genuinely nonlinear in any restricted $\lambda$-domain not containing an open neighbourhood of $\lambda_{3}=0$.

One can see that non-convexity of the hyperbolic flux $f(u)=u^{3}$ in the mKdV equation gives rise to complex structure of the associated modulation system, a feature not present in the classical, simple-wave DSW theory typified by the KdV DSWs described in $\S 3.2$. Indeed, the immediate consequence of non-convexity of $f(u)$ is non-genuine nonlinearity of the dispersionless limit equation (1.13), for which the characteristic velocity is $V(u)=3 u^{2}$ and so the derivative $V^{\prime}(u)=6 u$ vanishes at $u=0$. This property is naturally inherited by the $\mathrm{mKdV}$ modulation system, simply because the dispersionless equation is an exact reduction of the modulation equations obtained both in the harmonic $(m=0)$ and soliton $(m=1)$ limits (see [22]). However, the mKdV modulation system (5.6) exhibits further complexity by possessing non-strict hyperbolicity. Non-strict hyperbolicity was also observed for the modulation systems associated with the fifth order $\mathrm{KdV}$ equation (the second 
member of the integrable KdV hierarchy) [83], the complex modified mKdV equation [61] and the Camassa-Holm equation [36]. For all these equations, as well as for the mKdV equation (1.4) with $\nu=0, \mu<0$, non-strict hyperbolicity is responsible for the occurrence of a new fundamental structure, a contact $D S W$, which will be described below.

To conclude this section we note that, in spite of the same non-genuinely nonlinear inviscid/dispersionless limit shared by the mKdVB and $\mathrm{mKdV}$ equations, non-strict hyperbolicity does not enter the mKdVB TW shock theory due to the scalar nature of the problem.

5.1.3. Classical mKdV DSW solutions and admissibility conditions. The description of classical DSW solutions occurring in the Riemann problem for the $\mathrm{mKdV}$ equation requires obtaining self-similar simple-wave solutions of the modulation system, in which all but one of the Riemann invariants are constant. Such solutions exist as long as the modulation system is genuinely nonlinear (see, e.g., [64]). The mKdV DSW admissibility conditions will then include, along with Laxtype causality conditions (3.16), an extra (convexity) condition ensuring that the modulation equations are genuinely nonlinear in the relevant restricted domain of dependent variables. Due to the hyperbolic, albeit not necessarily strictly hyperbolic, nature of the problem, the necessary and sufficient condition for genuine nonlinearity is that the interval defined by the initial discontinuity (1.12) must not contain an open neighborhood of the point $u=0$. In that case, the mapping (5.9) between the $\mathrm{KdV}$ and mKdV modulations is one-to-one and the mKdV DSW modulation solution has the form of a self-similar expansion fan analogous to (3.15). To avoid ambiguity, we assume below that $u_{+} \neq 0$ and consider the special case $u_{+}=0$ separately. Also, as we shall see, the case $u_{-}=0$ does not involve DSW formation. Thus we shall be assuming that $u_{-} u_{+} \neq 0$ in our formulation of the DSW admisibility conditions.

For $\mu<0$ we have

$$
r_{1}=3 u_{+}^{2}, \quad r_{3}=3 u_{-}^{2}, \quad V_{2}\left(3 u_{+}^{2}, r_{2}, 3 u_{-}^{2}\right)=\frac{x}{t},
$$

where the connection between $r_{j}$ 's and the mKdV modulation parameters $\lambda_{j}$ is given by (5.12) or (5.13) (see the criteria below). The DSW edge speeds obtained from (5.18) are:

$$
s_{-}=3 u_{+}^{2}-3 \tilde{\Delta}, \quad s_{+}=3 u_{+}^{2}+2 \tilde{\Delta}, \quad \tilde{\Delta}=u_{-}^{2}-u_{+}^{2} .
$$

This modulation solution, inserted into the periodic TW (A.11) gives and approximate description of the mKdV DSW and is shown in figure $5.2(\mathrm{a})$ for $\left(u_{-}, u_{+}\right)=(2,1)$. Similar to the KdV DSW case, the weak limit $u(x, t, \mu)$ as $\mu \rightarrow 0$ does not coincide with the Lax shock (1.14) but is given by the period-mean value $\bar{u}(x, t)$. Also, we re-iterate that the exact phase of the TW solution (A.11) in the mKdV DSW is undetermined within the leading-order modulation theory. This does not affect the macroscopic characterization of the DSW, which is fully determined via the parameters $r_{1}, r_{2}, r_{3}$ by the modulation solution (5.18).

The solution (5.18) is subject to the admissibility conditions

$$
\left|u_{-}\right|>\left|u_{+}\right|, \quad u_{-} u_{+}>0,
$$

The meaning of inequalities (5.20) is elucidated when they are written in terms of the flux function $f(u)=u^{3}$,

$$
f^{\prime}\left(u_{-}\right)>f^{\prime}\left(u_{+}\right), \quad f^{\prime \prime}\left(u_{-}\right) f^{\prime \prime}\left(u_{+}\right)>0 .
$$


The first of the conditions (5.20) is a mKdV equivalent of the general DSW causality conditions (3.16), which guarantee that the dispersionless limit characteristics $x=$ $c_{ \pm} t+x_{0}$, where $c_{ \pm}=f^{\prime}\left(u_{ \pm}\right)=3 u_{ \pm}^{2}$, transfer initial data into the DSW region. Note that this condition does not coincide with the counterpart Lax entropy condition (4.4) for the non-convex conservation law (4.1), which says that the wave is a Lax shock if and only if $\operatorname{sgn}\left(u_{-}\right) u_{+}$lies between $-\operatorname{sgn}\left(u_{-}\right) u_{-} / 2$ and $\operatorname{sgn}\left(u_{-}\right) u_{-}$. This contrasts with the KdV case, where the DSW causality condition does coincide with the Lax entropy condition (see $\S 3.2$ ).

The second condition in (5.20) and more generally in (5.21) is the convexity condition, which guarantees that the initial step range does not include the inflection point $u=0$ of the hyperbolic flux, thus ensuring genuine nonlinearity of the modulation system for the solution involved. Therefore, the convexity condition guarantees a single-wave regularization of the initial step. One can see that for the KdV equation, the second condition (5.21) is always satisfied so that any initial jump can be regularized by a classical DSW, provided the DSW causality condition holds.

When $\operatorname{sgn}\left(u_{-}\right)=\operatorname{sgn}\left(u_{+}\right)$, this sign indicates which of the inverse mappings $(5.12)$ or (5.13) should be used to obtain the modulation $\left(\lambda_{1}, \lambda_{2}, \lambda_{3}\right)$ for the relevant periodic wave (solution (A.11) for $\mathrm{DSW}^{-}$or its reflection via (A.8) for $\mathrm{DSW}^{+}$). If $u_{-}>0$ then one needs to use relation (5.13) to describe modulations in the $\mathrm{DSW}^{+}$, while for $u_{-}<0$ one has to use $(5.12)$ to describe modulations in the $\mathrm{DSW}^{-}$. The polarity $p$ of the DSW ( see $§ 3.2)$ satisfies

$$
p=-\operatorname{sgn}\left(\mu u_{-}\right),
$$

where $p=1$ corresponds to an elevation wave and $p=-1$ corresponds to a depression wave at the DSW solitary wave edge (see §3.2). Formula (5.22) follows from the analysis of the four admissible DSW configurations (two for each sign of $\mu$ ) summarized in $\$ 5.2 .1,5.2 .2$ below.

In more general terms,

$$
p=-\operatorname{sgn}\left(\mu f^{\prime \prime}\left(u_{-}\right)\right) .
$$

The behaviors of characteristics for the modulation system in the DSW region are analogous to those for the $\mathrm{KdV}$ case as in figure 3.3. However, KdV exhibits only the $\mathrm{DSW}^{+}$for $\mu<0$ (see figure 3.5). In contrast, (5.23) demonstrates that mKdV's non-convex flux allows for both $\mathrm{DSW}^{+}$and $\mathrm{DSW}^{-}$regularizations without changing the dispersion sign. It is the product of the dispersion convexity and the hyperbolic flux convexity in (5.23) that determines a DSW's polarity. On the other hand, the DSW orientation (see $§ 3.2$ ) is determined by the dispersion sign alone. For the mKdV equation with a fixed dispersion sign, $\mathrm{DSW}^{+}$and $\mathrm{DSW}^{-}$have the same orientation, i.e. the same relative positions (trailing, leading) of the solitary wave and harmonic edges.

The mKdV DSW modulation solution for the case $\mu>0$ is obtained from (5.18) by applying the transformation (3.17) and then using one of the inverse mappings (5.14), (5.15) to get the modulation $\left(\lambda_{1}, \lambda_{2}, \lambda_{3}\right)$ of the periodic solution (A.1). One uses the mapping (5.14) for a $\mathrm{DSW}^{+}$and (5.15) for a $\mathrm{DSW}^{-}$. The DSW admissibility conditions have the same form (5.20) but the DSW polarity is opposite to that occurring in the case $\mu<0$, see (5.22). The speeds of the trailing and leading edges for the DSWs of both polarities are (cf. (5.19)): $s_{-}=3 u_{+}^{2}+\tilde{\Delta}, s_{+}=3 u_{+}^{2}+6 \tilde{\Delta}$.

In all cases, the leading $(\mu<0)$ or trailing $(\mu>0)$ soliton amplitude in the classical DSW is $a=2\left(\left|u_{-}\right|-\left|u_{+}\right|\right)$. 
a)

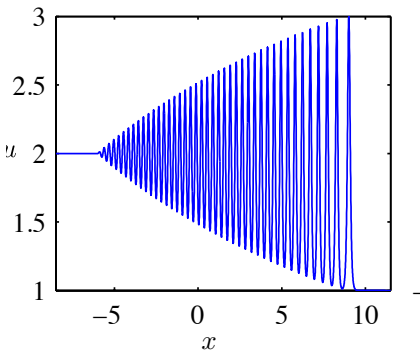

b)

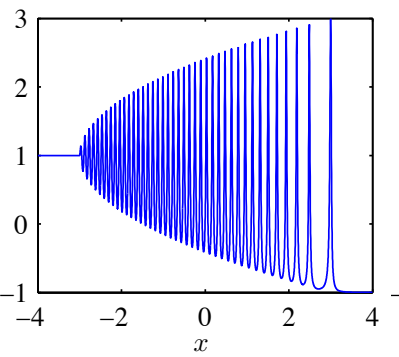

c)

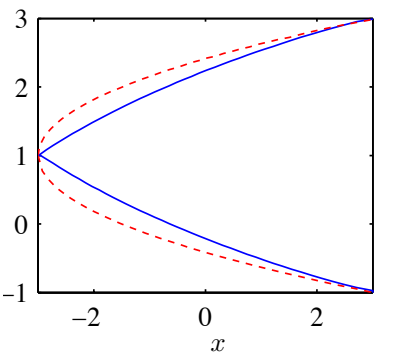

FIG. 5.2. Dispersive shock waves for the $m K d V$ equation, constructed at $t=1$ from $m K d V$ modulation theory. (a) Classical DSW, $\mu=-2 \cdot 10^{-2}$. (b) Non-Classical contact DSW, $\mu=-5 \cdot 10^{-4}$. (c) Envelopes of the DSW (solid, martini glass shape, scaled for comparison) and the CDSW (dashed, bordeaux wine glass shape).

If one or both of the admissibility conditions (5.20) fail for a given pair $\left(u_{-}, u_{+}\right)$, then the regularization of a step (1.12) via a single classical DSW is not possible. If only the DSW causality condition is violated but the convexity condition holds true, then the dispersive resolution occurs via a rarefaction wave. The $\mathrm{mKdV}$ rarefaction wave solutions for $\left|u_{+}\right|>\left|u_{-}\right|$for $t \gg 1$ have the leading order asymptotic form (4.11), same as for the mKdVB equation (1.4). In contrast to the counterpart rarefaction wave solution of the $\mathrm{mKdVB}$ equation, the corners of the $\mathrm{mKdV}$ rarefaction wave are smoothed by linear dispersive oscillations whose wavelength $\sim \sqrt{|\mu|}$ and amplitude decays like $t^{-1 / 2}[66]$, similar to the asymptotic behavior in the $\mathrm{KdV}$ rarefaction wave described in Sec. 3.2. Note that only the $\mu>0$ case is discussed in [66].

In the remaining two $(\mu<0$ and $\mu>0)$ classical DSW-inadmissible cases involving the breaking of the convexity condition, "non-classical" dispersive regularizations occur; these will be described in the following subsections. The borderline case when $u_{+}=0$ can be formally viewed as being of either classical or non-classical regularization type, and will be discussed briefly.

5.1.4. Non-classical DSWs. We first consider the case $\mu>0$. Here, we already have an exact heteroclinic solution to the mKdV equation - the kink solution (5.4) - that violates the convexity condition. Indeed, the kink with the minus sign in (5.4) connects two states $u_{-}>0$ at $x \rightarrow-\infty$ and $u_{+}=-u_{-}$at $x \rightarrow \infty$ so that $u_{-} u_{+}<0$. The antikink with the plus sign in (5.4) is the reflection of the kink under the transformation (A.8) and has the same non-convexity property. One can see that kinks violate both the DSW causality (5.20) and Lax entropy (4.2) conditions as well. Unlike the classical, expanding, DSW, the kink is a moving front approximating a shock wave, so must be evaluated against the Lax entropy condition. The Lax entropy condition agrees with the counterpart DSW causality condition (3.16) if one takes $s_{-}=s_{+}=s_{K}$. However, the kink speed is $s_{\mathrm{K}}=u_{-}^{2}<f^{\prime}\left(u_{ \pm}\right)=3 u_{-}^{2}=3 u_{+}^{2}$ so that kinks are undercompressive as described in $\S(1.15)$. From the discussion of traveling waves corresponding to undercompressive shocks in $\S 5.1 .1$, we observe that the kink solution (A.9) is the strong $\delta \rightarrow 0$ limit of the diffusive-dispersive undercompressive shock solution (4.9), (4.10). Thus we identify kinks as undercompressive DSWs. Note that the undercompressive nature of $\mathrm{mKdV}$ kinks was discussed in [59]. In Sec. 5.2, we will incorporate them into the general context of the mKdV Riemann problem classification. 
An exploration of solutions to the Riemann problem (1.12) for the mKdV equation with $\mu>0$ was undertaken in [11]. The amplitude of the DSW's lead soliton was determined using an inverse scattering approach and the necessary and sufficient condition $u_{-} u_{+}<0$-breaking of the convexity condition (5.20)-for the formation of a kink (termed a double-layer solution) was identified. Numerical simulations were used to identify kinks, classical DSWs, rarefaction waves, and double wave combination solutions. In the next section, we will provide the full classification of the mKdV Riemann problem for both $\mu>0$ and $\mu<0$.

The Riemann problem (1.12) for the mKdV equation with $\mu>0$ and the particular initial data $u_{+}=-u_{-}$was studied in [66], where a detailed solution was obtained using matched asymptotic expansions. The long-time asymptotics of this solution is dominated by a kink, which agrees with the modulation theory approach where kinks are used as one of the 'building blocks' in the construction of long-time asymptotic solutions in more general Riemann problems, see [26], [54].

Now we turn to the case $\mu<0$. Instead of kinks, a peculiar type of DSW occurs, which we term a contact DSW (CDSW). A CDSW, similar to a kink, connects two conjugate states $u_{-}>0$ at $x \rightarrow-\infty$ and $u_{+}=-u_{-}$at $x \rightarrow \infty$ (this is $\mathrm{CDSW}^{+}$) but, unlike the kink, has an oscillatory structure resembling that of a classical DSW. Contact DSWs represent the dispersive counterparts of single-sided contact discontinuities (or sound shocks) known in the theory of hyperbolic conservation laws (see, e.g., $[15])$.

The $\mathrm{CDSW}^{+}$solution for the mKdV equation is described by the special modulation $\lambda_{1}, \lambda_{2}, \lambda_{3}$ in which $\lambda_{2}=\lambda_{3}$ so that the elliptic parameter $m=0$ (see (5.11)) throughout the wave train, but the wave amplitude $a=u_{4}-u_{3}=-4 \lambda_{3} \geq 0$. This sharply contrasts with KdV modulation theory where $m=0$ implies zero wave amplitude. The $\mathrm{CDSW}^{+}$modulation solution has the form

$$
\begin{aligned}
-\lambda_{1} & =u_{-}, \quad \lambda_{2}=\lambda_{3}, \\
W_{2}\left(-u_{-}, \lambda_{3}, \lambda_{3}\right) & =W_{3}\left(-u_{-}, \lambda_{3}, \lambda_{3}\right)=\frac{x}{t},
\end{aligned}
$$

or explicitly, using (5.7), (3.12) and (5.9),

$$
\lambda_{3}=-\frac{1}{\sqrt{6}} \sqrt{\frac{x}{t}+3 u_{-}^{2}} .
$$

Since $\lambda_{2}=\lambda_{3}$ implies $u_{1}=u_{2}$ and the oscillations in the $\mathrm{CDSW}^{+}$are confined to the interval $u_{3} \leq u \leq u_{4}$, we conclude that the 'carrier' wave in a $\mathrm{CDSW}^{+}$is the nonlinear trigonometric solution (A.13), and that is why this type of DSW has been termed a sinusoidal or trigonometric DSW elsewhere [75], [54]. At the leading edge of a $\operatorname{CDSW}^{+}, \lambda_{1}=\lambda_{2}=\lambda_{3}$ (i.e. $u_{1}=u_{2}=u_{3}$ ), which manifests a 'bright' algebraic soliton (A.14) with amplitude $a=-4 \lambda_{1}=4 u_{-}$and speed $U=3 \lambda_{1}^{2}$. The algebraic soliton propagates on the background $\bar{u}=\lambda_{1}$ which, according to the matching regularization conditions, must be matched to the constant state $u=u_{+}$upstream. Then (5.24) yields that a $\mathrm{CDSW}^{+}$can only connect the conjugate states $u=u_{-}>0$ and $u=u_{+}=-u_{-}<0$. At the $\mathrm{CDSW}^{+}$trailing edge, the wave amplitude $a=0$, i.e., $\lambda_{2}=\lambda_{3}=0$. Thus, the speeds of the $\mathrm{CDSW}^{+}$trailing and leading edges are: $s_{-}=W_{2}\left(-u_{-}, 0,0\right)=-3 u_{-}^{2}, s_{+}=W_{2}\left(-u_{-},-u_{-},-u_{-}\right)=3 u_{-}^{2}=3 u_{+}^{2}$. This modulation solution, inserted into the periodic TW (A.13), is shown in figure 5.2(b). Similar to the classical KdV and mKdV DSWs, the exact phase of the CDSW is not determined by the modulation solution (5.24). 
The fundamental difference between the modulation solution (5.18) for a classical DSW and the solution (5.24) for a CDSW is that the solution of (5.24) represents a double characteristic fan, the corresponding distinct Riemann invariants being $\lambda_{3}$ and $-\lambda_{2}$. This solution is possible owing to the nonstrict hyperbolicity of the mKdV modulation system. Note that the trigonometric structure of the underlying mKdV traveling wave solution (A.13) is not the defining feature of CDSWs. See [61] where a qualitatively similar type of modulation occurs for a wave with $m \neq 0$. The more fundamental contact property is that the CDSW leading edge is a triple characteristic, tangential to the external dispersionless characteristic with characteristic velocity $3 u^{2}$. As a result, a $\mathrm{CDSW}^{+}$could be matched with a rarefaction wave at the leading edge. The composite CDSW-RW solutions will be considered in $§ 5.2 .2$. We also mention the qualitative difference between the appearances of the envelopes for classical and contact DSWs: the classical DSW typically has a "martini-glass" shape due to the asymptotic behavior of the amplitude $a \sim\left(x-x_{-}\right)$near the harmonic edge at $x=x_{-}=s_{-} t$, whereas the CDSW envelope defined by $a=-4 \lambda_{3} \sim \sqrt{x-x_{-}}$has a "Bordeaux-glass" shape as shown in figure 5.2(c). We note that these envelope distinctions may not be obvious in direct numerical simulations due to contributions not included in the Whitham multiple-scale solution, which could become important in the vicinity of the harmonic edge defining the appearance of the envelope. See [34] for the corresponding $\mathrm{KdV}$ analysis of higher order corrections to the harmonic edge.

The description of a $\mathrm{CDSW}^{-}$, where the oscillations occur between the other pair of roots, $u_{1} \leq u \leq u_{2}$, is analogous and involves the reflection of the traveling wave formulae (A.11) - (A.14) via the transformation (A.8) and the relationships (5.13) for the modulation variables.

5.2. Riemann problem classification. The Riemann problem classification for the $\mathrm{mKdV}$ equation on the $u_{-}-u_{+}$plane of initial data is constructed by considering the admissibility conditions (5.20) and all the cases when one or both of them fail. As a result, the $u_{-}-u_{+}$plane is divided into eight equi-spaced sectors, each corresponding to a distinct fundamental wave regularization pattern (see figure 5.3). The separation lines are: $u_{-}=0, u_{+}=0, u_{+}=u_{-}$and $u_{+}=-u_{-}$. Due to the symmetry inherent in the causality and convexity conditions (5.20), the boundaries between different solution types are symmetric under a $\pi / 4$ rotation.

5.2.1. Classification for $\mu>0$. The classification is shown in figure 5.3(a). We have already considered solutions corresponding to the classical DSW admissible regions defined by the conditions (5.20). The adjacent regions with $\left|u_{-}\right|<\left|u_{+}\right|$, where the DSW causality condition fails but the convexity condition holds, correspond to regularization via rarefaction waves described by (4.11). The line $u_{+}=-u_{-}$corresponds to a step regularization via a single kink $\left(u_{-}>0\right)$ or antikink $\left(u_{-}<0\right)$. These have also been described in $\S 5.1 .4$. We now need to describe the remaining two cases corresponding to the sectors $-u_{-}<u_{+}<0$ and $u_{+}<-u_{-}<0$. Conjugate solutions corresponding to the opposite sectors are obtained via the reflection transformation (A.8).

Let $-u_{-}<u_{+}<0$. The Riemann problem solution is a double-wave $\mathrm{K} \mid \mathrm{DSW}^{+}$ consisting of a kink and a $\mathrm{DSW}^{+}$connected by the constant state $u=-u_{-}$. The kink speed $s_{\mathrm{K}}=u_{-}^{2}$, and the speeds of the $\mathrm{DSW}^{+}$trailing and leading edges are $s_{-}=u_{-}^{2}+2 u_{+}^{2}$ and $s_{+}=6 u_{-}^{2}-3 u_{+}^{2}$ respectively. One can see that $s_{-}>s_{\mathrm{K}}$ so the interval between the kink and $\mathrm{DSW}^{+}$increases with time.

If $u_{+}<-u_{-}<0$, then the initial step regularization occurs via a kink and a 


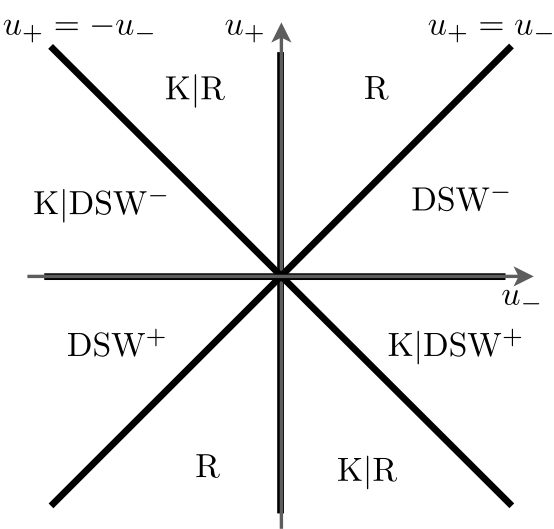

a) $\mu>0, \nu=0$

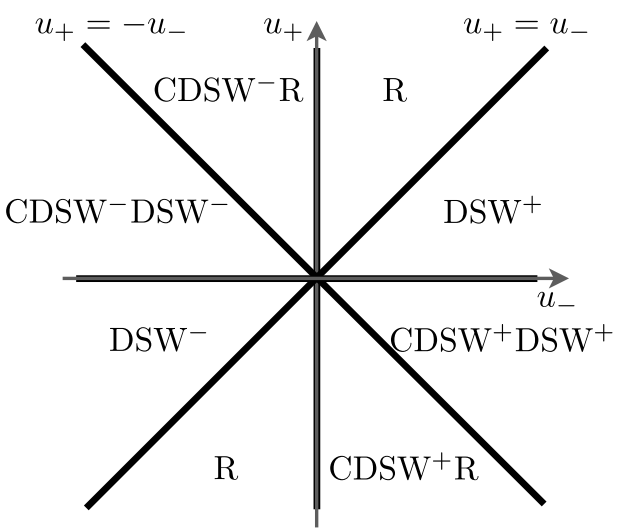

b) $\mu<0, \nu=0$

FIG. 5.3. Solutions of the Riemann problem for the $m K d V$ equation. Legend: $(R)$ - rarefaction wave; $\left(D S W^{+}\right)$- 'bright' $D S W ;\left(D S W^{-}\right)$- 'dark' $D S W ; K-k i n k ;\left(C D S W^{ \pm}\right)$- 'contact' $D S W^{ \pm}$; (l) - intermediate constant state.

rarefaction wave (formula (4.11) with the minus sign, and $u_{-}$replaced with $-_{-} u_{-}$) connected by a widening interval in which $u=-u_{-}$.

It remains to explain the singular transition between the patterns $\mathrm{DSW}^{-}$for $0<u_{+}<u_{-}$and $\mathrm{K} \mid \mathrm{DSW}^{+}$for $-u_{-}<u_{+}<0$, which occurs when crossing the horizontal axis $u_{+}=0$. The trailing oscillation in the $\mathrm{DSW}^{-}$represents a depression (dark) soliton (A.6) having the amplitude $2\left(\left|u_{-}\right|-\left|u_{+}\right|\right)$and propagating with the speed $s_{-}=2 u_{+}^{2}+u_{-}^{2}$. When $u_{+}=0$ the soliton speed becomes equal to the kink speed $s_{\mathrm{K}}=u_{-}^{2}$, and the left slope of the dark soliton becomes equivalent to a kink. The further decrease of $u_{+}$results in a 'peeling' off of the trailing kink from the remaining wave train, which becomes a $\mathrm{DSW}^{+}$. See [54] for a series of numerical simulations illustrating the analogous transition for the Gardner equation.

5.2.2. Classification for $\mu<0$. The classification of the Riemann problem solution to the $\mathrm{mKdV}$ equation with $\mu<0$ is shown in figure $5.3(\mathrm{~b})$. The classical DSW regularizations corresponding to two opposite sectors of the $u_{-}-u_{+}$plane where the admissibility conditions $(5.20)$ hold were described in $\S 5.1 .3$. Similar to the $\mu>0$ case, the adjacent regions with $\left|u_{-}\right|<\left|u_{+}\right|$, where the DSW causality condition fails but the convexity condition holds, correspond to regularization via rarefaction waves described by (4.11). The line $u_{+}=-u_{-}$corresponds to a step regularization via a single $\mathrm{CDSW}^{+}\left(u_{-}>0\right)$ or $\mathrm{CDSW}^{-}\left(u_{-}<0\right)$. These have also been described in \$5.1.4. We now need to describe the remaining two cases corresponding to the sectors $-u_{-}<u_{+}<0$ and $u_{+}<-u_{-}<0$. The conjugate solutions corresponding to the opposite sectors are obtained via the reflection transformation (5.16).

Let $-u_{-}<u_{+}<0$. The Riemann problem solution is a compound wave $\mathrm{CDSW}^{+} \mathrm{DSW}^{+}$consisting of a partial $\mathrm{CDSW}^{+}$and $\mathrm{DSW}^{+}$attached to each other. The line separating the $\mathrm{CDSW}^{+}$and the $\mathrm{DSW}^{+}$in the $x-t$ plane is the characteristic $s_{*}=6 u_{+}^{2}-3 u_{-}^{2}$. The trailing edge of the compound wave $\mathrm{CDSW}^{+} \mathrm{DSW}^{+}$is $s_{-}=-3 u_{-}^{2}$. The leading edge is $s_{+}=2 u_{-}^{2}+u_{+}^{2}$.

If $u_{+}<-u_{-}<0$, then initial step regularization occurs via a combination of a fully developed $\mathrm{CDSW}^{+}$connected at the leading edge to a rarefaction wave. 
The classification of the regularization patterns in terms of the admissibility/nonadmissibility conditions for both signs of $\mu$ is presented in Table 5.1.

\begin{tabular}{|c|c|c|}
\hline Causality $\left(\left|u_{-}\right|>\left|u_{+}\right|\right)$ & Convexity $\left(u_{-} u_{+}>0\right)$ & Regularization $\mu>0(\mu<0)$ \\
\hline Yes & Yes & $\mathrm{DSW}^{-}\left(\mathrm{DSW}^{+}\right)$ \\
\hline No & Yes & $\mathrm{R}(\mathrm{R})$ \\
\hline Yes & No & $\mathrm{K} \mid \mathrm{DSW}^{+}\left(\mathrm{CDSW}^{+} \mid \mathrm{DSW}^{+}\right)$ \\
\hline No & No & $\mathrm{K} \mid \mathrm{R}\left(\mathrm{CDSW}^{+} \mid \mathrm{R}\right)$ \\
\hline \multicolumn{2}{|c}{ TABLE 5.1}
\end{tabular}

Classification of regularization patterns in terms of satisfaction/violation of the admissibility conditions (5.20) for $u_{-} u_{+} \neq 0$. The DSW polarity is shown for $u_{-}>0$.

6. Comparison. As can be seen by the very different analytical approaches outlined in $\S 4$ and $\S 5$, the Riemann problem solutions in the presence of dispersion with or without diffusion evolve in fundamentally different ways. However, the results do exhibit direct parallels that provide a useful bridge connecting diffusive-dispersive $\mathrm{mKdVB}$ dynamics to purely dispersive $\mathrm{mKdV}$ dynamics. It is our aim in this section to identify the key differences and commonalities between these two models.

6.1. Shock structure. The first and perhaps most obvious distinction between the two model types can be seen in the spatial structure of the solutions themselves. Figures 6.1 and 6.2 display numerical solutions to smoothed versions of the Riemann problem when $\mu=1$ and $\mu=-1$, respectively. See the Appendix for a description of the numerical method used. The first column of panels in each figure corresponds to purely dispersive dynamics with $\nu=0$, whereas the second column corresponds to diffusive-dispersive dynamics in which $\nu>0$. The text labels adjacent to each panel correspond to the classification in the $\left(u_{-}, u_{+}\right)$regions of figures 4.2, 5.3. Due to the invariance of (1.4) under the transformation $u \rightarrow-u$, we restrict attention to the choice $u_{-}<u_{+}$, understanding that the analogous regimes when $u_{-}>u_{+}$exhibit an amplitude reflection so ${ }^{+}$waves become ${ }^{-}$waves and vice-versa.

Many of the solutions exhibit oscillations due to the presence of dispersion. The presence of any diffusion damps the oscillation amplitudes. In contrast, the purely dispersive case leads to the continual generation of large amplitude oscillations showcasing the distinction between diffusive traveling waves approximating Lax shocks and expanding, dynamic DSWs. Even the rarefactions are subject to larger oscillation in the absence of diffusion. Notice, however, that both undercompressive shocks and kinks are monotone, exhibiting no oscillation.

Due to the existence of two speeds, DSWs exhibit an orientation distinguishing the large amplitude, soliton edge from the small amplitude, harmonic wave edge. Another DSW characteristic is polarity, determined by the type of disturbance (elevation or depression) occurring at the soliton edge. Both orientation and polarity are inherited from the corresponding diffusive-dispersive wave when $\delta=\nu / \sqrt{|\mu|}$ is sufficiently small (recall $\S 4.2$ ), as shown in figures 6.1 and 6.2. The DSW orientation is determined by the sign of dispersion. As in the KdV equation, the only way to change the polarity of a DSW for a convex conservation law is to change the sign of dispersion, recall (5.23). A non-convex flux introduces the possibility of polarity change without changing the dispersion sign, as in figure 6.1(a). This can have physically important implications e.g. in internal wave dynamics (the distinction between dam-break and lock-exchange undular bores [26]) or in nonlinear optics/superfluids (the occurrence of bright solitons in a defocusing medium [14]). 

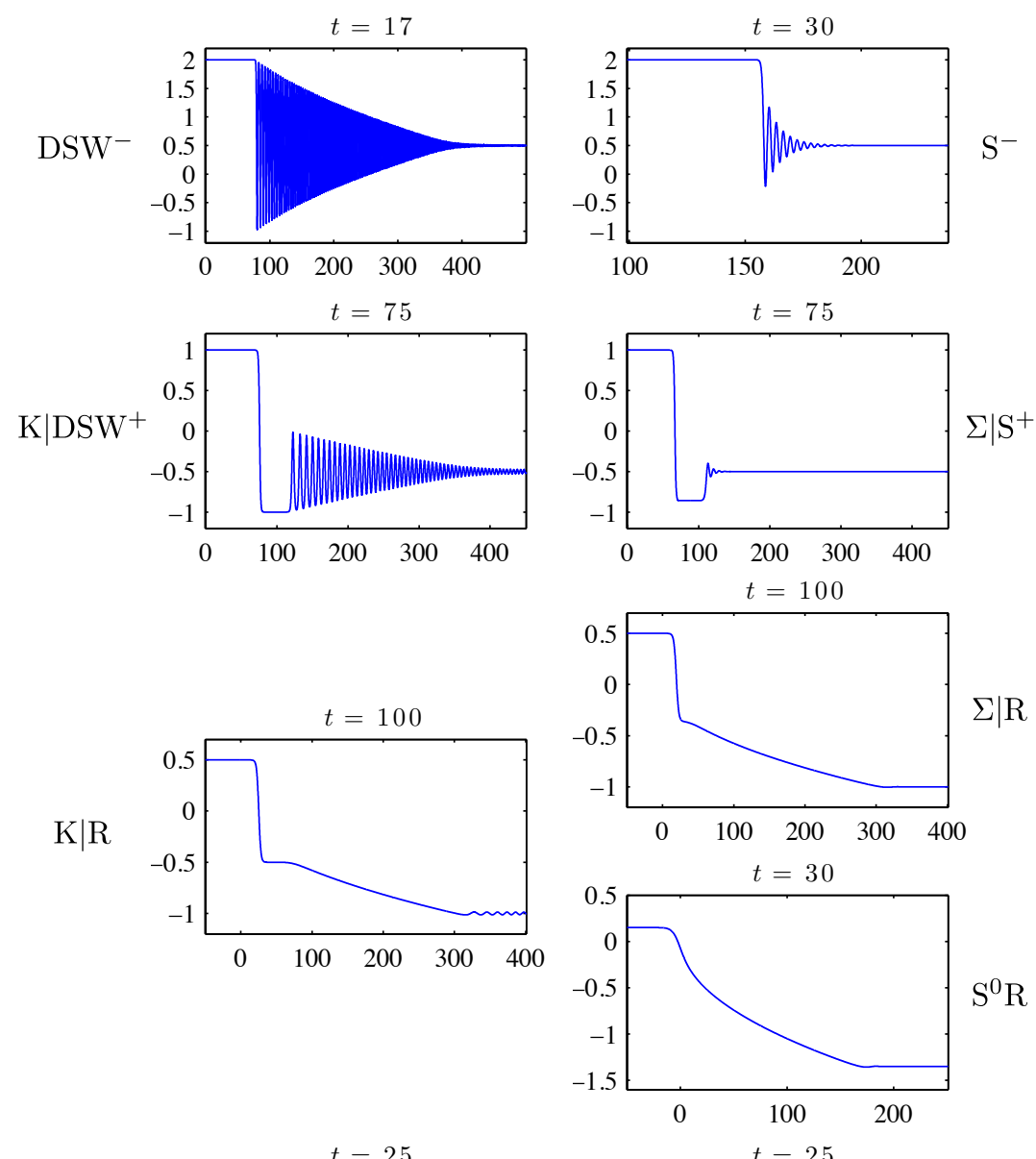

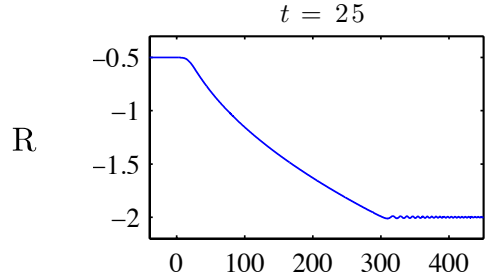

a) $\mu=1, \nu=0$

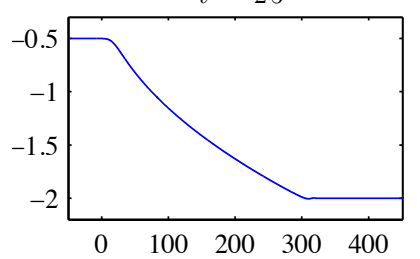

b) $\mu=1, \nu=0.3$

FIG. 6.1. Numerical simulations of the Riemann problem for (a) mKdV and (b) mKdVB, with positive dispersion. The panel labels identify the types of waves, as in figures 4.2 and 5.3.

6.2. Characteristic diagrams. A useful means for understanding different wave solutions is through their characteristic diagrams. For scalar conservation laws, there is only one characteristic family. For the diffusive-dispersive case, this single characteristic family is sufficient to illustrate the TW solutions. However, in the purely dispersive case, shock solutions are described by a modulated, periodic TW via the Whitham equations with three distinct characteristic families. The characteristic description of single rarefactions, Lax shocks, and classical DSWs for the $\mathrm{mKdV}(\mathrm{B})$ 


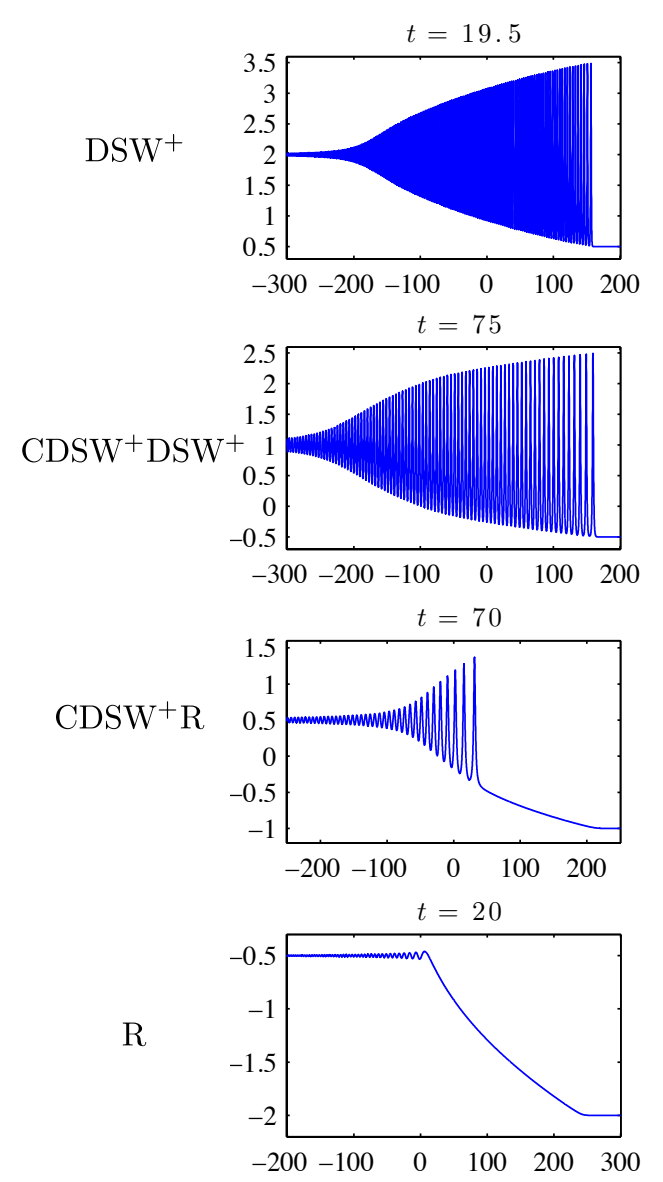

a) $\mu=-1, \nu=0$

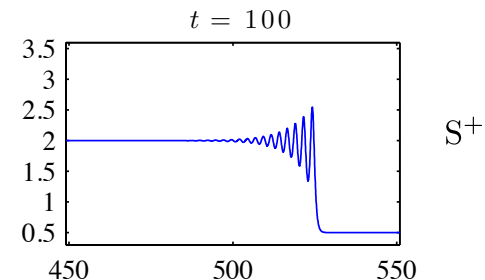

$\mathrm{S}^{+}$
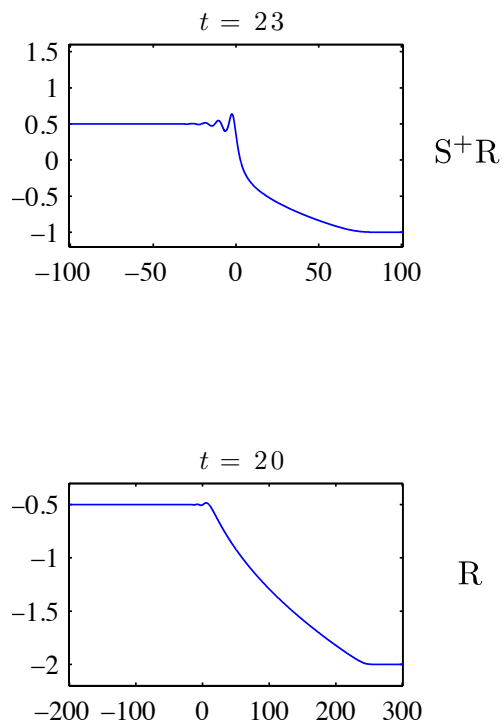

b) $\mu=-1, \nu=0.3$

FIG. 6.2. Numerical simulations of the smoothed Riemann problem for (a) mKdV and (b) $m K d V B$, with negative dispersion. The panel labels identify the types of waves, as in figures 4.2 and 5.3.

equation is similar to that for the $\mathrm{KdV}(\mathrm{B})$ equation shown in figure 3.3.

Figures $6.3,6.4$, and 6.5 display characteristic diagrams and modulation solutions for double wave structures in the $\mathrm{mKdV}(\mathrm{B})$ Riemann problem classifications. The mKdV-Whitham characteristic families are denoted by $\Gamma_{i}, i=1,2,3$ where $\Gamma_{i}$ is the set of characteristic curves $x=x(t)$ satisfying $\frac{\mathrm{d} x}{\mathrm{~d} t}=W_{i}$, with $W_{i}$ the characteristic velocity in (5.6). Although we display generic diagrams, each characteristic is a legitimate one computed for a specific Riemann problem, i.e., these are not sketches. We now describe the implications of each of these diagrams.

Figure 6.3 compares the shock-rarefaction, $\mathrm{S}^{+} \mathrm{R}$ in (a), and the contact DSWrarefaction, denoted $\mathrm{CDSW}^{+} \mathrm{R}$ in (b-d), operable in the $\mu<0$ regime. Both the shock and the leading edge of the CDSW coincide with the trailing characteristic in the rarefaction, with speed $s_{+}$. Moreover, the CDSW leading edge is a triple characteristic, i.e., $s_{+}=W_{1}=W_{2}=W_{3}$. Here, all three modulation characteristics coincide, the reason for the terminology contact DSW. An additional feature of 
a) $\mathrm{S}^{+} \mathrm{R}$

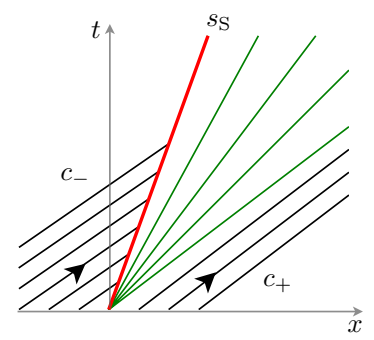

b) $\quad \mathrm{CDSW}^{+} \mathrm{R}$

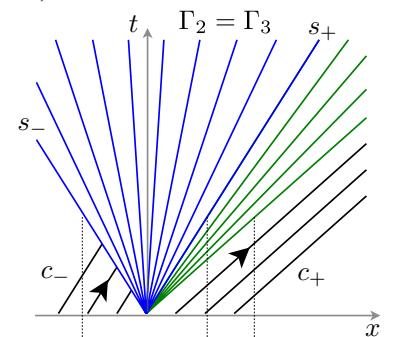

d)

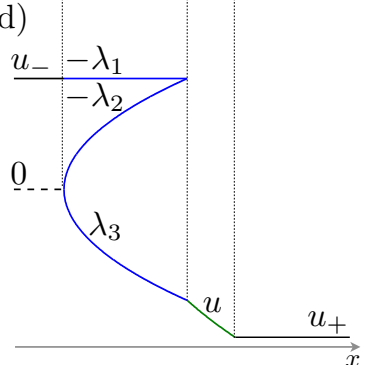

c)

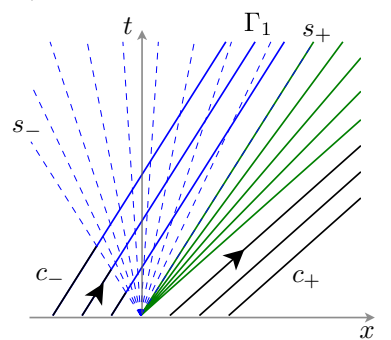

FIG. 6.3. Characteristic diagrams for the composite shock-rarefaction (a) and contact DSWrarefaction $(b, c)$ solutions. The modulation solution $(d)$ and associated three characteristic families $\Gamma_{1}, \Gamma_{2}$, and $\Gamma_{3}(b, c)$ are shown. The dashed curves correspond to $\Gamma_{2}=\Gamma_{3}$.

the CDSW distinguishing it from the classical DSW (recall figure 3.3) is the double characteristic family $\Gamma_{2}=\Gamma_{3}$, maintained across the entire contact dispersive shock structure. The remaining characteristic family passes straight through the CDSW, unchanged from the dispersionless characteristics with speed $c_{-}$, so that $c_{-}=s_{+}$also.

Figure 6.4 depicts a $\mathrm{CDSW}^{+} \mathrm{DSW}^{+}$and allows for a side-by-side comparison of the non-classical CDSW and classical DSW characteristics. In this double wave structure, the CDSW modulation solution exhibits double characteristics $W_{2}=W_{3}$ up to its interface with the DSW where $s_{*}=W_{2}=W_{3}$. The $\Gamma_{1}$ characteristic family carries information through the CDSW with speed $c_{-}$and into the DSW. The CDSW modulation solution (5.24) is unfolded by considering the alternative Riemann invariants $\lambda_{3} \leq-\lambda_{2} \leq-\lambda_{1}$ as in figures $6.3(\mathrm{~d})$ and $6.4(\mathrm{~d})$. This choice is natural for the $\mathrm{CDSW}^{+}$because its corresponding periodic TW solution (A.13) satisfies $u_{3} \leq u \leq u_{4}$ (recall figure 5.1). Our original choice of Riemann invariants (5.5) is more suited for TWs with $u_{1} \leq u \leq u_{2}$.

When $\mu>0$, composite waves involve undercompressive shocks, denoted $\Sigma$, for $\mathrm{mKdVB}$, or kinks, denoted $\mathrm{K}$, for $\mathrm{mKdV}$. Their characteristics are shown in figure 6.5. In contrast to the $\mu<0$ double wave solutions, each wave in the composite solution for $\mu>0$ is separated by an intermediate constant state, the separation indicated by a vertical line $\mid$. The shock-rarefaction solution $\mathrm{S}^{0} \mathrm{R}$ for $\mathrm{mKdVB}$, not displayed, is an exception in not having an intermediate constant; the speed of $\mathrm{S}^{0}$ coincides with the characteristic speed $c_{-}$and forms the trailing edge of the rarefaction wave. A defining property of undercompressive shocks is the passage of a characteristic family through them. Figures 6.5(a,c) show how characteristics enter the undercompressive shock from the left and exit on the right with an upward deflection. In contrast, the corresponding kink solutions in figures $6.5(\mathrm{~b}, \mathrm{~d})$ exhibit characteristics that pass 
a)

b) $\mathrm{CDSW}^{+} \mathrm{DSW}^{+}$

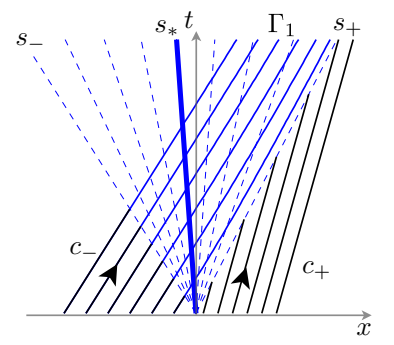

$\Gamma_{2}=\Gamma_{3} s_{*} \quad \Gamma_{2} \quad s_{+}$

c)

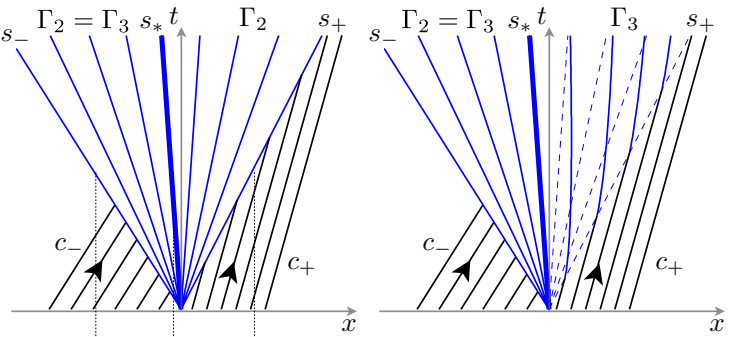

d)

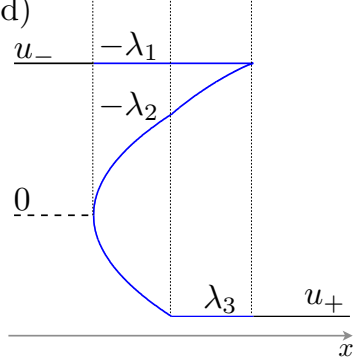

FIG. 6.4. Characteristic diagrams for the three characteristic families $\Gamma_{1}, \Gamma_{2}$, and $\Gamma_{3}$ of the composite CDSW-DSW solution (a-c). The modulation solution is in (d). The dashed curves correspond to either $\Gamma_{2}=\Gamma_{3}$ (between $s_{-}$and $s_{*}$ ) or $\Gamma_{2}$ (between $s_{*}$ and $s_{+}$).

through with no deflection, just like one of the characteristic families for the CDSW solutions in figures 6.3(c) and 6.4(a). Through this similarity, the CDSW can also be thought of as undercompressive, however without a diffusive counterpart. Unlike the DSW and CDSW, the kink is a genuine, non-modulated TW solution of mKdV. Its effect in the modulation description of the $\mathrm{K} \mid \mathrm{DSW}^{+}$of figure $6.5(\mathrm{e})$ is a discontinuous jump from $u_{-}$to $-u_{-}$, the intermediate constant connecting to the classical DSW. The $\mathrm{K}_{\mid \mathrm{DSW}}^{ \pm}$is the only mKdV Riemann problem solution that incorporates both modulated (DSW) and non-modulated (kink) TWs.

6.3. Zero Diffusion Limit. The limit $\nu \rightarrow 0^{+}$for solutions of the mKdVB equation is singular in that for every nonzero $\nu$, the long time shock behavior of the Riemann problem is resolved into a heteroclinic orbit or TW (4.9), (4.10). Diffusion introduces a relaxation mechanism to a steady configuration: TWs are attractors. But when $\nu=0$, the only heteroclinic orbit available is the kink, valid only for very specific left and right states when $\mu>0$. So, the purely dispersive regularization for generic left and right states results in dynamic, oscillatory coherent structures. DSWs are unsteady, multiscale attractors that combine a periodic TW with an additional layer of self-similarity, the modulation solution. Nevertheless, we can see from the numerical results in figures 6.1 and 6.2 a correspondence between $\nu>0$ and $\nu=0$ in most cases. It is therefore natural to introduce a mapping of the $\nu>0 \mathrm{mKdVB}$ solutions to $\nu=0 \mathrm{mKdV}$ solutions. As we will see, this mapping is multivalued and not one-to-one.

We define the zero diffusion mapping for the Riemann problem by the classification figures $4.2(\nu>0)$ and $5.3(\nu=0)$ reproduced in figure 6.6. Common domains in the $u_{-}-u_{+}$plane demonstrate how diffusive-dispersive wave solutions map to the purely dispersive case. The mapping is summarized in table 6.1. The first and third 

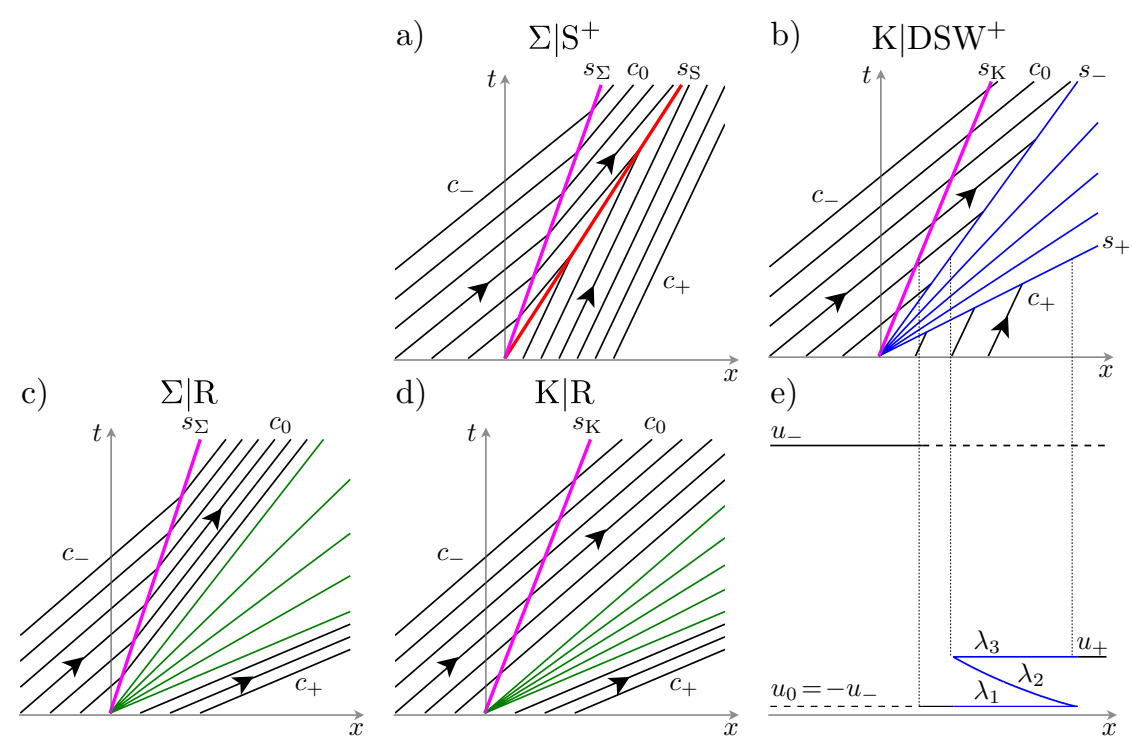

FIG. 6.5. Characteristic diagrams for Riemann problem solutions of $m K d V B(a, c)$ and $m K d V$ $(b, d)$ with $\mu>0$. The $D S W$ modulation solution for Riemann invariants is shown in (e).

\begin{tabular}{|c|c|}
\hline negative dispersion $\mu<0$ & positive dispersion $\mu>0$ \\
\hline $\mathrm{R} \rightarrow \mathrm{R}$ & $\mathrm{R} \rightarrow \mathrm{R}$ \\
\hline $\mathrm{S}^{ \pm} \rightarrow\left\{\begin{array}{c}\mathrm{DSW} \\
\text { or } \\
\mathrm{CDSW}^{ \pm} \mathrm{DSW}^{ \pm}\end{array}\right.$ & $\mathrm{S}^{ \pm} \rightarrow\left\{\begin{array}{c}\mathrm{DSW} \\
\text { or } \\
\mathrm{K} \mid \mathrm{DSW}^{ \pm}\end{array}\right.$ \\
\hline $\mathrm{S}^{ \pm} \mathrm{R} \rightarrow\left\{\begin{array}{c}- \\
\mathrm{CDSW}^{ \pm} \mathrm{R} \\
\text { or } \\
\mathrm{CDSW}^{ \pm} \mathrm{DSW}^{ \pm}\end{array} \begin{array}{c}\Sigma \mid \mathrm{R} \\
\text { or } \\
\mathrm{S}^{0} \mathrm{R}\end{array}\right\} \rightarrow\left\{\begin{array}{c}\mathrm{K} \mid \mathrm{R} \\
\text { or } \\
\mathrm{K} \mid \mathrm{DSW}^{ \pm}\end{array}\right.$ \\
\hline \multicolumn{2}{|c|}{ TABLE 6.1}
\end{tabular}

Zero diffusion limit mapping Riemann problem solutions of $m K d V B$ to those of $m K d V$.

quadrants of the $u_{-}-u_{+}$plane admit a single-valued, one-to-one mapping, comparable to the Riemann problem classifications for the $\mathrm{KdV}$ and KdVB equations with convex flux (recall figure 3.4). It is the remaining quadrants where non-convexity introduces novel features. The origin of the non coinciding boundaries for the Riemann problem classifications of $\mathrm{mKdVB}$ and $\mathrm{mKdV}$ can be traced back to the asymmetry introduced by the Rankine-Hugoniot and Lax entropy conditions (4.4) for mKdVB and the symmetric causality and convexity conditions $(5.20)$ for $\mathrm{mKdV}$.

A direct mapping is possible when $0<u_{-}<u_{+}$or $u_{+}<u_{-}<0$ for any fixed $\mu \neq 0$. This implies that, as $\nu \rightarrow 0^{+}, \mathrm{S}^{ \pm} \rightarrow \mathrm{DSW}^{ \pm}$. Diffusive-dispersive Lax shocks map to DSWs in this regime. This provides an explanation for the similarity (within the regime considered) between the Lax entropy condition (4.2) and the DSW admissibility conditions (5.20), the new feature being the existence of two DSW speeds. The rarefactions are continuous and exhibit only weak discontinuities (i.e., in higher derivatives), hence their regularizations are the same to leading order. 


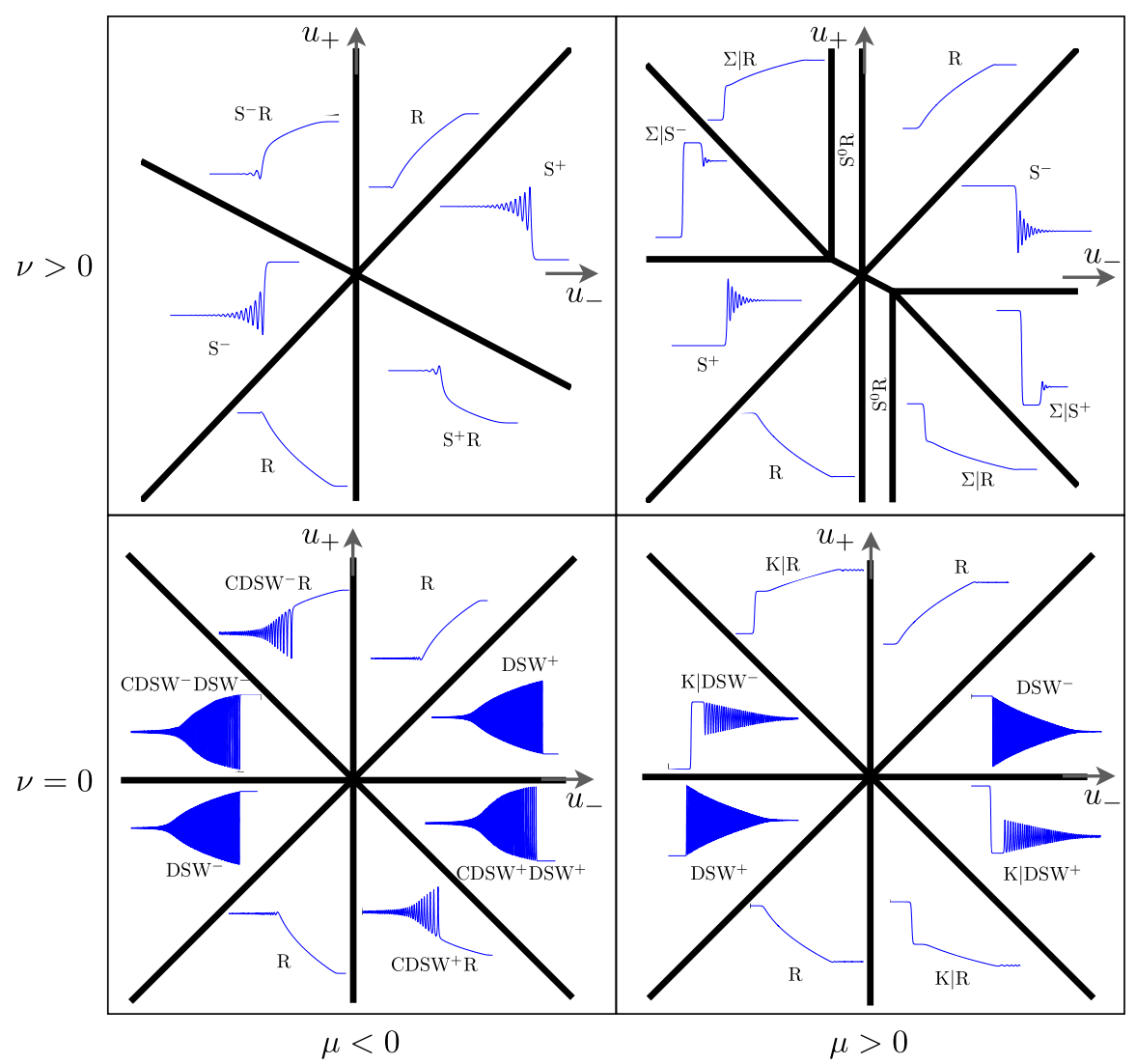

FIG. 6.6. Replicas of figures 4.2 and 5.3 with numerical solutions for interpretation of the zero diffusion mapping.

In the case of positive dispersion $\mu>0$, the diffusive-dispersive classification approaches the same octant structure as the dispersive classification in the zero diffusion limit because $\delta=\nu / \sqrt{\mu} \rightarrow 0^{+}$as $\nu \rightarrow 0^{+}$. This leads to the mappings $\Sigma\left|\mathrm{S}^{ \pm} \rightarrow \mathrm{K}\right| \mathrm{DSW}^{ \pm}$and $\Sigma|\mathrm{R} \rightarrow \mathrm{K}| \mathrm{R}$. Kinks are the purely dispersive analogue of diffusive-dispersive undercompressive shocks. This correspondence was similarly recognized in the context of two-layer fluids [59]. Numerical results in figure 6.1 show that the intermediate constant states associated with these double-wave structures attain different values according as $\nu>0$ or $\nu=0$. This is because a dispersive kink's profile always admits the symmetry $x \rightarrow-x, u \rightarrow-u$. An undercompressive shock is not symmetric and the relation between the two constant states that it connects changes with the parameter $\delta=\nu / \sqrt{\mu}$, as described in $\S 5.1 .1$. Nevertheless, the qualitative features between the diffusive-dispersive and dispersive solutions admit a natural correspondence. This correspondence with nonzero diffusion is neither oneto-one nor single-valued. There is the addition of the double-wave $\mathrm{S}^{0} \mathrm{R}$, which does not exhibit an intermediate constant state nor does the shock exhibit any oscillation. Complicating the mapping further is the fact that the boundaries between the different wave structures when $\nu>0$ and $\nu=0$ do not line up. Then the mapping allows for $\Sigma \mid \mathrm{R}$ or $\mathrm{S}^{0} \mathrm{R} \rightarrow \mathrm{K} \mid \mathrm{R}$ or $\mathrm{K} \mid \mathrm{DSW}{ }^{ \pm}$. 
The negative dispersion $\mu<0$ case is complicated by the fact that, for any nonzero diffusion, the Riemann problem classification divides the $u_{-}-u_{+}$plane into only six regions whereas the zero diffusion case involves eight. The new feature associated with zero diffusion is the existence of contact DSWs. As we have shown in previous sections, CDSWs have no "natural" diffusive-dispersive correlate. Nevertheless, the overlapping domains contained in the two octants $0<u_{-}<-u_{+},-u_{+}<u_{-}<0$ of figure 6.6 imply that $\mathrm{S}^{ \pm} \mathrm{R} \rightarrow \mathrm{CDSW}^{ \pm} \mathrm{R}$ as $\nu \rightarrow 0^{+}$in this region. Shock-rarefactions become CDSW-rarefactions in the zero diffusion limit. A careful examination of the numerical results shows that the constant state connecting these double wave structures differ for the diffusive and non-diffusive cases. This is a manifestation of the difference between the Rankine-Hugoniot relation and the CDSW conditions.

The zero diffusion mapping $\mathrm{S}^{ \pm} \rightarrow \mathrm{CDSW}^{ \pm} \mathrm{DSW}^{ \pm}$is implied by the regions $0<$ $u_{+}<-u_{-} / 2$ and $-u_{-} / 2<u_{+}<0$ in figure 6.6. Lax shocks map either to contact DSWs when attached to a rarefaction or to a hybrid CDSW-DSW or to a pure DSW, depending on the relation between $u_{-}$and $u_{+}$. This implies that the zero diffusion mappings of $\mathrm{S}^{ \pm}$and $\mathrm{S}^{ \pm} \mathrm{R}$ are multivalued. The top two panels in figure 6.1(a) show that, while there may be some subtle features introduced by CDSWs (recall figure 5.2 ), generally it is difficult to distinguish them from pure DSWs. Recall that CDSWs arise due to nonstrict hyperbolicity at the level of the Whitham modulation equations, a feature of the purely dispersive case. Therefore it is perhaps not surprising that diffusive-dispersive shocks (TWs) do not discern such features. The introduction of a non-convex flux implies that Lax shocks map to either CDSWs or DSWs but their differences may be difficult to identify in practice.

Aside from the ambiguity in the zero diffusion limit mapping of Lax shocks introduced by CDSWs when $\mu<0$, the other limits described so far are natural. However, the remaining, unexplored regions of the $u_{-}-u_{+}$plane with regards to the zero diffusion mapping are $u_{-} / 2<-u_{+}<u_{-}$and $u_{-}<-u_{+}<u_{-} / 2$ when $\mu<0$. Figure 6.6 shows $\mathrm{S}^{ \pm} \mathrm{R} \rightarrow \mathrm{CDSW}^{ \pm} \mathrm{DSW}^{ \pm}$in this region. But this mapping is peculiar because there is no rarefaction in the purely dispersive case. The resolution of this apparent contradiction can be understood by appealing to the small diffusion regime and relevant asymptotic time scales, which we undertake in the next subsection.

6.4. Time scales and critical scalings. Motivated by the singular transition $\mathrm{S}^{ \pm} \mathrm{R} \rightarrow \mathrm{CDSW}^{ \pm} \mathrm{DSW}^{ \pm}$just described, we now investigate the dynamics when the diffusion is small but nonzero. The existence of the parameter $\delta=\nu / \sqrt{|\mu|}$ in the TW solutions of $\S 4$ provides a clue as to the critical scaling relationship between diffusion and dispersion. By "small diffusion", we really mean $0<\nu \ll \sqrt{|\mu|}$. We now rescale space and time in (1.4) to demonstrate the time scales in which dispersion dominates the transient, intermediate asymptotic behavior, and the time scale on which the long-time behavior emerges, when diffusion and dispersion are in balance. In order to preserve the balance for transport on the left side of (1.4), we scale space and time by the same constant. Accordingly, let $t=a \tau, x=a y$. Then equation (1.4) transforms to

$$
u_{\tau}+\left(u^{3}\right)_{y}=\frac{\nu}{a} u_{y y}+\frac{\mu}{a^{2}} u_{y y y} .
$$

In order for the dispersive term to balance the transport term, we set $a=\sqrt{|\mu|}$. Then the diffusion coefficient of the first term on the right hand side becomes $\nu / \sqrt{|\mu|}$. If we take this ratio to be small, then the diffusive term is negligible compared to dispersion over the time interval where $t \sim \sqrt{|\mu|}$. 


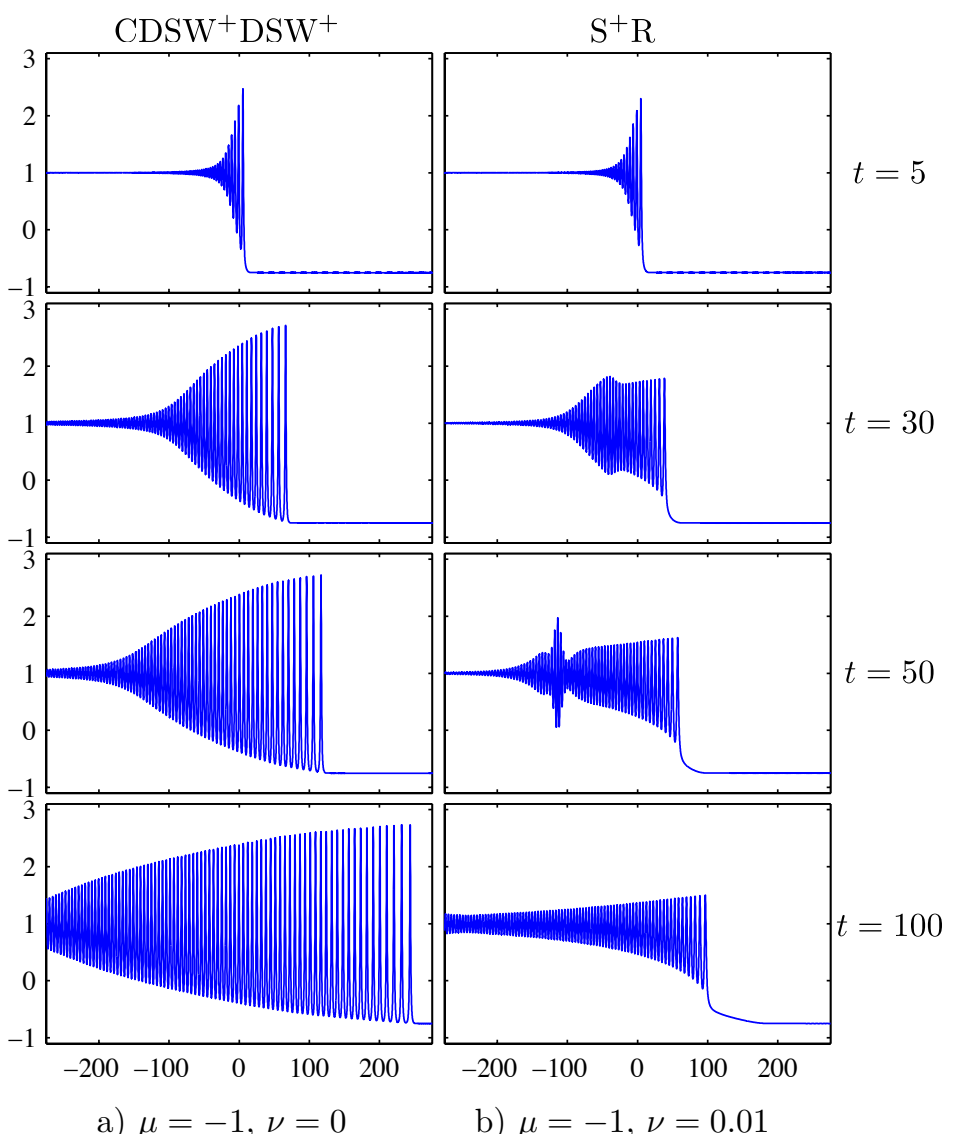

FIG. 6.7. Numerical simulation of the Riemann problem for $u_{-}=1, u_{+}=-0.75$ comparing purely dispersive (left) and diffusive-dispersive (right) transient dynamics.

Over long times, as the diffusive terms become significant, we expect solutions to converge to a combination of traveling waves and rarefactions, reflecting the combined effects of diffusion and dispersion. The time scale for such behavior emerges when the diffusive and dispersive terms are in balance. From (6.1) we see that the coefficients on the right hand side are equal when $a=|\mu| / \nu$. The corresponding time scale is $t \sim|\mu| / \nu$. Since $|\mu| / \nu=\sqrt{|\mu|}(\sqrt{|\mu|} / \nu) \gg \sqrt{|\mu|}$, we see that there is a transient regime $\sqrt{|\mu|} \ll t \ll|\mu| / \nu$ over which the solution evolves from dispersion-dominated to the diffusive-dispersive behavior analyzed here. This transient evolution resembles the diffusion-dominated classical theory except in cases where undercompressive waves appear.

As $\nu$ approaches zero with fixed $\mu$, the diffusive effects take longer to establish themselves, and in the limit $\nu \rightarrow 0^{+}$, the evolution is purely dispersive. The elongation of the transient dynamics time interval is suggestive of the transition between diffusivedispersive solutions of the Riemann problem and purely dispersive solutions. Figure 6.7 showcases this behavior for the numerical solution of a Riemann problem in the regime $u_{-} / 2<-u_{+}<u_{-}$and $\mu<0$ where apparently $\mathrm{S}^{+} \mathrm{R} \rightarrow \mathrm{CDSW}^{+} \mathrm{DSW}^{+}$as 
$\nu \rightarrow 0^{+}$. We fixed $\mu=-1$ and evolved the same initial data, a smoothed transition from $u_{-}=1$ to $u_{+}=-0.75$ for $\nu=0$ (figure $6.7(\mathrm{a})$ ) and $\nu=0.01$ (figure $6.7(\mathrm{~b})$ ). As predicted by the scaling analysis, the initial dynamics, e.g., $t=5=\mathcal{O}(\sqrt{|\mu|})$, for both the dispersive and diffusive-dispersive models are essentially the same. The models initiate the formation of DSWs. But in the transient regime $1=\sqrt{|\mu|} \ll t \ll$ $|\mu| / \nu=100$, we observe the dynamics corresponding to a transition from a DSW to a shock-rarefaction. A large wave packet propagates backward, through the initiated, approximate DSW, leaving a steady diffusive-dispersive shock configuration adjacent to a rarefaction in its wake. Also predicted by the scaling analysis, at $t=100=|\mu| / \nu$, the diffusive-dispersive behavior has set in for the solution profile. This example indicates that the resolution of the singular limit $\nu \rightarrow 0^{+}$involves nontrivial, transitory dynamics.

The transition $\mathrm{S}^{ \pm} \mathrm{R} \rightarrow \mathrm{CDSW}^{ \pm} \mathrm{DSW}^{ \pm}$is a clear example of the singular behavior of the zero diffusion limit, demonstrating how to properly interpret the zero diffusion mapping in table 6.1 as a dynamic process. First, nonlinearity alone approximately describes the dynamics up to wavebreaking. Then, dispersion leads to the generation of oscillations and finally diffusion and dispersion together resolve the solution into a TW. As noted in $\S 4.2$, the resulting TW can be interpreted as an underdamped oscillator. The damping of this oscillator goes to zero with $\delta$, hence approaches a continually expanding dispersive shock wave.

6.5. Small-diffusion regime and modulation equations. As we have seen, the analytical approaches used in the descriptions of diffusive-dispersive shocks and DSWs are quite different. The former is based on the analysis of an ODE for TW solutions of the mKdVB equation, while the latter employs nonlinear modulation theory, resulting in a system of homogeneous first order quasilinear PDEs. These two approaches can be reconciled in the case of small-diffusion $0<\nu \ll \sqrt{|\mu|}$. The reconciliation can be understood in the framework of an appropriate modification of Whitham modulation theory, in which the diffusive term in the mKdVB equation (1.4) is treated as a small perturbation of the $\mathrm{mKdV}$ equation. In this section, we briefly describe this approach for the well-studied case of the KdVB equation (3.4) and then outline its implications for the mKdVB case leaving a more careful analysis to future publications.

In 'perturbed' modulation theory, the balance laws of the perturbed equation (e.g., the KdVB equation) are averaged over a family of periodic solutions for the unperturbed equation (i.e., the KdV traveling wave solutions (3.7) [30]; see also [20]). The result of this procedure is a non-homogeneous modulation system

$$
\frac{\partial r_{j}}{\partial t}+V_{j}(\mathbf{r}) \frac{\partial r_{j}}{\partial x}=\delta R_{j}(\mathbf{r}), \quad j=1,2,3, \quad \delta=\frac{\nu}{\sqrt{|\mu|}} \ll 1,
$$

where $V_{j}(\mathbf{r})$ are the $\mathrm{KdV}$-Whitham characteristic speeds (3.10) and the right-hand sides $R_{j}(\mathbf{r})$ arise from averaging the diffusive terms in the perturbed KdV balance laws. See [4], [41] for the explicit expressions in terms of complete elliptic integrals. The modulation system (6.2) for the KdVB equation with small diffusion can also be obtained using a direct multiple scales procedure, equivalent to Whitham averaging (see [77]). An effective general method for the derivation of modulation systems for perturbed integrable equations was developed in [52].

Unlike the unperturbed modulation system (3.9), the perturbed system (6.2) possesses TW solutions $r_{j}(x-s t)$. It was shown in [4], [41] that the TW solution of the KdVB-Whitham system connects two constant, disparate states: $u \rightarrow u_{ \pm}$as $x \rightarrow \pm \infty$, 
$u_{-}>u_{+}$, and satisfies the Rankine-Hugoniot condition $s=\left(u_{-}+u_{+}\right) / 2$. Thus, this solution describes slow modulations of the classical diffusive-dispersive shock wave in the small diffusion regime. As a matter of fact, this outlined construction of the TW modulation solution is equivalent to a direct multiple scales perturbation analysis of the ODE (3.5) with $\delta \ll 1$ for the TW solutions of the KdVB equation itself (see [49]). However, modulation theory provides a much broader platform, enabling one to describe different stages of diffusive-dispersive shock development (see $[4,20]$ ).

The general method [52] for the derivation of perturbed modulation equations via spectral finite-gap theory, and its applications to the $\mathrm{mKdV}$ and Gardner equations in [56], [54], suggest that the perturbed modulation systems for mKdVB and KdVB are related by the same surjective mapping (5.9), (5.7) for $\mu<0$, and (5.10), (5.8) for $\mu>0$, as their unperturbed, purely dispersive, counterparts. This suggests that the modulation description of classical oscillating mKdVB shocks can be derived from known solutions of the KdVB modulation system. However, the description of oscillatory contact shocks in shock-rarefaction complexes for $\mu<0$ (see figure $6.7(\mathrm{~b}$ ), $t=100$ ) cannot be obtained from the KdVB modulation solutions and requires further analysis.

7. Conclusions. The analysis of Riemann problems for the $\operatorname{KdV}(\mathrm{B})$ and $\mathrm{mKdV}(\mathrm{B})$ models reviewed here provides an explicit link between the theories of hyperbolic conservation laws and nonlinear dispersive wavetrains. These are universal, asymptotic models of diffusive-dispersive Eulerian hydrodynamics. When the Eulerian pressure law is non-convex, the modified KdV-Burgers equation emerges. The prototypical phenomena arising from the competition between diffusion, dispersion, and non-convex flux include non-classical wave features such as undercompressive shock waves, and double wave complexes such as shock-rarefactions. The purpose of this work has been to compare the singular, zero diffusion limits of these waves with their purely dispersive counterparts, identifying notable similarities and differences.

An essential difference between diffusive-dispersive Lax shocks and classical dispersive shock waves is that the former have fixed, traveling wave profiles whereas the latter waves have expanding, oscillating profiles. Consonant to this difference are the dissimilar shock jump conditions and their derivation. Nevertheless, comparing $\mathrm{KdVB}$ to $\mathrm{KdV}$, there is a relatively simple, bijective zero diffusion mapping from Lax shocks and rarefactions with diffusion to DSWs and rarefactions without diffusion. The introduction of a non-convex flux in the mKdVB and mKdV equations leads to a more complex, multivalued mapping. Now, the sign of the dispersion term plays an essential role. For negative dispersion and nonzero diffusion, the classical theory of conservation laws prevails. The new feature resulting from a non-convex flux is the shock-rarefaction, not essentially reliant upon the dispersive term in the equation. In this regard, the small diffusion regime provides limited clues to the purely dispersive dynamics, where a new and unique feature arises, the contact DSW. These solutions occur due to the nonstrict hyperbolicity of the Whitham modulation equations. A CDSW is non-classical in the sense that either one characteristic family passes through it, as in a CDSW-DSW, or one characteristic family becomes tangential to the characteristics of an adjacent wave, as in a CDSW-rarefaction. The former behavior is analogous to an undercompressive shock, which also has characteristics passing through, but occurs only in the positive dispersion regime. This behavior of characteristics in CDSWs contrasts sharply with the fact that all diffusive-dispersive shocks in the negative dispersion regime satisfy the Lax entropy conditions. As a result, there is some ambiguity in the zero diffusion limit of Lax shocks. We explain and 
resolve the ambiguity for small diffusion by identifying relevant time scales that include a transient window during which the dispersive-dominated dynamics transition to a diffusive-dispersive balance.

For positive dispersion, the diffusive-dispersive dynamics are fundamentally different from the negative dispersion regime. Here, the role of dispersion is essential for the nonzero diffusion theory. This is due to the existence of non-classical TW solutions, namely undercompressive shocks, that are not available in the dispersionless regime. The zero diffusion mapping identifies an undercompressive shock with a kink or undercompressive DSW. Kinks are monotone traveling waves satisfying the Rankine-Hugoniot shock conditions, and consequently do not exhibit the expansionary and oscillatory structure associated with classical DSWs. The Lax entropy conditions are not fulfilled for kinks because all characteristics pass directly through, without any deflection.

This work emphasizes the universal scalar dynamics occurring in diffusive - dispersive modifications (2.1) of non-convex Eulerian systems. Physically interesting dispersive and dispersive-diffusive systems exhibiting complex structure of solutions due to non-convex fluxes arise in fluid dynamics, plasma physics, magnetohydrodynamics, nonlinear optics, Bose-Einstein condensates and magnetization dynamics. As examples of important bi-directional non-convex dispersive systems we mention Miyata-Choi-Camassa system for fully nonlinear internal waves [12], [26] and the cubic-quintic nonlinear Schrödinger equation arising in nonlinear optics and BoseEinstein condensate dynamics [14].

The universality of the mathematical description developed in this paper is due to the generality of the mKdVB equation (1.4), which is obtained via the well-established procedure of multiple scale expansions, and captures all essential properties of unidirectional, weakly nonlinear, long-wave diffusive-dispersive dynamics occurring in the neighborhood of the linear degeneracy point (2.9) for the background flow. While the $\mathrm{mKdVB}$ description of the diffusive-dispersive dynamics of classical and non-classical shocks is based on the explicit calculations of TWs [47], the construction of the zerodiffusion DSW theory employs subtle integrability properties of the mKdV equation which are manifested, in particular, in the availability of Riemann invariants for the associated system of Whitham modulation equations. The extension of the present work to the fully nonlinear, vector (bi-directional propagation) case described by full non-convex Eulerian systems (2.1) or systems of similar structure will be a major development of the existing theory.

We note that system (2.1) can be re-written using Lagrangian co-ordinates as a diffusive-dispersive modification of a general $p$-system (see, e.g., [15]). The theory of undercompressive shocks for non-convex $p$-systems was constructed in $[68,89]$. The counterpart zero-diffusion theory is not available at present and its development will require an extension of the non-integrable methods of [22], [44] to the non-convex flux case. The inclusion of the possibility of non-convexity in the linear dispersion relation will introduce additional complexity to the Riemann problem classifications and their comparisons for diffusive-dispersive and pure dispersive cases.

It is hoped that this work will inspire further examination and exploitation of the bridge between the well-established field of hyperbolic conservation laws and the growing field of dispersive hydrodynamics.

Appendix A. Traveling wave solutions of the mKdV equation.

We present the classification of the traveling wave solutions of the $\mathrm{mKdV}$ equation which are used in the construction of various DSWs in Section 5.1. These solutions are 
obtained by integrating the ODE (5.1) for two cases $\mu>0$ and $\mu<0$. In describing traveling wave solutions we refer to two basic configurations of the potential curve $Q(u)$ shown in figure 5.1 .

If $\mu>0$ (positive dispersion), then the oscillations occur in the interval $u_{2} \leq u \leq$ $u_{3}$, where $Q(u) \geq 0$ (see figure 5.1(a)), and the solution of (5.1) is expressed in terms of Jacobi elliptic functions as

$$
u=u_{2}+\frac{\left(u_{3}-u_{2}\right) \operatorname{cn}^{2}\left(\theta, m_{1}\right)}{1-\frac{u_{3}-u_{2}}{u_{4}-u_{2}} \operatorname{sn}^{2}\left(\theta, m_{1}\right)},
$$

where

$$
\theta=\sqrt{\left(u_{3}-u_{1}\right)\left(u_{4}-u_{2}\right)} \eta / 2, \quad \eta=\frac{x-U t}{\sqrt{2|\mu|}}
$$

and

$$
m_{1}=\frac{\left(u_{3}-u_{2}\right)\left(u_{4}-u_{1}\right)}{\left(u_{4}-u_{2}\right)\left(u_{3}-u_{1}\right)},
$$

is the modulus, $0 \leq m_{1} \leq 1$. The soliton limit $m_{1} \rightarrow 1$ can be achieved in one of two ways: when $u_{2} \rightarrow u_{1}$ or when $u_{3} \rightarrow u_{4}$.

When $u_{2} \rightarrow u_{1}$, we obtain the "bright" soliton of elevation with amplitude $a=$ $u_{3}-u_{1}$ propagating against a constant background $u=u_{1}$,

$$
u=u_{1}+\frac{u_{3}-u_{1}}{\cosh ^{2} \theta-\frac{u_{3}-u_{1}}{u_{4}-u_{1}} \sinh ^{2} \theta} .
$$

The soliton speed $U$ is found from (5.3), (5.2)

$$
U=\frac{1}{2}\left(3 u_{1}^{2}+3 u_{1} u_{4}+u_{4}^{2}\right) .
$$

Analogously, for $u_{3} \rightarrow u_{4}$ one obtains a "dark" soliton of depression

$$
u=u_{4}-\frac{u_{4}-u_{2}}{\cosh ^{2} \theta-\frac{u_{4}-u_{2}}{u_{4}-u_{1}} \sinh ^{2} \theta},
$$

having the amplitude $a=u_{4}-u_{2}$ and propagating against the constant background $u=u_{4}$ with the speed

$$
U=\frac{1}{2}\left(3 u_{4}^{2}+3 u_{1} u_{4}+u_{1}^{2}\right)
$$

Note that, due to invariance of the $\mathrm{mKdV}$ equation with respect to the reflection transformation $u \mapsto-u$ formulas (A.6), (A.7) can be obtained from (A.4), (A.5) by replacing

$$
u \rightarrow-u, \quad u_{i} \rightarrow-u_{5-i}, \quad i=1,2,3,4
$$

Thus the mKdV equation with fixed $\mu>0$ admits the existence of solitons of both polarities. This is sharply distinct from the KdV dynamics where polarity of admissible solitary waves is uniquely related to the dispersion sign (see §3.2). This property of the mKdV equation is due to the quartic nature of the potential curve $Q(u)$ and 
is ultimately related to non-convexity of the hydrodynamic flux of the dispersionless limit (1.13).

If both $u_{2} \rightarrow u_{1}$ and $u_{3} \rightarrow u_{4}$ then the polynomial $Q(u)$ in the right-hand side of (5.1) has two double roots, which implies that the solution assumes the form of a kink/antikink, which can be obtained directly by integration of (5.1) with $u_{1}=u_{2}$ and $u_{3}=u_{4}$. Noting that due to (5.2) the double roots satisfy $u_{1}+u_{4}=0$ and choosing the constant of integration so that $u=0$ at $\eta=0$ we obtain:

$$
u= \pm \frac{1}{2}\left(u_{4}-u_{1}\right) \tanh \left[\frac{1}{2}\left(u_{4}-u_{1}\right) \eta\right]
$$

The lower sign corresponds to the kink with $u \rightarrow u_{4}$ as $\eta \rightarrow-\infty$ and $u \rightarrow u_{1}$ at $\eta \rightarrow \infty$; the upper sign yields the "anti-kink" with $u \rightarrow u_{1}$ as $\eta \rightarrow-\infty$ and $u \rightarrow u_{4}$ at $\eta \rightarrow \infty$. The speed of kink propagation in both cases is found from (5.3) to be $U=u_{1}^{2}$, which agrees with the classical shock speed (4.3), where $u_{+}=u_{1}, u_{-}=u_{4}=-u_{1}$.

We also present the small-amplitude asymptotics of the solution (A.1) when $m_{1} \rightarrow$ $0\left(u_{2} \rightarrow u_{3}\right)$. In this limit, the cnoidal wave (A.1) asymptotically transforms into a linear harmonic wave

$$
\begin{aligned}
& u \cong u_{2}+\frac{1}{2}\left(u_{3}-u_{2}\right) \cos (k \eta), \quad\left(u_{3}-u_{2}\right) \ll u_{2}, \\
& k=\sqrt{\left(u_{2}-u_{1}\right)\left(u_{4}-u_{2}\right)}, \quad U=\frac{1}{2}\left(u_{1}^{2}+u_{4}^{2}\right) .
\end{aligned}
$$

Now we turn to the case of negative dispersion $\mu<0$. The potential curve configuration is shown in figure 5.1(b), and one can see that finite, real-valued motion can occur either in the interval $u_{1} \leq u \leq u_{2}$ or in $u_{3} \leq u \leq u_{4}$. We only consider here the second case; the results for the first one can be obtained by applying the transformation (A.8).

Let $u_{3} \leq u \leq u_{4}$. Integration of (5.1) yields

$$
u=u_{3}+\frac{\left(u_{4}-u_{3}\right) \operatorname{cn}^{2}\left(\theta, m_{2}\right)}{1+\frac{u_{4}-u_{3}}{u_{3}-u_{1}} \operatorname{sn}^{2}\left(\theta, m_{2}\right)},
$$

where $\theta$ is defined by (A.2) and the modulus (cf. (A.3))

$$
m_{2}=\frac{\left(u_{4}-u_{3}\right)\left(u_{2}-u_{1}\right)}{\left(u_{4}-u_{2}\right)\left(u_{3}-u_{1}\right)}
$$

In the soliton limit $u_{3} \rightarrow u_{2}\left(m_{2} \rightarrow 1\right)$, we get

$$
u=u_{2}+\frac{u_{4}-u_{2}}{\cosh ^{2} \theta+\frac{u_{4}-u_{2}}{u_{2}-u_{1}} \sinh ^{2} \theta} .
$$

This is a "bright", elevation soliton with amplitude $a=u_{4}-u_{2}$. Its propagation speed is found from $(5.3),(5.2)$ to be

$$
U=\frac{1}{2}\left(u_{4}^{2}+2 u_{2} u_{4}+3 u_{2}^{2}\right)
$$

The limit $m_{2} \rightarrow 0$ can be reached in two ways. 
(1) If $u_{3} \rightarrow u_{4}$, we get asymptotically

$$
\begin{aligned}
& u \cong u_{3}+\frac{1}{2}\left(u_{4}-u_{3}\right) \cos (k \eta), \quad u_{4}-u_{3} \ll u_{3}, \\
& k=\sqrt{\left(u_{3}-u_{1}\right)\left(u_{3}-u_{2}\right)}, \quad U=\frac{1}{2}\left(3 u_{4}^{2}+2 u_{2} u_{4}+u_{2}^{2}\right) .
\end{aligned}
$$

This is a small-amplitude harmonic limit analogous to (A.10) obtained for $\mu>0$.

(2) If $u_{2}=u_{1}$, but $u_{3} \neq u_{4}$ then we arrive at the nonlinear trigonometric solution

$$
u=u_{3}+\frac{u_{4}-u_{3}}{1+\frac{u_{4}-u_{1}}{u_{3}-u_{1}} \tan ^{2} \theta}
$$

where

$$
\theta=\sqrt{\left(u_{3}-u_{1}\right)\left(u_{4}-u_{1}\right)} \eta / 2, \quad U=\frac{1}{8}\left(3 u_{3}^{2}+3 u_{4}^{2}+2 u_{3} u_{4}\right),
$$

and the amplitude $a=u_{4}-u_{3}$ is generally order unity. The nonlinear trigonometric solution (A.13) has no analogs in the KdV theory. Indeed, the very existence of the nonlinear trigonometric solution is possible due to the quartic nature of the potential function $Q(u)$, i.e. due to the non-convex hyperbolic flux of the $\mathrm{mKdV}$ equation.

If $u_{3} \rightarrow u_{2}=u_{1}$, then $\theta \rightarrow 0$ and solution (A.13) transforms into an algebraic soliton of elevation,

$$
u=u_{1}+\frac{u_{4}-u_{1}}{1+\left(u_{4}-u_{1}\right)^{2} \eta^{2} / 4}, \quad U=3 u_{1}^{2} .
$$

Note that the algebraic soliton propagates with the characteristic speed of the hyperbolic limit (1.13) evaluated at the background $u=u_{1}$. The counterpart "dark" solutions corresponding to the interval $u_{1} \leq u \leq u_{2}$ are obtained from (A.11) - (A.14) by applying the reflection transformation (A.8).

Finally, we mention a family of periodic solutions corresponding to the potential curve configuration where the roots $u_{2}$ and $u_{3}$ are complex conjugates. Such solutions are modulationally unstable [18] but they do not arise in the Riemann problem so we do not describe these solutions here. See [25] for the proof of hyperbolicity of the zero dispersion limit of the $\mathrm{mKdV}$ equation with $\mu<0$.

\section{Appendix B. Numerical method.}

Here we describe the numerical method of solution for the $\operatorname{KdV}(\mathrm{B})$ and $\mathrm{mKdV}(\mathrm{B})$ equations. The stiffness of the third order derivative term, the long time integration required, and the large domain needed to properly resolve the wave solutions make this a challenging computational problem. A high order, integrating factor (IF), pseudospectral Fourier method that incorporates differing, constant boundary values is developed, motivated by Trefethen's approach to solving KdV [92]. The IF-RK4 method, utilizing the fourth-order Runge-Kutta algorithm (RK4), has been shown to perform only slightly slower than other state-of-the-art methods for the KdV and Burgers equations [57]. Its ease of formulation and implementation make IF-RK4 attractive.

Interest here is in the long time behavior of the Riemann Problem

$$
\begin{aligned}
& u_{t}+c_{0} u^{p} u_{x}=\nu u_{x x}+\mu u_{x x x}, \quad x \in \mathbb{R}, \quad t>0, \\
& u(x, 0)= \begin{cases}u_{-} & x<0, \\
u_{+} & x>0 .\end{cases}
\end{aligned}
$$


The parameters include $c_{0} \in\{1,3\}, p \in\{1,2\}, \nu \geq 0, \mu \in \mathbb{R}$.

We consider a truncated domain $x \in[-L, L]$ and assume that, to an appropriate level of approximation, $u( \pm L, t)=u_{ \pm}$and $\partial_{x} u(x, t)$ is smooth and compactly supported on $[-L, L]$ for each $t \in[0, T]$, some $T>0$. Then, the function $v(x, t)$ defined by

$$
v(x, t)=u_{x}(x, t), \quad u(x, t)=\int_{-L}^{x} v(y, t) \mathrm{d} y+u_{-},
$$

is smooth and compactly supported in this domain. Differentiating (B.1) gives a nonlocal equation for $v$

$$
v_{t}+c_{0}\left(u^{p} v\right)_{x}=\nu v_{x x}+\mu v_{x x x} .
$$

Since $v(x, t)$ is localized within $[-L, L]$, we can treat its periodic extension with the Fourier series expansion

$$
v(x, t)=\sum_{n} \widehat{v}_{n}(t) e^{i k_{n} x}, \quad \widehat{v}_{n}(t)=\frac{1}{2 L} \int_{-L}^{L} v(x, t) e^{-i k_{n} x}, \quad k_{n}=n \pi / L .
$$

The Fourier coefficients $\widehat{v}_{n}$ satisfy the differential equations

$$
\frac{\mathrm{d} \widehat{v}_{n}}{\mathrm{~d} t}+i \mu k_{n}^{3} \widehat{v}_{n}=-i c_{0} k_{n} \widehat{\left(u^{p} v\right)_{n}}-\nu k_{n}^{2} \widehat{v}_{n} .
$$

Letting

$$
\widehat{V}_{n}(t)=e^{i \mu k_{n}^{3} t} \widehat{v}_{n}(t)
$$

then

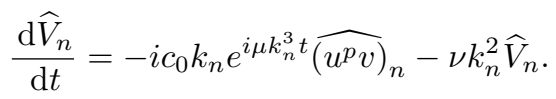

A suitable truncation of the Fourier series is chosen, $|n|<N$, and the corresponding eqs. (B.5) are then stepped forward in time by the standard RK4 method. The stiffest, third derivative term from (B.4) is absorbed into the transformation to $\widehat{V}_{n}$, thus allowing for stable timestepping with larger $\Delta t$. We do not incorporate the second derivative term into the phase transformation because it leads to exponentially large/small terms during the calculation and causes numerical instability.

A calculation shows that the accumulation function in (B.3) satisfies

$$
u(x, t)=\sum_{n \neq 0} \frac{\hat{v}_{n}(t)}{i k_{n}} e^{i k_{n} x}-\frac{1}{2 L} \int_{-L}^{L} x v(x, t) \mathrm{d} x+(x+L) \frac{u_{+}-u_{-}}{2 L}+u_{-} .
$$

Equation (B.6) is suitable for numerical computation and is used for the reconstruction of $u(x, t)$ where all Fourier series coefficients are computed using the FFT. The integral in (B.6) is computed with the trapezoidal rule. So long as the solution $v(x, t)$ remains localized within $[-L, L]$, this method is spectrally accurate.

The initial condition (B.2) is smoothed by a hyperbolic tangent profile with width one.

For the computations presented in this work, the numerical method was thoroughly tested and validated using known, exact traveling wave solutions. Generally, 
the purely dispersive computations required more care due to the radiation of small amplitude dispersive waves to the boundary. We monitored the conserved quantity $\int_{-L}^{L} v(x, t) \mathrm{d} x$ to be within $10^{-13}$ its nominal value $u_{+}-u_{-}$across all simulations. The deviation of $u$ at the boundaries from $u_{ \pm}$was maintained below $10^{-4}$ (usually well below that). The numerical parameters were informed by the validation studies and chosen based upon the particular Riemann problem considered. Typically, we used $\Delta x \in\{0.05,0.1,0.2\}, \Delta t \in\left\{10^{-3}, 10^{-4}, 5 \cdot 10^{-5}\right\}$, and $L \in\{400,800,1600\}$.

\section{REFERENCES}

[1] R. Abeyaratne and J.K. Knowles, Kinetic relations and the propagation of phase boundaries in solids. Arch. Rational Mech. Anal., 114 (1991), 119-154.

[2] M.J. Ablowitz, Nonlinear Dispersive Waves. Asymptotic Analysis and Solitons (Cambridge University Press, 2011).

[3] M. J. Ablowitz and D. E. Baldwin, Dispersive shock wave interactions and asymptotics, Phys. Rev. E, 87, 022906 (2013).

[4] V.V. Avilov, I.M. Krichever and S.P. Novikov, Evolution of Whitham zone in the theory of Korteweg-de Vries, Sov. Phys. Dokl. 32 564-566 (1987).

[5] T.B. Benjamin and M.J. Lighthill, On cnoidal waves and bores Proc. Roy. Soc., A 224 (1954) 448-460.

[6] S. Benzoni-Gavage, Planar traveling waves in capillary fluids, Differential and integral equations, Khayyam Publishing 26 (2013) 433-478.

[7] S. Benzoni-Gavage, P. Noble and L. M. Rodrigues, Slow modulations of periodic waves in Hamiltonian PDEs, with application to capillary fluids, Journ. Nonlin. Sci. 24 (2014) 711768.

[8] A.L. Bertozzi, A. Munch and M. Shearer, Undercompressive shocks in thin film flows. Physica D, 134 (1999), 431-464.

[9] J.L. Bona and M. E. Schonbeck, Travelling wave solutions to the Korteweg - de Vries-Burgers equation, Proc. Roy. Soc. Edinburgh, 101 A (1985) $207-226$.

[10] A. Bressan. Hyperbolic systems of conservation laws: the one-dimensional Cauchy problem, Mathematics and its Applications vol. 20. Oxford Univ. Press, 2000.

[11] G. Chanteur and M. Raadu, Formation of shocklike modified Korteweg-de Vries solitons: Application to double layers, Phys. Fluids 302708 (1987).

[12] W. Choi and R. Camassa, Fully nonlinear internal waves in a two-fluid system., J. Fluid Mech. 396 (1999) 1-36.

[13] D.R. Christie, Long nonlinear waves in the lower atmosphere, J. Atmos. Sci., 46, 1462 -1491 (1989).

[14] M. Crosta, S. Trillo, and A. Fratalocchi, Crossover dynamics of dispersive shocks in BoseEinstein condensates characterized by two- and three-body interactions, Phys. Rev. A 85 (2012) 043607.

[15] C.M. Dafermos, Hyperbolic Conservation Laws in Continuum Physics, Springer (2009) New York.

[16] P. Deift, S. Venakides, and X. Zhou, New results in small dispersion KdV by an extension of the steepest descent method for Riemann-Hilbert problems, Int. Math. Res. Notices, 6, 285-299 (1997).

[17] R.J. DiPerna, Decay and asymptotic behavior of solutions to nonlinear hyperbolic systems of conservation laws. Indiana Univ. Math. J. 24 (1974/75), no. 11, 1047-1071.

[18] C.F. Driscoll and T. M. O'Neil, Modulational instability of cnoidal wave solutions of the modified Kortweg-de Fries equation, J. Math. Phys. 17, 1196 (1975).

[19] B. A. Dubrovin, On Hamiltonian perturbations of hyperbolic systems of conservation laws, II: Universality of critical behavior, Comm. Math. Phys., 267, 117-139 (2006).

[20] B.A. Dubrovin and S.P. Novikov, Hydrodynamics of weakly deformed soliton lattices. Differential geometry and Hamiltonian theory, Russian Math. Surveys, 44 35-124 (1989).

[21] I. Egorova, Z. Gladka, V. Kotlyarov, G. Teschl, Long-time asymptotics for the Korteweg-de Vries equation with step-like initial data, Nonlinearity, 261839 -1864 (2013).

[22] G.A. El, Resolution of a shock in hyperbolic systems modified by weak dispersion, Chaos, 15 (2005) 037103

[23] G. A. El, R. H. J. Grimshaw, and M. V. Pavlov, Integrable shallow-water equations and undular bores, Stud. Appl. Math., 106, 157-186 (2001). 
[24] G.A. El and M.A. Hoefer, Dispersive shock waves and modulation theory, submitted to Physica D.

[25] N. M. Ercolani, S. Jin, C. D. Levermore, and W. D. MacEvoy, The zero-dispersion limit for the odd flows in the focusing Zakharov-Shabat hierarchy Int. Math. Res. Notices, 47, 2529 (2003).

[26] J.G. Esler and J. D. Pierce, Dispersive dam-break and lock-exchange flows in a two-layer fluid, J. Fluid Mech., 667, 555 (2011)

[27] L.C. Evans. Partial Differential Equations, vol. 19. Graduate Series in Mathematics, Amer. Math. Soc., 2010.

[28] H. Flaschka, M. G. Forest, and D. W. McLaughlin, Multiphase averaging and the inverse spectral solution of the Korteweg-de Vries equation, Comm. Pure Appl. Math. 33, 739$784(1980)$.

[29] M.G. Forest and J. Lee, Geometry and modulation theory for the periodic nonlinear Schrödinger equation, in Oscillation Theory, Computation, and Methods of Compensated Compactness, Ed. by C. Dafermos, J. L. Ericksen, D. Kinerlehrer, and M. Slemrod, IMA Volumes on Mathematics and Its Applications 2, 35-69, Springer, New York (1986).

[30] M.G. Forest and D.W. McLaughlin, Modulations of perturbed KdV wave trains, SIAM J. Appl. Math. 44, 278-300 (1984).

[31] J. Glimm, Solutions in the large for nonlinear hyperbolic systems of equations. Comm. Pure Appl. Math., 18 (1965), 697-715.

[32] J. Glimm and P.D. Lax, Decay of Solutions of Systems of Nonlinear Hyperbolic Conservation Laws. Memoirs Amer. Math. Soc. vol. 101, 1970.

[33] H. Grad and P.N. Hu, Unified shock profile in a plasma, Phys. Fluids 102596 (1967).

[34] T. Grava and C. Klein, Numerical solution of the small dispersion limit of Korteweg-de Vries and Whitham equations, Comm. Pure Appl. Math. 60, 1623 -1664 (2007).

[35] T. Grava and C. Klein, Numerical study of a multiscale expansion of the Korteweg-de Vries equation and Painlevé-II equation, Proc. Roy. Soc. A 464 733-757 (2008).

[36] T. Grava, V.U. Pierce and F.-R. Tian, Initial value problem of the Whitham equations for the Camassa-Holm equation, Physica D 238, 55-66 (2009).

[37] O. Guès, G. Métivier, M. Williams, and K. Zumbrun, Nonclassical multidimensional viscous and inviscid shocks, Duke Math. J. 142 (2008), 1-110.

[38] A.V. Gurevich, A.L. Krylov, and G.A. El, Nonlinear modulated waves in dispersive hydrodynamics, Sov. Phys. JETP, 71, 899-910 (1990).

[39] A.V. Gurevich, A.L. Krylov, and G.A. El, Breaking problem in dispersive hydrodynamics, JETP Lett., 54, 102-107 (1991).

[40] A.V. Gurevich and L.P. Pitaevskii, Nonstationary structure of a collisionless shock wave Sov. Phys. JETP 38291 (1974).

[41] A.V. Gurevich, and L.P. Pitaevskii, Averaged description of waves in the Korteweg-de VriesBurgers equation, Sov. Phys. JETP, 66490 (1987).

[42] B.T. Hayes and P.G. LeFloch, Nonclassical shocks and kinetic relations: Scalar conservation laws, Arch. Rational Mech. Anal., 139, 1-56 (1997).

[43] B. Hayes and M. Shearer, Undercompressive shocks and Riemann problems for scalar conservation laws with non-convex fluxes, Proc. Roy. Soc. Edinburgh A129, 733 - 754 (1999).

[44] M.A. Hoefer, Shock waves in dispersive Eulerian fluids, Journ. Nonlin.Sci. 24 (2014) 525-577.

[45] M. Hoefer and M. Ablowitz, Dispersive shock waves. Scholarpedia, 4(11) 5562 (2009).

[46] M.A. Hoefer, M.J. Ablowitz, I. Coddington, E.A. Cornell, P. Engels, and V. Schweikhard, Dispersive and classical shock waves in Bose-Einstein condensates and gas dynamics, Phys. Rev. A 74, 023623 (2006).

[47] D. Jacobs, B. McKinney, and M. Shearer, Traveling wave solutions of the modified Korteweg - de Vries-Burgers equation, Journ. Diff. Equations 116, 448 - 467 (1995).

[48] S. Jin, C. D. Levermore, and D. W. McLaughlin, The semiclassical limit of the defocusing NLS hierarchy, Comm. Pure Appl. Math., 52, 613-654 (1999).

[49] R.S. Johnson, A non-linear equation incorporating damping and dispersion, J. Fluid Mech., 42, 49-60 (1970).

[50] R.S. Johnson, A Modern Introduction to the Mathematical Theory of Water Waves (Cambridge University Press, 1997).

[51] A.M. Kamchatnov, Nonlinear Periodic Waves and Their Modulations-An Introductory Course, World Scientific (2000). Singapore.

[52] A.M. Kamchatnov, On Whitham theory for perturbed integrable equations, Physica D 188, $247-261$ (2004).

[53] A. M. Kamchatnov, Y. V. Kartashov,1 P.-É. Larré, and N. Pavloff, Nonlinear polarization waves in a two-component Bose-Einstein condensate, Phys. Rev. A 89, 033618 (2014) 
[54] A.M. Kamchatnov, Y.-H. Kuo, T.-C. Lin, T.-L. Horng, S.-C. Gou, R. Clift, R., G.A. El \& R.H.J. Grimshaw, Undular bore theory for the Gardner equation, Phys. Rev. E 86 (2012) 036605.

[55] A. M. Kamchatnov, Y.-H. Kuo, T.-C. Lin, T.-L. Horng, S.-C. Gou, R. Clift, R., G. A. El \& R. H. J. Grimshaw, Transcritical flow of a stratified fluid over topography: analysis of the forced Gardner equation, Journ. Fluid Mech. 736, 495 - 531 (2013).

[56] A.M. Kamchatnov, A. Spire, V.V. Konotop, On dissipationless shock waves in a discrete nonlinear Schrödinger equation, J. Phys. A: Math. Gen. 37, 5547 (2004).

[57] A.-K. Kassam and L.N. Trefethen, Fourth-Order Time-Stepping for Stiff PDEs, SIAM J. Sci. Comput. 26, 1214-1233 (2005).

[58] I.M. Krichever, Method of averaging for two-dimensional "integrable" equations, Func. Anal. Appl. 22, 200 - 2013 (1988).

[59] A. Kluwick, S. Scheichl, and E.A. Cox, Near-critical hydraulic flows in two-layer fluids, J. Fluid Mech. 575187 (2007).

[60] S.N. Kruzhkov. First order quasilinear equations in several independent variables. Math. USSR Sb., 10(2):217-243, 1970.

[61] Y. Kodama, V.U. Pierce and F.-R. Tian, On the Whitham equations for the defocusing complex modified KdV equation, SIAM J. Math. Anal. 401750 (2008).

[62] V.R. Kudashev, "Wave-number conservation" and succession of symmetries during a Whitham averaging, JETP Lett. 54 (1991) 175 - 178

[63] P. D. Lax. Hyperbolic systems of conservation laws II. Comm. Pure Appl. Math., 10 (1957), $537-566$.

[64] P.D. Lax, Hyperbolic Systems of Conservation Laws and the Mathematical Theory of Shock Waves. Conference Board of the Mathematical Sciences, Regional Conference Series in Applied Mathematics (SIAM, Philadelphia, 1973).

[65] P.D. Lax \& C.D. Levermore, The small dispersion limit of the Korteweg - de Vries equation I, II, III. Comm. Pure Appl. Math. 36 (1983) 253-290, 571-593, 809-829.

[66] J.A. Leach, An initial-value problem for the defocusing modified Korteweg - de Vries equation, Journ. Diff. Eq. 2521032 (2012).

[67] J.A. Leach and D.J. Needham, The large-time development of the solution to an initial-value problem for the Korteweg de Vries equation: I. Initial data has a discontinuous expansive step, Nonlinearity, 21, 2391-2408 (2008).

[68] P.G. LeFloch, Hyperbolic systems of conservation laws (Birkhauser, 2002)

[69] P.G. LeFloch and M. Shearer, Nonclassical Riemann solvers with nucleation, Proc. Roy Soc Edinburgh., 134A, 961-984 (2004).

[70] P.G. LeFloch and S. Mishra, Numerical methods with controlled dissipation for small-scale dependent shocks, Acta Numerica, 23, 743-816 (2014).

[71] C.D. Levermore, The hyperbolic nature of the zero dispersion KdV limit. Comm. Partial Differential Equations 13, 495-514 (1988).

[72] N.K. Lowman and M.A. Hoefer, Dispersive shock waves in viscously deformable media, Journ. Fluid Mech. 718, 524-557 (2013).

[73] A.J. Majda The Existence of Multi-dimensional Shock Fronts. Memoirs Amer. Math. Soc. vol. 281,1983

[74] A.J. Majda The Stability of Multi-dimensional Shock Fronts. Memoirs Amer. Math. Soc. vol. $275,1982$.

[75] T.R. Marchant, Undular bores and the initial-boundary value problem for the modified Korteweg-de Vries equation, Wave Motion, 45, 540 (2008).

[76] R.M. Miura, Korteweg - de Vries equation and generalizations. I. A remarkable explicit nonlinear transformation, J. Math. Phys. 91202 - 1204 (1968).

[77] S. Myint and R.H.J. Grimshaw, The modulation of nonlinear periodic wavetrains by dissipative terms in the Korteweg - de Vries equation, Wave Motion, 22, 215-238 (1995).

[78] S.P. Novikov, S.V. Manakov, L.P. Pitaevskii, and V.E. Zakharov, The Theory of Solitons: The Inverse Scattering Method. Consultants (1984) New York.

[79] O. Oleinik, On the uniqueness of the generalized solution of the Cauchy problem for a nonlinear system of equations occurring in mechanics, (in Rus- sian), Usp. Mat. Nauk (N.S.) 12 (1957)169176.

[80] O. Oleinik, Discontinuous solutions of nonlinear differential equations, Amer. Math. Soc. Transl. Ser. 26 (1963) 95172.

[81] M. V. Pavlov, Nonlinear Schrödinger equation and the Bogolyubov-Whitham method of averaging, Teor. Mat. Fiz., 71, 351-356 (1987).

[82] M.V. Pavlov, Double Lagrangian representation of $\mathrm{KdV}$ and the general solution of the Whitham equations, Russian Acad. Sci. Dokl. Math. 50, 400 (1995). 
[83] V.U. Pierce and F.-R. Tian, Self-similar solutions of the non-strictly hyperbolic Whitham equations, Comm. Math. Sci. 4799 (2006)

[84] J.E. Rothenberg and D. Grischkowsky, Observation of the formation of an optical intensity shock and wave breaking in the nonlinear propagation of pulses in optical fibers, Phys. Rev. Lett., 62, 531-534 (1989).

[85] M. Ruderman, T. Talipova, E. Pelinovskii, Dynamics of modulationally unstable ion-acoustic wavepackets in plasmas with negative ions, J. Plasma Physics, 74 (2008) 639-656

[86] R.Z. Sagdeev, The fine structure of a shock-wave front propagated across a magnetic field in a rarefied plasma, Sov. Phys. Tech. Phys. 6867 (1962).

[87] R.Z. Sagdeev, Cooperative phenomena and shock waves in collisionless plasmas, in Reviews of Plasma Physics, ed. M.A. Leontovich Consultants Bureau, New York 423 (1966)

[88] D.G. Schaeffer and M. Shearer, Riemann problems for nonstrictly hyperbolic $2 \times 2$ systems of conservation laws. Trans. Amer. Math. Soc. 304 (1987), 267-306.

[89] M.R. Schulze and M. Shearer, Undercompressive shocks for a system of hyperbolic conservation laws with cubic nonlinearity, Journ. Math. Anal. Appl. 229, 344 - 362 (1999)

[90] N.F. Smyth and P. E. Holloway, Hydraulic jump and undular bore formation on a shelf break, J. Phys. Oceanog., 18, 947-962 (1988)

[91] K. Spayd and M. Shearer, The Buckley-Leverett equation with dynamic capillary pressure. SIAM J. Appl. Math., 71 1088-1108 (2011).

92] L.N. Trefethen, Spectral Methods in Matlab, SIAM, Philadelphia (2000).

[93] F.-R. Tian, Oscillations of the zero dispersion limit of the Korteweg-de Vries equation, Comm. Pure Appl. Math., 46, 1093-1129 (1993).

[94] S. Venakides, The zero-dispersion limit of the Korteweg-de Vries equation with non-trivial reflection coefficient, Comm. Pure Appl. Math., 38, 125-155 (1985).

[95] W. Wan, S. Jia, and J. W. Fleischer, Dispersive superfluid-like shock waves in nonlinear optics, Nat. Phys., 3, 46-51 (2007).

[96] S. Watanabe, Ion acoustic soliton in plasma with negative ion. J. Phys. Soc. Japan 53, 950 956 (1984).

[97] G.B. Whitham, Non-linear dispersive waves, Proc. Roy. Soc., A283, 238-291 (1965).

[98] G.B. Whitham, Linear and Nonlinear Waves, Wiley-Interscience, New York (1974).

[99] C.C. Wu, Magnetohydrodynamic Riemann problem and the structure of the magnetic reconnection layer, J. of Geophys. Res.: Space Physics, 100, 5579-5598 (1995). 